\title{
BIFURCATION OF CRITICAL PERIODS FOR PLANE VECTOR FIELDS
}

\author{
CARMEN CHICONE AND MARC JACOBS
}

\begin{abstract}
A bifurcation problem in families of plane analytic vector fields which have a nondegenerate center at the origin for all values of a parameter $\lambda \in \mathbf{R}^{N}$ is studied. In particular, for such a family, the period function $(\xi, \lambda) \mapsto P(\xi, \lambda)$ is defined; it assigns the minimum period to each member of the continuous band of periodic orbits (parametrized by $\xi \in \mathbf{R}$ ) surrounding the origin. The bifurcation problem is to determine the critical points of this function near the center with $\lambda$ as bifurcation parameter.

Generally, if the function $\rho$, given by $\xi \mapsto P\left(\xi, \lambda_{*}\right)-P\left(0, \lambda_{*}\right)$, vanishes to order $2 k$ at the origin, then it is shown that the period function, after a perturbation of $\lambda_{*}$, has at most $k$ critical points near the origin. If $\rho$ vanishes to infinite order, i.e., the center is isochronous, it is shown that the number of critical points of $P$ for perturbations of $\lambda_{*}$ depends on the number of generators of the ideal of all Taylor coefficients of $\rho(\xi, \lambda)$, where the coefficients are considered elements of the ring of convergent power series in $\lambda$. Specifically, if the ideal is generated by the first $2 k$ Taylor coefficients, then a perturbation of $\lambda_{*}$ produces at most $k$ critical points of $P$ near the origin. These theorems are applied to the quadratic systems with Bautin centers and to one degree of freedom "kinetic+potential" Hamiltonian systems with polynomial potentials. For the quadratic systems a complete solution of the bifurcation problem is obtained. For the Hamiltonian systems a number of results are proved independent of the degree of the potential and a complete solution is obtained for potentials of degree less than seven.
\end{abstract}

Aside from their intrinsic interest, monotonicity properties of the period function are important in the question of existence and uniqueness of autonomous boundary value problems, in the study of subharmonic bifurcation of periodic oscillations, and in the analysis of the problem of linearization. In this regard it is shown that a Hamiltonian system with a polynomial potential of degree larger than two cannot be linearized. However, while these topics are the subject of a large literature, the spirit of this paper is more akin to that of $\mathrm{N}$. Bautin's treatment of the multiple Hopf bifurcation for quadratic systems and the work on various forms of the weakened Hilbert's 16th problem first posed by V. Arnold.

Received by the editors October 18,1987 . Selected portions of this paper were presented at the SIAM Annual Meeting in Minneapolis, July, 1988.

1980 Mathematics Subject Classification (1985 Revision). Primary 58F14, 58F22, 58F30, 34C15.

Key words and phrases. Period function, center, bifurcation, quadratic system, Hamiltonian system, linearization.

This research was partially supported by the Air Force Office of Scientific Research under grant AF-AFOSR-89-0078. 


\section{INTRODUCTION}

In this paper we consider analytic plane vector fields $(x, y) \mapsto \mathbf{X}(x, y, \lambda)$ which have a nondegenerate center at the origin for all values of the parameter $\lambda \in \mathbb{R}^{N}$, and which, in addition, have $(x, y) \mapsto(-y, x)$ as their linearization at the origin. We choose an open interval on the $x$-axis containing the origin which is small enough so that any orbit passing through a nonzero point of this interval is periodic and includes the origin in its interior. For a point $\xi$ in this interval, we define $P(\xi, \lambda)$ to be the minimum period of the periodic trajectory through $(\xi, 0)$. The purpose of this paper is to study some aspects of the bifurcation of critical points of the period function $\xi \mapsto P(\xi, \lambda)$. That is, if $F(\xi, \lambda):=P_{\xi}(\xi, \lambda)$, then we study the solutions of the equation $F(\xi, \lambda)=$ 0 , near $\xi=0$, as the parameter $\lambda$ varies. In the course of this work we have developed some methods which are applicable to an abstract bifurcation equation $F(\xi, \lambda)=0$ when the function $F$ is analytic. These methods should prove useful in the analysis of a wide class of bifurcation problems.

In this abstract framework, we start with an analytic function $(\xi, \lambda) \mapsto$ $F(\xi, \lambda)$ and write its series, near $\xi=0$, as

$$
F(\xi, \lambda)=a_{0}(\lambda)+a_{1}(\lambda) \xi+a_{2}(\lambda) \xi^{2}+\cdots,
$$

where each function $\lambda \mapsto a_{k}(\lambda)$ is analytic, and where for each $\lambda$ the series is convergent in some neighborhood of $\xi=0$. Then, given a point $\lambda_{*}$ where $F\left(0, \lambda_{*}\right)=0$, we wish to know how many zeros of the function $\xi \mapsto F(\xi, \lambda)$ are near $\xi=0$ for perturbations $\lambda$ of $\lambda_{*}$. The analysis of this question falls naturally into two cases. First, it may happen that

$$
a_{0}\left(\lambda_{*}\right)=a_{1}\left(\lambda_{*}\right)=a_{2}\left(\lambda_{*}\right)=\cdots=a_{n}\left(\lambda_{*}\right)=0 \text { and } a_{n+1}\left(\lambda_{*}\right) \neq 0
$$

In this case we say the bifurcation point $\lambda_{*}$ has finite order $n$ and one can show that at most $n$ zeros bifurcate from $\left(0, \lambda_{*}\right)$. This result follows from elementary considerations and is quite easy to prove. The second case which arises is bifurcation from a point $\lambda_{*}$ of infinite order, i.e., $a_{k}\left(\lambda_{*}\right)=0$ for $k \geq 0$. Here, the analysis is more subtle and much less is known. The difficulty is that in order to analyze the bifurcations from a point of infinite order one must have some knowledge of all the coefficients $a_{k}$. This is one of the main problems which we address in this paper.

Consider a bifurcation point of infinite order and for simplicity assume this point is $\lambda=0$. If the coefficients $a_{k}$ are elements of the polynomial ring $\mathbf{R}\left[\lambda_{1}, \lambda_{2}, \ldots, \lambda_{N}\right]$, or for that matter the ring $\mathbf{R}\left\{\lambda_{1}, \lambda_{2}, \ldots, \lambda_{N}\right\}$, of convergent power series at 0 , both of which are Noetherian $[8,24]$, then the ideal $\left(a_{0}, a_{1}, \ldots\right)$ of all Taylor coefficients is generated by some initial segment $a_{0}, a_{1}, \ldots, a_{K}$ of these coefficients. Following N. Bautin [5], the functions $a_{i}$ for $i>K$ are written in terms of the functions in this initial segment, i.e.,

$$
a_{i}=\alpha_{i 1} a_{0}+\alpha_{i 2} a_{1}+\cdots+\alpha_{i K} a_{K},
$$


and then formally one has

$$
F(\xi, \lambda)=\sum_{i=0}^{K} a_{i}(\lambda) \xi^{i}\left(1+\Psi_{i}(\xi, \lambda)\right)
$$

where $\Psi_{i}(0, \lambda)=0$ for $i=0,1,2, \ldots, K$. Thus, the bifurcation function behaves like a polynomial of degree $K$ near $\lambda=0$. In particular, there will be a bifurcation to at most $K$ zeros near $\xi=0$ for values of $\lambda$ near $\lambda=0$. We will make this formal argument precise in $\S 2$. Then the problem of bifurcation from a zero of infinite order is reduced to obtaining the smallest value $K$ such that the corresponding initial segment is a basis for the ideal of all Taylor coefficients.

In this generality there is little hope of success. Nonetheless, in many practical situations arising in dynamical systems the sequence $a_{k}$ can be generated by natural recursive relationships where there is a reasonable expectation that the finite basis can be found. This is the problem solved by Bautin when the $a_{k}$, $k \geq 0$, are the coefficients of the return map on a section emanating from a weak focus.

For the bifurcation of critical points of the period function one must find the bifurcation points in the parameter space, and determine their orders. The location and analysis of the finite order bifurcation points is relatively uncomplicated. However, even the location of the bifurcation points of infinite order, i.e., the isochrones, is a challenging problem. In fact, the location of the isochrones is analogous to the Poincare center problem. Both problems are completely solved only in the case where the vector field is quadratic. However, once the isochrones have been located we compute the first few coefficients of the power series representation of $P_{\xi}$ in powers of $\xi$. In many problems these coefficients turn out to be polynomials in $R\left[\lambda_{1}, \lambda_{2}, \ldots, \lambda_{N}\right]$. When this occurs, these coefficients are tested successively to see if an element of the sequence is already in the ideal generated by its predecessors. For this process we make use of some mathematical algorithms for checking ideal membership which rely on the computation of a Gröbner basis for the ideal [10]. These algorithms are now widely available in various computer algebra systems such as REDUCE and MACSYMA. As soon as a candidate initial segment of generators for the ideal is found, we try to determine sufficient conditions for a polynomial to be in the ideal generated by this initial segment. Then, using the structure of the differential equation and the period function we try to show these conditions are met for all succeeding Taylor coefficients. Often the conditions for ideal membership are expressed as algebraic identities in some of the derivatives of the polynomial being tested for membership. Such conditions are then verified using the variational equations of the original differential equation. In other cases algebraic conditions for ideal membership are verified from an analysis of the recursion scheme used to generate the Taylor coefficients. Thus, in principle we can determine the finite initial segment of coefficients which generate 
the ideal of all Taylor coefficients of $F$ and, in turn, determine the bifurcation structure of its zeros. Of course, in practice, any one of the steps of the procedure just outlined can be very difficult to carry through.

In this paper, we analyze two fundamental cases: centers of quadratic systems $(\S 3)$ and centers of Hamiltonian systems which arise from Hamiltonians given in the form "kinetic+potential" where the potential is a polynomial (§4). We obtain a complete description of the bifurcation of critical points of the period function near the origin of all centers for the case of quadratic systems. In particular, we show that at most two critical points of the period function bifurcate from a "weak center" of any quadratic system and that there are quadratic systems with two critical points. Actually we prove much more. We are able to specify in detail the bifurcations which can occur in the various subvarieties of the full parameter space of quadratic systems which correspond to the centers. In the case of polynomial potentials we prove that the only potential (up to a constant multiple) with an isochronous center at the origin is $V(u)=\frac{1}{2} u^{2}$. We determine the complete bifurcation structure for the critical points of the period functions for all polynomial potentials of degree six or less and we are also able to show that for an even polynomial potential function of degree $N=2 n$, there are at most $n-2$ critical points which bifurcate from the origin, and there are perturbations which produce $k$ critical points near the origin for any $k \leq n-2$.

It is natural to compare our methods with a normal form approach. As is well known [40], there is an analytic coordinate transformation which transforms a planar analytic system of differential equations with a nondegenerate center at the origin to the normal form

$$
\dot{x}=-f\left(x^{2}+y^{2}\right) y, \quad \dot{y}=f\left(x^{2}+y^{2}\right) x .
$$

Once this is done, a further change to polar coordinates gives

$$
\dot{r}=0, \quad \dot{\theta}=f\left(r^{2}\right),
$$

and it follows that the period function is given by

$$
P(\xi)=2 \pi / f\left(\xi^{2}\right) .
$$

Thus, the period function and the scale function $f$ carry the same information. In particular, a plane analytic vector field with a nondegenerate center at the origin can be transformed to the system $\dot{x}=-y, \dot{y}=x$ with an analytic change of coordinates if and only if the center is isochronous. This observation and Theorem 4.1 imply that a Hamiltonian system with a polynomial potential of degree $N>2$ which has a nondegenerate center at the origin cannot be linearized by an analytic change of coordinates. Moreover, it also turns out that the number of critical points of the period function which bifurcate near a center of finite order is an analytic invariant of the vector field. This number is reflected in the normal form of the vector field in the sense of A. Baider and R. Churchill [4]. They show that a formal change of coordinates will transform 
a plane vector field with a center at the origin to the form $\dot{r}=0, \dot{\theta}=1$ or the form $\dot{r}=0, \dot{\theta}=1 \pm r^{2 k}$. The first form corresponds to the isochronous case, the second to a vector field with a weak center of order $k$, where $k$ is an analytic invariant.

One could, for example, use the normal form in [40, §27] to obtain our results on the finite order bifurcations of the critical points of the period function, since this only requires the calculation of a finite number of terms of the normal form. We have not used this approach because our analysis of the bifurcations of critical points of the period function near an isochronous center requires us to determine the ideal of all Taylor coefficients of $P_{\xi}$. The corresponding problem using normal forms involves the ideal of all Taylor coefficients of the derivative of the scale function $f$. This is a very difficult problem. Indeed, an analogous ideal membership problem was raised by Siegel and Moser [40, p. 203] after their normal form calculation for the center-focus problem, and their question is still unsolved. However, it is important to note that the centerfocus problem really only requires knowledge of the variety of an ideal, and is thus analogous to Loud's results [27] on isochrones, whereas the complete solution of the more difficult problem on the bifurcation of limit cycles from a weak focus given by Bautin [5] requires a solution of the Siegel-Moser ideal membership problem for quadratic systems. While it is true that Bautin's results on finite order bifurcations can be obtained from a normal form calculation, it should be noted that a normal form approach does not seem to be useful for the solution of the most difficult part of Bautin's theorem, namely, that at most three limit cycles bifurcate in the space of quadratic systems from a quadratic center, which is an infinite order bifurcation problem. Likewise, in our work, the main difficulties involve solving an infinite order bifurcation problem-the bifurcation of critical periods from an isochrone. In our approach we take advantage of the form of the original vector field (it is a low order polynomial vector field) and computations based on its variational equations to solve the relevant ideal membership problem. To obtain the same results from the normal form seems to require either a knowledge of the convergent transformation of coordinates which transforms the vector field to the normal form, allowing us to translate our variational argument to the transformed vector field, or an argument using induction which shows how the high order coefficients of the normal form are in the ideal generated by the first few low order coefficients. Neither option seems at present to lead to a simplification. Because it may be of some independent interest, the period coefficients for the normal form associated with Loud's system, which can be used to characterize the isochrones of quadratic systems, are discussed in Appendix C.

The history of the work on period functions goes back at least to 1673 when C. Huygens observed that the pendulum clock has a monotone period function and therefore oscillates with a shorter period when the energy is decreased, i.e., 
as the clock spring unwinds. He hoped to design a clock with isochronous oscillations in order to have a more accurate clock to be used in the navigation of ships. His solution, the cycloidal pendulum, is perhaps the first example of a nonlinear isochrone. The characterization of the vector fields with isochrones is still a difficult and unsolved problem. However, in addition to the identification of the isochronous centers of polynomial "kinetic+potential" Hamiltonians given in this paper, characterizations have also been obtained for general "kinetic+potential" Hamiltonians [45, 46], for quadratics [27], and for the Sibirskii centers [39]. Results of this type are, of course, crucial for our work. There are now a number of authors who deal with various questions related to the period function. Most of this work has been motivated by a desire to find sufficient conditions for the period function to be monotone $[11,15,16,37,41]$, since monotonicity is a nondegeneracy condition for the bifurcation of subharmonic solutions of periodically forced Hamiltonian systems [14, Chapter 11], and since monotonicity implies existence and uniqueness for certain boundary value problems $[6,12,43]$.

Most of the work on plane polynomial vector fields, including this paper, is related to the questions surrounding Hilbert's 16 th problem (cf. [7, 19, 20, $21,23,44,52]$, and their bibliographies) and its various weakened versions, i.e., problems which ask for the number of occurrences of some property of a system given by polynomials in terms of the degrees of the defining polynomials. Here we ask for the number of critical points of the period function which bifurcate from the origin for a polynomial vector field in the plane. This is a special case of the problem considered in [15]. These questions are similar to the more general problem of finding the number of zeros of Abelian integrals over polynomial Hamiltonians [3, p. 303; 33, 34, 47].

During the preparation of this paper we have been extremely fortunate to be able to discuss our work with a number of helpful people. We wish to thank especially Professors Richard Cushman, Ira Papick, Robert Roussarie, and Wolmer Vasconcelos for their valuable help.

\section{LOCAL BIFURCATION AT A CENTER}

In this section we study the local bifurcations of critical points of the period function at the origin. We always use the word center to denote a stationary point (taken to be $(0,0)$ ) for a planar system of differential equations, surrounded by a continuous family of periodic trajectories with the system of differential equations normalized so that its linearization at the center has eigenvalues $\pm i$.

We will refer several times to the results of Bautin [5]. In particular, any quadratic system with a periodic trajectory surrounding the origin can, by a linear change of coordinates, be expressed in Bautin's form:

$$
\begin{aligned}
& \dot{x}=\lambda_{1} x-y-\lambda_{3} x^{2}+\left(2 \lambda_{2}+\lambda_{5}\right) x y+\lambda_{6} y^{2}, \\
& \dot{y}=x+\lambda_{1} y+\lambda_{2} x^{2}+\left(2 \lambda_{3}+\lambda_{4}\right) x y-\lambda_{2} y^{2} .
\end{aligned}
$$


Since we are interested in centers, we always assume $\lambda_{1}=0$, and let the resulting system be denoted by $\mathbf{B}_{\lambda}, \lambda=\left(\lambda_{2}, \ldots, \lambda_{6}\right)$. In addition, we note that the Lyapunov or focal values are constant positive multiples of $v_{3}, v_{5}$, and $v_{7}$ where

$$
\begin{aligned}
& v_{3}(\lambda):=-\lambda_{5}\left(\lambda_{3}-\lambda_{6}\right), \\
& v_{5}(\lambda):=\lambda_{2} \lambda_{4}\left(\lambda_{3}-\lambda_{6}\right)\left(\lambda_{4}+5 \lambda_{3}-5 \lambda_{6}\right), \\
& v_{7}(\lambda):=-\lambda_{2} \lambda_{4}\left(\lambda_{3}-\lambda_{6}\right)^{2}\left(\lambda_{3} \lambda_{6}-2 \lambda_{6}^{2}-\lambda_{2}^{2}\right),
\end{aligned}
$$

and the system $\mathbf{B}_{\lambda}$ has a center at the origin if and only if $v_{3}(\lambda)=v_{5}(\lambda)=$ $v_{7}(\lambda)=0$. This is a version of the Center Theorem for quadratic systems.

Our results can be viewed as an analogue of the work on the bifurcation of limit cycles from a weak focus, especially the study of bifurcation of limit cycles from a weak focus of a quadratic system carried out by Bautin. This theory is the subject of a vast literature; we suggest $[1,5,19,52]$ for the reader unfamiliar with this topic. In Bautin's treatment of this bifurcation problem one considers a vector field with a stationary point at the origin with linearization of the form $\left(\lambda_{1} x-y, x+\lambda_{1} y\right)$. The return map $h$ is defined on an interval $[0, L)$ of the $x$-axis and periodic trajectories correspond to zeros of the succession function $d(\xi)=h(\xi)-\xi$, where $\xi$ is the distance coordinate from the origin along the positive $x$-axis. A focus at the origin is called a weak focus of order $k=$ $1,2, \ldots$ if $d(0)=d^{(1)}(0)=d^{(2)}(0)=\cdots=d^{(2 k)}(0)=0$, but $d^{(2 k+1)}(0) \neq 0$. Otherwise it is a weak focus of infinite order, i.e., a center. Bautin proved that a quadratic system with a weak focus of order $k<\infty$ may be perturbed in the coefficients $\lambda_{1}, \ldots, \lambda_{6}$ to produce $k$ limit cycles in a multiple bifurcation at the origin. Then starting from the Center Theorem, he recognized and dealt with a subtle point, viz., that by showing the Taylor coefficients of $d$ all lie in the ideal $\left(\lambda_{1}, v_{3}, v_{5}, v_{7}\right)$, in the ring $\mathbf{R}\left[\lambda_{1}, \ldots, \lambda_{6}\right]$ he could establish that no perturbation of a quadratic system produces more than three limit cycles near a weak focus of finite or infinite order.

Throughout this section we understand a plane vector field $\mathbf{X}$ to be given, and that $\mathbf{X}$ satisfies the following standing hypothesis:

The vector field $(x, y) \mapsto \mathbf{X}(x, y, \lambda)$ has a center at the origin for each $\lambda \in \mathbf{R}^{N},(x, y, \lambda) \mapsto \mathbf{X}(x, y, \lambda), x, y \in \mathbf{R}, \lambda \in \mathbf{R}^{N}$, is analytic, and the linearization of the vector field at this center is $(x, y) \mapsto(-y, x)$.

A center satisfying the linearization condition in the standing hypothesis is called a linear center. The standing hypothesis assures us that the period function associated with a vector field with a linear center will be analytic (cf. the Period Coefficient Lemma given below). We are interested in the generation of critical points of the period function at such a linear center. The linear center at the origin corresponding to the parameter value $\lambda_{*}$ is a weak center of order $k$ if

$$
\rho\left(\xi, \lambda_{*}\right):=P\left(\xi, \lambda_{*}\right)-P\left(0, \lambda_{*}\right)
$$


satisfies

$$
\rho\left(0, \lambda_{*}\right)=\rho^{\prime}\left(0, \lambda_{*}\right)=\cdots=\rho^{(2 k+1)}\left(0, \lambda_{*}\right)=0, \quad \rho^{(2 k+2)}\left(0, \lambda_{*}\right) \neq 0,
$$

where the derivatives indicated are taken with respect to the first argument of the function $\rho$. Otherwise, the linear center is a weak center of infinite order. Of course, a weak center of infinite order is an isochrone, i.e., all the periodic orbits surrounding the center have the same period. The analogue of the center theorem in the quadratic case is the content of Loud's theorem [27], which we will give a new proof of below. It gives the conditions for a quadratic system to be an isochrone. The remainder of this section is devoted to completing the analogy with Bautin's work by giving bounds for the number of critical periods which bifurcate from a weak center.

We call a period corresponding to a critical point of the period function which arises from a bifurcation from a weak center a local critical period. To be more precise, we say that $k$ local critical periods bifurcate from the weak center corresponding to the parameter value $\lambda_{*} \in \mathbb{R}^{N}$ if for every $\varepsilon>0$ and every neighborhood $W$ of $\lambda_{*}$ there is a point $\lambda^{1} \in W$ such that $P^{\prime}\left(\xi, \lambda^{1}\right)=0$ has $k$ solutions in $U:=(0, \varepsilon)$, where $P$ is the period function corresponding to the vector field $\mathbf{X}$. It should be noted here that we are counting only the positive solutions of $P^{\prime}(\xi, \lambda)=0$, because the nontrivial zeros of $P^{\prime}$ occur in pairs corresponding to the positive and negative intersections of the critical periodic orbits with the $x$-axis.

In order to study this bifurcation problem, we first record some of the properties of the period function $P$. The next lemma provides the basic structural information required for our analysis.

Lemma 2.1 [Period Coefficient Lemma]. If the vector field $\mathbf{X}$ satisfies the standing hypothesis, then the following statements about the corresponding period function $P$ are true.

(i) Define $P(0, \lambda)=2 \pi$ for $\lambda \in \mathbf{R}^{N}$. If $\lambda_{*} \in \mathbf{R}^{N}$, then there is an open neighborhood $W$ of $\lambda_{*}$ and an open interval $J$ containing $\xi=0$, such that the period function $(\xi, \lambda) \mapsto P(\xi, \lambda)$ is analytic on $\Omega:=J \times W$.

(ii) Given any $\lambda_{*} \in \mathbf{R}^{N}$, the period function $P$ is represented by its Taylor series

$$
P(\xi, \lambda)=2 \pi+\sum_{k=2}^{\infty} p_{k}(\lambda) \xi^{k},
$$

for $|\xi|$ and $\left|\lambda-\lambda_{*}\right|$ sufficiently small. Moreover, $p_{1} \equiv 0$ and for each $k \geq 2$, $p_{k} \in \mathbf{R}\left\{\lambda_{1}, \lambda_{2}, \ldots, \lambda_{N}\right\}_{\lambda_{*}}$, the ring of convergent power series at $\lambda_{*}$.

(iii) For $k \geq 1$ the Taylor coefficient $p_{2 k+1}$ belongs to the ideal $\left(p_{2}, p_{4}\right.$, $\left.\ldots, p_{2 k}\right)$ over the ring $\mathbf{R}\left\{\lambda_{1}, \lambda_{2}, \ldots, \lambda_{N}\right\}_{\lambda_{*}}$ for each $\lambda_{*} \in \mathbf{R}^{N}$. In particular, for any $\lambda \in \mathbf{R}^{N}$, the first $k \geq 1$ such that $p_{k}(\lambda) \neq 0$ is even. 
The proof of the lemma follows standard arguments and is omitted. The interested reader can find the necessary power series majorization methods in [29, p. $321 \mathrm{ff} ; 30$, pp. $104-107 ; 38$, pp. 84-87; and 40, $\S \S 4,21]$.

The next lemma is an immediate consequence of the Period Coefficient Lemma and the Weierstrass Preparation Theorem (cf. [9 or 14]); this proof is also omitted.

Lemma 2.2 [Finite Order Bifurcation Lemma]. If the vector field $\mathbf{X}$ satisfies the standing hypothesis, and if the weak center at the origin, corresponding to a parameter value $\lambda_{*}$, has order $k$, then no more than $k$ local critical periods bifurcate from this weak center at the parameter value $\lambda_{*}$.

We would like to determine conditions which would allow us to prove that the bound $k$ on the number of local critical periods given in the Finite Order Bifurcation Lemma is "tight", in the sense that these conditions would imply that if $n \leq k$, then there are perturbations of $\lambda_{*}$ for which precisely $n$ local critical periods bifurcate from the weak center corresponding to the parameter value $\lambda_{*}$. Such a result could be formulated and proved in the language of singularity theory, but since the space dimension is one, it is more appropriate to prove the result from elementary properties of functions of a single variable. However, we do need some definitions. Consider a finite set of functions $f_{i}: \mathbf{R}^{N} \rightarrow \mathbf{R}$, $i=1,2, \ldots, l$. The real (respectively, complex) variety $V\left(f_{1}, f_{2}, \ldots, f_{l}\right)$ is defined to be the set of $\lambda \in \mathbf{R}^{N}$ (respectively, $\mathbb{C}^{N}$ ) such that $f_{i}(\lambda)=0$ for $i=1,2, \ldots, l$. If $f: \mathbf{R}^{N} \rightarrow \mathbf{R}$, then we say that $f_{1}, f_{2}, \ldots, f_{l}$ are independent with respect to $f$ at $\lambda_{*} \in V\left(f_{1}, f_{2}, \ldots, f_{l}\right)$ if the following three conditions are satisfied:

(i) Every open neighborhood of $\lambda_{*}$ in $\mathbf{R}^{N}$ contains a point $\lambda$ such that $f_{l}(\lambda) \cdot f(\lambda)<0$.

(ii) The varieties $V\left(f_{1}, \ldots, f_{j}\right) 2 \leq j \leq l-1$, are such that if $\lambda \in$ $V\left(f_{1}, \ldots, f_{j}\right)$, and $f_{j+1}(\lambda) \neq 0$, then every neighborhood $W$ of $\lambda$ contains a point $\sigma \in V\left(f_{1}, \ldots, f_{j-1}\right)$ such that $f_{j}(\sigma) \cdot f_{j+1}(\lambda)<0$.

(iii) If $\lambda \in V\left(f_{1}\right)$ and $f_{2}(\lambda) \neq 0$, then every open neighborhood of $\lambda$ contains a point $\sigma$ such that $f_{1}(\sigma) \cdot f_{2}(\lambda)<0$.

Let $\lambda_{*} \in V\left(f_{1}, f_{2}, \ldots, f_{l}\right)$, and let $f$ be $C^{1}$ in a neighborhood of $\lambda_{*}$. Then it is easy to show, for example, that if the functions $f_{j}$ are $C^{1}$ on a neighborhood of $\lambda_{*}$, for $j=1,2, \ldots, l$, and if the vectors $\nabla f_{1}\left(\lambda_{*}\right), \ldots, \nabla f_{l}\left(\lambda_{*}\right)$, $\nabla f\left(\lambda_{*}\right)$ are linearly independent, then $f_{1}, f_{2}, \ldots, f_{l}$ are independent with respect to $f$ at $\lambda_{*}$. However, our definition of independence is substantially weaker than this, and it is this weaker condition which must be used in most of our applications.

Theorem 2.1 [Finite Order Bifurcation Theorem]. Let the vector field $\mathbf{X}$ satisfy the standing hypothesis, and have a weak center of order $k$ at the origin corresponding to the parameter value $\lambda_{*}$. If the Taylor coefficients $p_{2}, p_{4}, p_{6}, \ldots$, $p_{2 k}$ are independent with respect to $p_{2 k+2}$ at $\lambda_{*}$, then exactly $n$ critical periods 
bifurcate from the weak center corresponding to the parameter value $\lambda_{*}$ for any $n \leq k$.

Proof. Since $\mathbf{X}$ has a weak center of order $k$ at the origin when $\lambda=\lambda_{*}$ we have

$$
\rho\left(\xi, \lambda_{*}\right):=P(\xi, \lambda)-2 \pi=p_{2 k+2}\left(\lambda_{*}\right) \xi^{2 k+2}+\cdots,
$$

where $p_{2 k+2}\left(\lambda_{*}\right) \neq 0$. The Finite Order Bifurcation Lemma shows that at most $k$ critical periods can bifurcate from the weak center. We must show that we can obtain exactly $n$ critical periods for any $n \leq k$. However, it is clear from the definition of independence that it suffices to show the upper bound $k$ on the number of critical periods can be attained.

We assume $p_{2 k+2}\left(\lambda_{*}\right)>0$; the other case is similar. Choose a neighborhood $W$ about $\lambda_{*}$ and an $\varepsilon>0$, and define $U:=[0, \varepsilon)$. Since $p_{2 k+2}\left(\lambda_{*}\right)>0$, there is an $\varepsilon_{1}>0$ such that $U_{1}:=\left(0, \varepsilon_{1}\right) \subset U$, and $\rho\left(\xi, \lambda_{*}\right)>0$ for $\xi \in U_{1}$. Pick a $\xi_{1} \in U_{1}$, and then $\rho\left(\xi_{1}, \lambda_{*}\right)>0$. By the continuity of $\rho$ and the independence of the $p_{i}$ there is some $\lambda^{1} \in W$ such that $\rho\left(\xi_{1}, \lambda^{1}\right)>0$ and $p_{2}\left(\lambda^{1}\right)=p_{3}\left(\lambda^{1}\right)=\cdots=p_{2 k-2}\left(\lambda^{1}\right)=0$, but $p_{2 k}\left(\lambda^{1}\right)<0$. Then there is an $\varepsilon_{2}>0$ such that $U_{2}:=\left(0, \varepsilon_{2}\right) \subset U_{1}$, and $\rho\left(\xi, \lambda^{1}\right)<0$ for $\xi \in U_{2}$. It follows that there is at least one point $\eta_{1}$ such that $0<\eta_{1}<\xi_{1}$ and $\rho^{\prime}\left(\eta_{1}, \lambda^{1}\right)=0$. We can repeat this process by choosing a point $\xi_{2}$ such that $0<\xi_{2}<\eta_{1}<\xi_{1}$ where $\rho\left(\xi_{2}, \lambda^{1}\right)<0$. By the independence condition, we can pick a point $\lambda^{2}$ in $W$ sufficiently close to $\lambda^{1}$ so that $\rho\left(\xi_{2}, \lambda^{2}\right)<0, \rho\left(\xi_{1}, \lambda^{2}\right)>0$, and $p_{2}\left(\lambda^{2}\right)=\cdots=p_{2 k-4}\left(\lambda^{2}\right)=0$ but $p_{2 k-2}\left(\lambda^{2}\right)>0$. Hence, $\rho\left(\xi, \lambda^{2}\right)=0$ has at least three different solutions in $[0, \varepsilon)$, and thus there will be at least two points in $U$ where $\rho^{\prime}$ vanishes. This process can be repeated $k$ times after which there are at least $k$ critical periods in $U$ corresponding to a suitable $\lambda^{k} \in W$. Combining this result with the Finite Order Bifurcation Lemma we have the desired conclusion.

Up to this point we have only considered bifurcation from a weak center of finite order. The case of a weak center of infinite order, i.e., an isochrone, is much more delicate. Here we start with a parameter value $\lambda_{*}$ such that $(x, y) \mapsto \mathbf{X}\left(x, y, \lambda_{*}\right)$ is an isochrone. It follows from the Period Coefficient Lemma that the Taylor coefficients $p_{k}$ of the period function are in the ring $R\left\{\lambda_{1}, \lambda_{2}, \ldots, \lambda_{N}\right\}_{\lambda_{*}}$ of convergent power series at $\lambda_{*}$. Since this ring is Noetherian [24] the ideal generated by all the Taylor coefficients of the period function of $\mathbf{X}$ has a finite basis. In fact, taking into account the Period Coefficient Lemma (iii), it follows that there is an integer $k$ such that all Taylor coefficients are in the ideal $\left(p_{2}, p_{4}, \ldots, p_{2 k+2}\right)$. Once this value of $k$ is determined, the following theorem can be used to analyze our bifurcation problem in a neighborhood of the isochrone corresponding to $\lambda_{*}$.

Theorem 2.2 [Isochrone Bifurcation Theorem]. Let the vector field $\mathbf{X}$ satisfy the standing hypothesis. If $\mathbf{X}$ has an isochronous center at the origin corresponding 
to the parameter value $\lambda_{*}$ and if all Taylor coefficients of the function $\rho:=$ $P-2 \pi$ are in the ideal $\left(p_{2}, p_{4}, \ldots, p_{2 k+2}\right)$ over $\mathbf{R}\left\{\lambda_{1}, \lambda_{2}, \ldots, \lambda_{N}\right\}_{\lambda_{*}}$, the ring of convergent power series at $\lambda_{*}$, then there are at most $k$ local critical periods which bifurcate from the isochronous center at $\lambda_{*}$. Moreover, if $p_{2}, p_{4}, \ldots, p_{2 k}$ are independent with respect to $p_{2 k+2}$ at $\lambda_{*}$, then exactly $n$ local critical periods bifurcate from the center at the parameter value $\lambda_{*}$ for each $n \leq k$.

Proof. We can without loss of generality assume that $\lambda_{*}$ is the origin of our coordinate system. Thus our ring is $\mathbf{R}\left\{\lambda_{1}, \lambda_{2}, \ldots, \lambda_{N}\right\}:=\mathbf{R}\left\{\lambda_{1}, \lambda_{2}, \ldots, \lambda_{N}\right\}_{0}$. Consider the power series representation of $\rho$ :

$$
\rho(\xi, \lambda)=\sum_{\nu=0}^{\infty} p_{\nu+2}(\lambda) \xi^{\nu+2} .
$$

Let $\Lambda(\varepsilon)$ denote the closed ball in $\mathbf{R}^{N}$ of radius $\varepsilon>0$ with center at $\mathbf{0} \in \mathbf{R}^{N}$. If $f \in \mathbf{R}\left\{\lambda_{1}, \lambda_{2}, \ldots, \lambda_{N}\right\}$, and $\varepsilon>0$ is sufficiently small, then we define

$$
\|f\|_{\Lambda(\varepsilon)}:=\sup _{\lambda \in \Lambda(\varepsilon)}|f(\lambda)| .
$$

Let $S$ denote the index set consisting of all integers $\nu>2 k$ and all odd integers $2 i-1$ for $i=1,2, \ldots, k$. By hypothesis, $p_{\nu+2} \in\left(p_{2}, p_{4}, \ldots, p_{2 k+2}\right)$ for $\nu \in \mathrm{S}$, and therefore $p_{\nu+2}$ can be written in the form

$$
p_{\nu+2}=\sum_{j=0}^{k} \alpha_{\nu+2, j} p_{2 j+2},
$$

where each $\alpha_{\nu+2, j} \in \mathbf{R}\left\{\lambda_{1}, \lambda_{2}, \ldots, \lambda_{N}\right\}$. An easy corollary to a result in Hervé [24, Theorem 7, p. 32] enables us to conclude that a representation of the above form exists where the $\alpha_{\nu+2, j}$ have the additional property, that for $\varepsilon>0$ sufficiently small, there is an $M>0$ such that

$$
\left\|\alpha_{\nu+2, j}\right\|_{\Lambda(\varepsilon)} \leq M\left\|p_{\nu+2}\right\|_{\Lambda(\varepsilon)} .
$$

Combining these estimates with the Cauchy inequalities, one can show that the series

$$
\sum_{\nu \in \mathbf{S}} \alpha_{\nu+2, j}(\lambda) \xi^{\nu+2}
$$

is convergent for $j=0,1, \ldots, k$. Thus an interchange of the order of summation can be justified to write

$$
\rho(\xi, \lambda)=\sum_{j=0}^{k} p_{2 j+2}(\lambda) \xi^{2 j+2}+\sum_{j=0}^{k} p_{2 j+2}(\lambda) \sum_{\nu \in \mathbf{S}} \alpha_{\nu+2, j}(\lambda) \xi^{\nu+2} .
$$

Now by part (iii) of the Period Coefficient Lemma, we may assume that $\alpha_{2 i+1, j}$ $\equiv 0$ for the finite set of indices $i \leq j \leq k$, for $i=1,2, \ldots, k$. It follows that there exist $\varepsilon, \delta>0$ such that for $|\xi|<\delta$ and $\lambda \in \Lambda(\varepsilon)$, the series for $\rho(\xi, \lambda)$ can be rewritten as

$$
\rho(\xi, \lambda)=\sum_{j=0}^{k} p_{2 j+2}(\lambda) \xi^{2 j+2}\left(1+\Psi_{2 j+2}(\xi, \lambda)\right),
$$


where $\Psi_{2 j+2}, j=0,1,2, \ldots$, are analytic functions of $\xi$ and $\lambda$, which vanish at $\xi=0$. We suppress mention of $\lambda$, differentiate with respect to $\xi$, and find that the function $\rho^{\prime}$ has the form

$$
\rho^{\prime}(\xi)=\sum_{j=0}^{k}(2 j+2) p_{2 j+2} \xi^{2 j+1}\left(1+\psi_{2 j+2}(\xi)\right),
$$

where again the $\psi_{2 j+2}(0)=0$.

The proof proceeds just like the proof constructed by Bautin [5] of the analogous fact about limit cycles. For the proof we may choose $\varepsilon$ and $\delta$ smaller, if necessary, so that in the new neighborhoods we have convergence and also

$$
1+\psi_{2}(\xi)>\frac{1}{2} \text {. }
$$

Then $\rho^{\prime}$ has the same number of positive zeros as the function obtained by dividing its series representation by $\xi\left(1+\psi_{2}(\xi)\right)$ which defines a new analytic function of the form

$$
\tau(\xi)=2 p_{2}+4 p_{4} \xi^{2}\left(1+\Phi_{4}(\xi)\right)+\cdots+(2 k+2) p_{2 k+2} \xi^{2 k}\left(1+\Phi_{2 k+2}(\xi)\right) .
$$

But then $\rho^{\prime}$ has at most one more isolated positive zero than

$$
\tau^{\prime}(\xi)=8 p_{4} \xi\left(1+\phi_{4}(\xi)\right)+\cdots+(2 k+2)(2 k) p_{2 k+2} \xi^{2 k-1}\left(1+\phi_{2 k+2}(\xi)\right) .
$$

Iterating, we may choose $\varepsilon$ and $\delta$, smaller if necessary, so

$$
1+\phi_{4}(\xi)>\frac{1}{2}
$$

and, as before, $\tau^{\prime}$ has the same number of positive zeros as the function $\eta$ which results after division of $\tau^{\prime}$ by $\xi\left(1+\phi_{4}(\xi)\right)$. So, we may repeat the process.

Note that after $k$ interations we obtain the derivative of a certain function $\omega$ and this derivative has the form

$$
\omega^{\prime}(\xi)=(2 k+2)(2 k) \cdots 2 p_{2 k+2} \xi^{i}(1+\chi(\xi)),
$$

where $i=1$ if $k$ is odd and $i=2$ if $k$ is even. Moreover, the number of positive zeros of $\rho^{\prime}$ exceeds the number of positive zeros of $\omega^{\prime}$ by at most $k$. But, there is a final choice of $\varepsilon$ and $\delta$ so that

$$
1+\chi(\xi)>\frac{1}{2}
$$

and we see that $\omega^{\prime}$ has no positive isolated zeros. Thus, for any $\lambda_{*}$ there is a neighborhood of the origin in $\xi$ space and a neighborhood of $\lambda_{*}$ in the parameter space where $\rho^{\prime}$ has at most $k$ isolated positive zeros.

For the last statement of the theorem we note that the polynomials $p_{2}, p_{4}$, $p_{6}, \ldots, p_{2 k}$ are independent with respect to $p_{2 k+2}$ at $\lambda_{*}$, and thus for any neighborhood $W$ of $\lambda_{*}$ there is a $\lambda_{W} \in W$ such that the vector field $(x, y) \mapsto$ $\mathbf{X}(x, y, \lambda)$ has a weak center of order $k$ at the origin. Then the conclusion follows directly from the Finite Order Bifurcation Theorem. 
Remark. The precise statement of the Isochrone Bifurcation Theorem is used in this paper to analyze our bifurcation problem which contains some special features. However, with the same method of proof one can easily show a similar result for any analytic bifurcation equation $F(\xi, \lambda)=0$, with $\xi \in \mathbf{R}$ and $\lambda:=\left(\lambda_{1}, \lambda_{2}, \ldots \lambda_{N}\right) \in \mathbf{R}^{N}$. To wit, assume $F$ has the form

$$
F(\xi, \lambda)=a_{0}(\lambda)+a_{1}(\lambda) \xi+a_{2}(\lambda) \xi^{2}+\cdots .
$$

If $F\left(\xi, \lambda_{*}\right) \equiv 0$ and if the ideal in $\mathbf{R}\left\{\lambda_{1}, \lambda_{2}, \ldots, \lambda_{N}\right\}_{\lambda_{*}}$ generated by all the $a_{k}$ for $k \geq 0$ is equal to the ideal $\left(a_{0}, a_{1}, \ldots, a_{K}\right)$ then there exist $\varepsilon, \delta>0$ such that for each $\lambda$ satisfying $0<$ $\left|\lambda-\lambda_{*}\right|<\delta$, the equation $F(\xi, \lambda)=0$ has at most $K$ solutions, $\xi \in(-\varepsilon, \varepsilon)$.

The following example illustrates why we must analyze the ideal of all Taylor coefficients in order to obtain the maximum number of critical periods which bifurcate from an isochrone.

EXAMPLE. Let the function $\left(x, \lambda_{1}, \lambda_{2}\right) \mapsto F\left(x, \lambda_{1}, \lambda_{2}\right)$ be defined by

$$
F\left(x, \lambda_{1}, \lambda_{2}\right)=\left(\lambda_{2}-\lambda_{1}^{2}\right) x+\lambda_{2} x^{2}-\lambda_{1} x^{3} .
$$

We consider bifurcation of the zeros of the function $F$. Note that the ideal $\mathfrak{m}=\left(\lambda_{2}-\lambda_{1}^{2}, \lambda_{2}\right)$ contains all the "isochrones", that is, $V(\mathfrak{m})=\{0\}$, and $F(x, 0,0) \equiv 0$. One might conclude that the 2-jet of $F$, namely

$$
j^{2} F\left(x, \lambda_{1}, \lambda_{2}\right)=\left(\lambda_{2}-\lambda_{1}^{2}\right) x+\lambda_{2} x^{2},
$$

contains all the information for the bifurcation problem, and thus conclude that at most one zero bifurcates from the origin (only nonzero roots are counted). But this is not true. To see this, consider the function $F$ along the parabola $\lambda_{1}=t, \lambda_{2}=2 t^{2}$. Then

$$
F\left(x, t, 2 t^{2}\right)=t x\left(t+2 t x-x^{2}\right),
$$

and two real nonzero roots bifurcate from the origin at $t=0$. The explanation is simply that the third Taylor coefficient $-\lambda_{1}$ is not in the ideal $\mathfrak{m}$.

The use of the Isochrone Bifurcation Theorem requires some description of the ideal $\mathfrak{m}:=\left(p_{2}, p_{3}, \ldots\right)$ of all the period coefficients, i.e., the Taylor coefficients $($ at $\xi=0)$ of the function $\rho(\xi, \lambda)=P(\xi, \lambda)-2 \pi$. We note that by the Period Coefficient Lemma, the ideal $\mathfrak{m}$ is generated by $\left(p_{2}, p_{4}, \ldots\right)$. Since the rings we are working with are all Noetherian, there is a least positive integer $k=k(\mathfrak{m})<\infty$ such that $\mathfrak{m}_{k}:=\left(p_{2}, p_{4}, \ldots, p_{2 k+2}\right)=\mathfrak{m}$. The task then, is to find $k$. In the applications of the bifurcation theorems which appear in the subsequent sections of this paper, the coefficients $p_{k}$ described in the Period Coefficient Lemma always turn out to be elements of the real polynomial ring $R\left[\lambda_{1}, \lambda_{2}, \ldots, \lambda_{N}\right]$ (we will see, momentarily, that in this case, working in a local power series ring still has a point). For any $n \geq 0$ define 
$\mathfrak{m}_{n}=\left(p_{2}, p_{4}, \ldots, p_{2 n+2}\right)$ in $\mathbf{R}\left[\lambda_{1}, \lambda_{2}, \ldots, \lambda_{N}\right]$, and let $V\left(\mathfrak{m}_{n}\right)$ be the corresponding complex variety. When working with polynomial period coefficients, the following simple approach to calculating $k=k(\mathfrak{m})$ often succeeds.

(i) Find the first integer $l$ such that $V\left(\mathfrak{m}_{l+1}\right)=V\left(\mathfrak{m}_{l}\right)$.

(ii) Calculate, using, for example, a computer algebra implementation of Buchberger's algorithm [10], the first $k \geq l$ for which $p_{2(k+1)+2} \in \mathfrak{m}_{k}$.

(iii) Determine an algebraic ideal membership criterion for $\mathfrak{m}_{k}$.

(iv) Prove $p_{n}$ satisfies the criterion of (iii) for $n>2 k+2$.

The ideal membership problem of step (iii), according to van der Waerden [50, p. 159], is the "main problem in the ideal theory of polynomial domains". Although there are algorithms for deciding polynomial ideal membership, e.g. [10], we must find ideal membership criteria in a simple analytic form if we hope to apply them to the period coefficients $p_{n}$. Indeed, our solutions to step (iii) involve either finding differential ideal membership conditions which can be verified by an analysis of the differential equations (cf. $\S 3$ ), or finding algebraic criteria for testing membership which can be verified by analyzing a recursive scheme for generating the period coefficients (cf. §4).

Once the outlined procedure is carried out we have an upper bound for the number of critical periods which may bifurcate from the isochrone. However, if the finite set of generators for the ideal are not independent, the upper bound may not be sharp. Nonetheless, in some cases we can get a tighter bound by working in a ring larger than $\mathbf{R}\left[\lambda_{1}, \lambda_{2}, \ldots, \lambda_{N}\right]$. For example, in the First Local Ideal Membership Theorem of $\xi 3$, we find it useful to analyze the ideal $\mathfrak{m}_{k}$ in the local ring

$$
\mathbf{R}\left[\lambda_{1}, \lambda_{2}, \ldots, \lambda_{N}\right]_{\lambda_{*}}:=\left\{f / g \mid f, g \in \mathbf{R}\left[\lambda_{1}, \lambda_{2}, \ldots, \lambda_{N}\right] \text { and } g\left(\lambda_{*}\right) \neq 0\right\},
$$

and use the full force of the Isochrone Bifurcation Theorem.

Unfortunately, it is not always possible to improve the upper bound by passing to a larger ring. For example, in $\S 4$, one finds that the complex variety $V(\mathfrak{m})=\{\mathbf{0}\}$, and that, generally, $V\left(\mathfrak{m}_{l}\right)=\{\mathbf{0}\}$ for some $l<k=k(\mathfrak{m})$. In such a case, the independence condition in the Isochrone Bifurcation Theorem cannot be satisfied, and the following proposition shows that passing to a larger ring will not help.

Proposition 2.1. Let $f, f_{1}, f_{2}, \ldots, f_{k}$ be polynomials and let $I$ denote the ideal generated by $f_{1}, f_{2}, \ldots, f_{k}$ in $\mathbb{C}\left[\lambda_{1}, \lambda_{2}, \ldots, \lambda_{N}\right]$. If $V(I)=\{\mathbf{0}\}$, and if $f=\alpha_{1} f_{1}+\alpha_{2} f_{2}+\cdots+\alpha_{k} f_{k}$, where the $\alpha_{i}, i=1,2, \ldots, k$, are formal power series in $\mathbb{C}\left[\left[\lambda_{1}, \lambda_{2}, \ldots, \lambda_{N}\right]\right]$, then $f \in I$.

Proof. For each $j$, the monomial $\lambda_{j}$ vanishes on $V(I)$. By Hilbert's Nullstellensatz, some power $r_{j}$ of $\lambda_{j}$ is in $I$. If $d:=N \max \left\{r_{j} \mid j=1,2, \ldots, N\right\}$ and $u:=\lambda_{1}^{\sigma_{1}} \lambda_{2}^{\sigma_{2}} \cdots \lambda_{N}^{\sigma_{N}}$, where $\operatorname{deg} u=\sigma_{1}+\sigma_{2}+\cdots+\sigma_{N}>d$, then some $\sigma_{j}$ exceeds $r_{j}$ and the power product $u \in I$. Now we can write $f$ in the form

$$
f=A_{1} f_{1}+A_{2} f_{2}+\cdots+A_{k} f_{k}+R,
$$


where each $A_{i}$ is a polynomial made up of the terms in the power series $\alpha_{i}$ with degree not exceeding $d$. Since $f$ and $A_{1} f_{1}+A_{2} f_{2}+\cdots+A_{k} f_{k}$ are polynomials, so is $R$. But all terms of $R$ have degree larger than $d$. Thus, $R \in I$. Since $A_{1} f_{1}+A_{2} f_{2}+\cdots+A_{k} f_{k}$ is in $I$ it follows that $f \in I$.

In some cases, the bifurcation problem at an isochrone can be treated by elementary considerations which do not involve analyzing the ideal of all Taylor coefficients of $\rho$. The following lemma is one such case, and it will be needed in the next section.

Lemma 2.3 [Positive Lemma]. Let $(\xi, \lambda) \mapsto F(\xi, \lambda)$ be an analytic function of the form

$$
F(\xi, \lambda)=a_{2}(\lambda)+a_{3}(\lambda) \xi+a_{4}(\lambda) \xi^{2}+\cdots,
$$

where $\xi \in \mathbf{R}$ and $\lambda \in \mathbf{R}^{N}$. If each $a_{k}, k \geq 2$, is a homogeneous polynomial, $a_{2}$ is positive definite, and if $\operatorname{deg} a_{k}>\operatorname{deg} a_{2}$ for $k>2$, then there exist $\varepsilon, \delta>0$ such that for each $\lambda \in \mathbf{R}^{N}$ satisfying $0<|\lambda|<\delta$ the equation $F(\xi, \lambda)=0$ has no solution, $\xi \in(0, \varepsilon)$.

Proof. An elementary argument will show that the function defined by

$$
H(\xi, \lambda):=\left[\begin{array}{ll}
1 & \text { if } \lambda=\mathbf{0} \\
F(\xi, \lambda) / a_{2}(\lambda) & \text { if } \lambda \neq \mathbf{0}
\end{array}\right.
$$

is continuous on some compact neighborhood of the origin in $\mathbf{R} \times \mathbf{R}^{N}$. The desired conclusion follows.

\section{QUADRATIC SYSTEMS}

In this section we specialize to the bifurcation of critical periods from weak centers of quadratic systems. To formulate our results we recall first that any quadratic system with a center at the origin can be transformed by a linear change of coordinates to a system $\mathbf{B}_{\lambda}$ in Bautin's form:

$$
\begin{aligned}
& \dot{x}=-y-\lambda_{3} x^{2}+\left(2 \lambda_{2}+\lambda_{5}\right) x y+\lambda_{6} y^{2}, \\
& \dot{y}=x+\lambda_{2} x^{2}+\left(2 \lambda_{3}+\lambda_{4}\right) x y-\lambda_{2} y^{2},
\end{aligned}
$$

where $\lambda$ is in the Bautin variety $\mathbf{B V}$, which we define to be the union of the sets

$$
\begin{aligned}
& \mathfrak{B}_{1}=\left\{\lambda \mid \lambda_{4}=\lambda_{5}=0\right\}, \\
& \mathfrak{B}_{2}=\left\{\lambda \mid \lambda_{3}=\lambda_{6}\right\}, \\
& \mathfrak{B}_{3}=\left\{\lambda \mid \lambda_{5}=\lambda_{4}+5 \lambda_{3}-5 \lambda_{6}=\lambda_{3} \lambda_{6}-2 \lambda_{6}^{2}-\lambda_{2}^{2}=0\right\}, \\
& \mathfrak{B}_{4}=\left\{\lambda \mid \lambda_{2}=\lambda_{5}=0\right\} .
\end{aligned}
$$

We consider a point $\lambda_{*} \in \mathbf{B V}$ which corresponds to a quadratic system with a weak center and study the bifurcations of critical periods for perturbations of $\lambda_{*}$ which remain in $\mathbf{B V}$. We will obtain a quite detailed knowledge of the bifurcations which occur in the various families of quadratic systems whose parameters lie in $\mathbf{B V}$, but our main result is given in the next theorem. 
Theorem 3.1 [Quadratic Weak Center Bifurcation Theorem].

(i) If a quadratic system has a weak center of finite order, then at most two local critical periods bifurcate from this center. Moreover, there are perturbations of the weak centers of order one with one critical period and there are perturbations of the weak centers of order two with exactly one or exactly two critical points.

(ii) At most one critical period can bifurcate from an isochronous center of a nonlinear quadratic system. At most two critical periods can bifurcate from the linear isochrone. In both cases there are perturbations in $\mathbf{B V}$ with the maximum number of critical periods.

The remainder of this section will be devoted to developing the results needed for the proof of this theorem. We will identify the weak centers of finite order and the isochrones which correspond to points in BV. The perturbations from these points lie in one of the four subvarieties $\mathfrak{B}_{k}$. Thus, we will analyze these perturbations in families of vector fields $(x, y) \mapsto \mathbf{X}(x, y, \lambda)$ corresponding to a Bautin system $\mathbf{B}_{\lambda}$, where $\lambda \in \mathbf{B V}$. In order to do this using the results of $\S 2$, we must use suitable parametrizations of the vector fields $(x, y) \mapsto \mathbf{X}(x, y, \lambda)$ which will have centers for all values of the parameters in a suitable vector space $\mathbf{R}^{N}$. The varieties $\mathfrak{B}_{1}, \mathfrak{B}_{2}$, and $\mathfrak{B}_{4}$ are vector subspaces of the full parameter space, so that vector fields with parameters in these varieties are parametrized by the obvious restrictions. However, the case $\lambda \in \mathfrak{B}_{4}$ will turn out to be especially important in the proof of the Quadratic Weak Center Bifurcation Theorem. In this case, we note that after a rotation of coordinates, the corresponding Bautin system $\mathbf{B}_{\lambda}$ can be put in the form of Loud's system $\mathbf{L}$ [27]:

$$
\dot{x}=-y+B x y, \quad \dot{y}=x+D x^{2}+F y^{2},
$$

and it will be convenient to use this parametrization of the Bautin vector fields on $\mathfrak{B}_{4}$. We will also refer to the special case of Loud's system, where $B=1$, as the dehomogenized Loud system $\mathbf{L}$. For the nonlinear homogeneous subvariety $\mathfrak{B}_{3}$ we obtain two analytic families of vector fields which correspond to the two nappes of the cone defined by $\mathfrak{B}_{3}$. In particular, we decompose $\mathfrak{B}_{3}$ into the union of two sets

$$
\begin{aligned}
& \mathfrak{B}_{3}^{+}:=\left\{\lambda \mid \lambda_{2}=\sigma \tau, \lambda_{3}=\sigma^{2}+2 \tau^{2}, \lambda_{4}=-5\left(\sigma^{2}+\tau^{2}\right), \lambda_{5}=0, \lambda_{6}=\tau^{2}\right\}, \\
& \mathfrak{B}_{3}^{-}:=\left\{\lambda \mid \lambda_{2}=\sigma \tau, \lambda_{3}=-\left(\sigma^{2}+2 \tau^{2}\right), \lambda_{4}=5\left(\sigma^{2}+\tau^{2}\right), \lambda_{5}=0, \lambda_{6}=-\tau^{2}\right\},
\end{aligned}
$$

so the corresponding vector fields are parametrized by $\mathbf{R}^{2}$. Note that the polynomials in $(\sigma, \tau)$ which give the parametrizations of $\mathfrak{B}_{3}^{ \pm}$are all homogeneous of degree two.

Our first task is to identify the isochrones and the weak centers of finite order for the Bautin systems $\mathbf{B}_{\lambda}$. For this and the rest of our analysis a knowledge of the coefficients of the period functions of the various families of vector fields is crucial. Throughout this section the period function $\xi \mapsto P(\xi, \lambda)$ is represented 
as a power series

$$
P(\xi, \lambda)=2 \pi+p_{2}(\lambda) \xi^{2}+p_{3}(\lambda) \xi^{3}+\cdots,
$$

in accordance with the Period Coefficient Lemma in $\S 2$. The next lemma gives some of the basic facts about the period coefficients for the families of quadratic vector fields which we will use for the bifurcation analysis in this section.

Lemma 3.1 [Quadratic Period Coefficient Lemma].

(i) If the plane quadratic vector field $(x, y) \mapsto \mathbf{X}(x, y, \lambda)$ is in the Bautin form $\mathbf{B}_{\lambda}$ for $\lambda \in \mathfrak{B}_{k}$, then the corresponding period coefficients $p_{k}$ are homogeneous polynomials in the parameters defining the variety $\mathfrak{B}_{k}$. If $j>k$, then the degree of $p_{j}$ is greater than the degree of $p_{k}$. Moreover, the coefficient $p_{2}$ can be obtained on any one of the varieties $\mathfrak{B}_{k}$ by substituting the parametrization of $\mathfrak{B}_{k}$ into the expression

$$
p_{2}(\lambda)=\frac{\pi}{12}\left(16 \lambda_{2}^{2}+8 \lambda_{2} \lambda_{5}+\lambda_{5}^{2}+18 \lambda_{3}^{2}-12 \lambda_{3} \lambda_{6}+9 \lambda_{3} \lambda_{4}+10 \lambda_{6}^{2}-\lambda_{4} \lambda_{6}+\lambda_{4}^{2}\right) .
$$

(ii) For Loud's system $\mathbf{L}$ the period coefficients $p_{2}, p_{4}, p_{6}$, dehomogenized in the first variable, i.e., $B=1$, are given by

$$
\begin{aligned}
& p_{2}(1, D, F)=\frac{\pi}{12}\left(10 D^{2}\right.\left.+10 D F-D+4 F^{2}-5 F+1\right) \\
& p_{4}(1, D, F)=\frac{\pi}{1152}\left(1540 D^{4}+4040 D^{3} F+1180 D^{3}+4692 D^{2} F^{2}\right. \\
&+ 1992 D^{2} F+453 D^{2}+2768 D F^{3}+228 D F^{2} \\
&+\left.318 D F-2 D+784 F^{4}-616 F^{3}-63 F^{2}-154 F+49\right), \\
& p_{6}(1, D, F)=\frac{\pi}{1244160}( 142600 D^{6}+17971800 D^{5} F \\
&+6780900 D^{5}+34474440 D^{4} F^{2}+22992060 D^{4} F \\
&+4531170 D^{4}+37257320 D^{3} F^{3}+28795260 D^{3} F^{2} \\
&+10577130 D^{3} F+1491415 D^{3}+24997584 D^{2} F^{4} \\
&+14770932 D^{2} F^{3}+7686378 D^{2} F^{2}+2238981 D^{2} F \\
&+339501 D^{2}+10527072 D F^{5}+367584 D F^{4} \\
&+1400478 D F^{3}+598629 D F^{2}+228900 D F \\
&-663 D+2302784 F^{6}-1830576 F^{5}-213972 F^{4} \\
&\left.-126313 F^{3}-53493 F^{2}-114411 F+35981\right) .
\end{aligned}
$$

Proof. For reasons of space we have only displayed the dehomogenized polynomials for Loud's system $\mathbf{L}$. The full set of polynomials can be obtained by homogenization, i.e., $p_{k}(B, D, F)=B^{k} p_{k}(1, D / B, F / B)$.

By using polar coordinates (and the appropriate parametrization for $\lambda$ ) the systems of differential equations in the lemma can all be represented in the following form:

$$
\dot{r}=r^{2} f(\theta, \lambda), \quad \dot{\theta}=1+r g(\theta, \lambda),
$$


for suitable functions $f$ and $g$. Then the corresponding period function has the following integral representation:

$$
P(\xi, \lambda)=\int_{0}^{2 \pi} \frac{1}{1+r(\theta, \xi, \lambda) g(\theta, \lambda)} d \theta,
$$

where $\theta \mapsto r(\theta, \xi, \lambda)$ is the solution of the initial value problem

$$
\frac{d r}{d \theta}=\frac{r^{2} f(\theta, \lambda)}{1+r g(\theta, \lambda)}, \quad r(0, \xi)=\xi .
$$

The Taylor coefficients of $P$ can then be calculated using the algorithm in Appendix B and a convenient computer algebra package such as REDUCE or MACSYMA. The polynomials displayed in the lemma are obtained by just such a calculation.

To show the period coefficients are homogeneous polynomials, we choose one of the subvarieties $\mathfrak{B}_{k}$. There is a parametrization of $\lambda$ in this variety so that the period function is given by the above formula for all values of the parameters in a certain vector space. As in the proof of the Period Coefficient Lemma ( $\S 2)$, the function $\xi \mapsto r(\theta, \xi, \lambda)$ can be expanded in a power series

$$
r(\theta, \xi, \lambda)=\sum_{k=1}^{\infty} u_{k}(\theta, \lambda) \xi^{k},
$$

which converges for $0 \leq \theta \leq 2 \pi$ and $\xi$ sufficiently small. Using the initial condition, it is not difficult to verify that $u_{1}(\theta) \equiv 1$. Moreover, we know by the argument of Bautin [5, pp. 5-6] that the $u_{j}(\theta, \lambda)$ are polynomials in $\sin \theta$ and $\cos \theta$ with coefficients which are homogeneous of degree $j-1$ in $\lambda$, i.e., in the variables $\lambda_{2}, \lambda_{3}, \ldots, \lambda_{6}$.

Now we compute

$$
\frac{d t}{d \theta}=1+\sum_{k=1}^{\infty}(-1)^{k} g^{k}(\theta, \lambda) r(\theta, \xi, \lambda)^{k} .
$$

Then, following P. Henrici [25, p. 36], we substitute the series for $r(\theta, \xi, \lambda)$ into the preceding series, and find that

$$
\frac{d t}{d \theta}=1+\sum_{j=1}^{\infty} c_{j}(\theta, \lambda) \xi^{j}
$$

where

$$
c_{j}(\theta, \lambda)=\sum_{k=1}^{j}(-1)^{k} g^{k}(\theta, \lambda) b_{j}^{(k)}(\theta, \lambda),
$$

with the coefficients $b_{j}^{(k)}$ determined by the relation

$$
\left(\xi+\sum_{l=2}^{\infty} u_{l}(\theta, \lambda) \xi^{l}\right)^{k}=\sum_{j=1}^{\infty} b_{j}^{(k)}(\theta, \lambda) \xi^{j}
$$


In order to calculate the $b_{j}^{(k)}$, we first generate a sequence $d_{n}^{(k)}$ determined by

$$
\sum_{n=0}^{\infty} d_{n}^{(k)}(\theta, \lambda) \xi^{n+k}=\left(\xi+\sum_{l=2}^{\infty} u_{l}(\theta, \lambda) \xi^{l}\right)^{k}=\xi^{k}\left(1+\sum_{l=1}^{\infty} u_{l+1}(\theta, \lambda) \xi^{l}\right)^{k}
$$

Then by the J. C. P. Miller formula [25, p. 42], we have

$$
d_{n}^{(k)}(\theta, \lambda)=\frac{1}{n} \sum_{i=1}^{n}[(k+1) i-n] d_{n-i}^{(k)}(\theta, \lambda) u_{i+1}(\theta, \lambda),
$$

where $d_{0}^{(k)}(\theta, \lambda) \equiv 1$. An easy induction argument shows that $d_{n}^{(k)}(\theta, \lambda)$ is homogeneous of degree $n$ in $\lambda$. Since $b_{j}^{(k)}=d_{j-k}^{(k)}$, it follows that $b_{j}^{(k)}$ is homogeneous of degree $j-k$ in $\lambda$ for $k \leq j$.

In view of the fact that $g(\theta, \lambda)$ is linear in $\lambda$, and the fact that the $b_{j}^{(k)}$ for $k \leq j$ are homogeneous of degree $j-k$ in $\lambda$, it follows that $c_{j}$ is homogeneous of degree $j$ in $\lambda$. Since

$$
p_{j}(\lambda)=\int_{0}^{2 \pi} c_{j}(\theta, \lambda) d \theta
$$

it follows that $p_{j}$ is homogeneous of degree $k$ in $\left(\lambda_{2}, \lambda_{3}, \ldots, \lambda_{6}\right)$. This proves the result when $\lambda$ is constrained to stay in one of the subspaces $\mathfrak{B}_{1}, \mathfrak{B}_{2}$, or $\mathfrak{B}_{4}$. If $\lambda$ is constrained to stay in the homogeneous variety $\mathfrak{B}_{3}$, or more precisely if $\lambda_{5}=0$, and $\lambda_{2}, \lambda_{3}, \lambda_{4}, \lambda_{6}$, are parametrized by the homogeneous polynomials (of degree 2) specified in the definitions of $\mathfrak{B}_{3}^{ \pm}$, then $p_{j}$ is a homogeneous polynomial in $(\sigma, \tau)$ of degree $2 j$.

For quadratic systems the isochrones were completely determined by W. Loud [27]. His theorem actually contains more information, since it also identifies the weak centers of finite order. We give here a modification of Loud's Theorem which makes this information explicit. Although our proof is similar to the original, we replace an appeal to Urabe's Theorem $[45,46]$ by a direct computation of the period coefficients.

Theorem 3.2 [Loud's Theorem] [27]. The quadratic system in Bautin's form

$$
\begin{aligned}
& \dot{x}=-y-\lambda_{3} x^{2}+\left(2 \lambda_{2}+\lambda_{5}\right) x y+\lambda_{6} y^{2}, \\
& \dot{y}=x+\lambda_{2} x^{2}+\left(2 \lambda_{3}+\lambda_{4}\right) x y-\lambda_{2} y^{2}
\end{aligned}
$$

has an isochronous center at $(0,0)$ if and only if either the system is linear or the axes can be rotated to bring the system to the form

$$
\dot{x}=-y+x y, \quad \dot{y}=x+D x^{2}+F y^{2},
$$

and the point $(D, F) \in \mathbf{R}^{2}$ coincides with one of the following four points:

$$
I_{1}(0,1), \quad I_{2}\left(-\frac{1}{2}, 2\right), \quad I_{3}\left(0, \frac{1}{4}\right), \quad \text { or } \quad I_{4}\left(-\frac{1}{2}, \frac{1}{2}\right) \text {. }
$$

In addition, if $\lambda \in \mathfrak{B}_{1}$ and Bautin's system $\mathbf{B}_{\lambda}$ corresponds to a weak center of order $k>0$, then $B_{\lambda}$ is linear. Moreover, a weak center of a quadratic system 
which is not an isochrone has order at most two. Finally, a system in Bautin's form is an isochrone if and only if $\lambda=\left(\lambda_{2}, \lambda_{3}, \lambda_{4}, \lambda_{5}, \lambda_{6}\right)$ lies in one of the sets

$$
\begin{aligned}
& \mathfrak{V}_{1}=\left\{\lambda \mid \lambda_{3}=\lambda_{6}, \lambda_{4}=-4 \lambda_{3}, \lambda_{5}=-4 \lambda_{2}\right\}, \\
& \mathfrak{V}_{2}=\left\{\lambda \mid \lambda_{2}=\lambda_{5}=\lambda_{6}=0, \lambda_{4}=-3 \lambda_{3}\right\}, \\
& \mathfrak{V}_{3}=\left\{\lambda \mid \lambda_{2}=\lambda_{5}=\lambda_{6}=0, \lambda_{4}=-6 \lambda_{3}\right\}, \\
& \mathfrak{V}_{4}=\left\{\lambda \mid \lambda_{2}=\lambda_{5}=0, \lambda_{3}=-4 s, \lambda_{4}=10 s, \lambda_{6}=-s \text { for } s \in \mathbb{R}\right\} .
\end{aligned}
$$

Proof. The analysis is contained in the four cases for a center.

Case 1. If $\lambda_{4}=0$ and $\lambda_{5}=0$, then

$$
p_{2}(\lambda)=\frac{\pi}{6}\left(8 \lambda_{2}^{2}+9 \lambda_{3}^{2}-6 \lambda_{3} \lambda_{6}+5 \lambda_{6}^{2}\right),
$$

and $p_{2}(\lambda)=0$ only when $\lambda_{2}=\lambda_{3}=\lambda_{6}=0$. Thus the system $\mathbf{B}_{\lambda}$ is linear.

Case 2. If $\lambda_{3}=\lambda_{6}$, then

$$
p_{2}(\lambda)=\frac{\pi}{12}\left(\left(4 \lambda_{2}+\lambda_{5}\right)^{2}+\left(4 \lambda_{3}+\lambda_{4}\right)^{2}\right),
$$

and $p_{2}(\lambda)=0$ only when $\lambda_{5}=-4 \lambda_{2}$ and $\lambda_{4}=-4 \lambda_{3}$. In this case $\mathbf{B}_{\lambda}$ has the form

$$
\dot{x}=-y-\lambda_{3} x^{2}-2 \lambda_{2} x y+\lambda_{3} y^{2}, \quad \dot{y}=x+\lambda_{2} x^{2}-2 \lambda_{3} x y-\lambda_{2} y^{2} .
$$

If we choose an angle $\theta$ satisfying $\lambda_{3} \cos \theta+\lambda_{2} \sin \theta=0$, and rotate the system through the angle $\theta$, then we obtain, in the new coordinates, a system of the form

$$
\dot{x}=-y+2 G x y, \quad \dot{y}=x-G x^{2}+G y^{2},
$$

where $G=\left(\lambda_{3} \sin \theta-\lambda_{2} \cos \theta\right)$. If $G \neq 0$, then we can make a second change of variables, namely $u=2 G x$, and $v=2 G y$, to obtain the system

$$
\dot{x}=-y+x y, \quad \dot{y}=x-\frac{1}{2} x^{2}+\frac{1}{2} y^{2},
$$

which we will show below is an isochrone.

Case 3. If $\lambda_{5}=0, \lambda_{4}+5 \lambda_{3}-5 \lambda_{6}=0$, and $\lambda_{3} \lambda_{6}-2 \lambda_{6}^{2}-\lambda_{2}^{2}=0$, then

$$
p_{2}(\lambda)=-\frac{\pi}{6}\left(\lambda_{3}-\lambda_{6}\right)^{2} \text {. }
$$

Thus $p_{2}(\lambda)=0$ only when $\lambda_{3}=\lambda_{6}$ which is the same as Case 2 .

Case 4. If $\lambda_{2}=\lambda_{5}=0$, then, after a rotation of coordinates, $\mathbf{B}_{\lambda}$ can be written in the form

$$
\dot{x}=-y+\left(2 \lambda_{3}+\lambda_{4}\right) x y, \quad \dot{y}=x+\lambda_{6} x^{2}-\lambda_{3} y^{2} .
$$

This is Loud's system $\mathbf{L}$ with $B:=2 \lambda_{3}+\lambda_{4}, D:=\lambda_{6}$, and $F:=-\lambda_{3}$. Note that if $B=2 \lambda_{3}+\lambda_{4}=0$, then

$$
p_{2}(\lambda)=\frac{\pi}{12}\left(10 \lambda_{6}^{2}+5 \lambda_{6} \lambda_{4}+\lambda_{4}^{2}\right)^{2} .
$$

So, $p_{2}(\lambda)=0$ only when $\lambda_{4}=0$ and $\lambda_{6}=0$. This implies the system is linear. 
On the other hand, if $B=2 \lambda_{3}+\lambda_{4} \neq 0$, a change of variables, $u=B x$ and $v=B y$, will transform the Loud system $\mathbf{L}$ to the form

$$
\dot{x}=-y+x y, \quad \dot{y}=x+\frac{D}{B} x^{2}+\frac{F}{B} y^{2} .
$$

Hence, it suffices to consider only the dehomogenized Loud system

$$
\dot{x}=-y+x y, \quad \dot{y}=x+D x^{2}+F y^{2} \text {. }
$$

Next, following Loud [27], one shows by direct integration of the systems that the four choices for the pair $(D, F)$ given by

$$
I_{1}(0,1), \quad I_{2}\left(-\frac{1}{2}, 2\right), \quad I_{3}\left(0, \frac{1}{4}\right), \quad I_{4}\left(-\frac{1}{2}, \frac{1}{2}\right)
$$

are isochrones. Loud shows these are the only isochrones using the theorem of Urabe [45]. We show how to avoid this by direct calculation of the period coefficients in the expansion of $P(\xi, D, F)$ at $\xi=0$. The period coefficients $p_{2}, p_{4}, p_{6}$ for the dehomogenized Loud system are given in the Quadratic Period Coefficient Lemma. We note that $\operatorname{deg} p_{k}=k$ for $k=2,4,6$. Now by Bezout's theorem, $p_{2}$ and $p_{4}$ have at most eight common zeros counted up to multiplicity over pairs of complex numbers. However, direct substitution shows that the four isochronal points $I_{k}, k=1,2,3,4$, and the three Loud points

$$
\begin{gathered}
L_{1}\left(-\frac{3}{2}, \frac{5}{2}\right), \quad L_{2}\left(\frac{-11+\sqrt{105}}{20}, \frac{15-\sqrt{105}}{20}\right), \\
L_{3}\left(\frac{-11-\sqrt{105}}{20}, \frac{15+\sqrt{105}}{20}\right)
\end{gathered}
$$

are all zeros of $p_{2}$ and $p_{4}$ with $I_{4}$ having multiplicity two (i.e., $p_{2}$ and $p_{4}$ are tangent at $\left.I_{4}\right)$. Thus, these are the only zeros of $p_{2}$ and $p_{4}$ over the field of complex numbers. Now one can check by direct substitution that $p_{6}$ vanishes at $I_{k}, k=1,2,3,4$, but does not vanish at $L_{k}, k=1,2,3$. Thus $I_{k}$, $k=1,2,3,4$, are the only possible isochrones, and $L_{1}, L_{2}, L_{3}$ are the only points where the Loud system has a weak center of order two. Since we have shown that any quadratic system which has $p_{2}=0$ can be transformed into Loud's system by a linear change of coordinates, it is clear that any quadratic system having a weak center of finite order has a weak center of order at most two.

Finally, we note that in the fourth case, where $B:=2 \lambda_{3}+\lambda_{4} \neq 0$, we have $D=\lambda_{6} /\left(2 \lambda_{3}+\lambda_{4}\right)$ and $F=-\lambda_{3} /\left(2 \lambda_{3}+\lambda_{4}\right)$, so when the results in all four cases are combined, we obtain all isochrones for Bautin's system $\mathbf{B}_{\lambda}$, viz., $\lambda$ is in one of the sets $\mathfrak{V}_{i}, i=1, \ldots, 4$.

In view of Loud's Theorem and the Finite Order Bifurcation Lemma in $\S 2$, we note that part (i) of the Quadratic Weak Center Bifurcation Theorem has now been proved.

Next we turn to the main problem in this section on quadratic systems, namely, the bifurcation of critical periods from an isochrone, and give the proof 
of part (ii) of the Quadratic Weak Center Bifurcation Theorem. First we recall that from Loud's Theorem the cone ISOV $:=\bigcup_{i=1}^{4} \mathfrak{V}_{i}$ is the set of isochrones in the $\lambda$-space. Now we proceed with the proof by examining the Bautin systems $\mathbf{B}_{\lambda}$ in each of the four cases: $\lambda_{*} \in \operatorname{ISOV} \cap \mathfrak{B}_{i}$ and $\lambda:=\lambda_{*}+\delta \lambda \in \mathfrak{B}_{i}$ for $i=1,2,3,4$.

Remark. Let $\lambda_{*}$ denote a point in the variety ISOV which lies on one of the lines $\mathfrak{V}_{i}, i=2,3,4$, and which corresponds to a nonlinear isochrone, i.e., $\lambda_{*} \neq 0$. If we consider a perturbation of $\lambda_{*}$ of the form $\lambda=\lambda_{*}+\delta \lambda$, which remains in the Bautin variety $\mathbf{B V}$, the variety of the ideal $\left(v_{3}, v_{5}, v_{7}\right)$, then it is easy to verify that the perturbation corresponds to a system in the family

$$
\dot{x}=-y-\lambda_{3} x^{2}+\lambda_{6} y^{2}, \quad \dot{y}=x+\left(2 \lambda_{3}+\lambda_{4}\right) x y .
$$

For example, suppose $\lambda_{*} \neq 0$ lies in $\mathfrak{V}_{2}$. One checks that $\mathfrak{V}_{2} \subset \mathfrak{B}_{2}$ meets $\mathfrak{B}_{1}, \mathfrak{B}_{3}$ and $\mathfrak{B}_{4}$ only at the origin. Thus $\lambda_{*} \notin \mathfrak{B}_{1} \cup \mathfrak{B}_{3} \cup \mathfrak{B}_{4}$. Since this union of three closed sets is closed, it follows that there is a neighborhood of $\lambda_{*}$ in BV not contained in the union. If, on the other hand, $\lambda_{*} \in \mathfrak{V}_{1}$ then all perturbations $\lambda=\lambda_{*}+\delta \lambda$ lie in the family of systems given by

$$
\begin{aligned}
& \dot{x}=-y-\lambda_{3} x^{2}+\left(2 \lambda_{2}+\lambda_{5}\right) x y+\lambda_{3} y^{2}, \\
& \dot{y}=x+\lambda_{2} x^{2}+\left(2 \lambda_{3}+\lambda_{4}\right) x y-\lambda_{2} y^{2} .
\end{aligned}
$$

However, since all the subvarieties of BV meet at the origin, for the bifurcation structure near the linear isochrone we must consider perturbations from $\lambda_{*}=0$ which lie in each of the sets $\mathfrak{B}_{i}, i=1, \ldots, 4$.

The first case considered is when $\lambda_{*} \in \operatorname{ISOV} \cap \mathfrak{B}_{1}$ and its perturbations $\lambda=\lambda_{*}+\delta \lambda$ are in $\mathfrak{B}_{1}$. We note that $\lambda_{*} \in \operatorname{ISOV} \cap \mathfrak{B}_{1}$ implies $\lambda_{*}=\mathbf{0}$. Any perturbation of the linear isochrone which lies in $\mathfrak{B}_{1}$ is a center of a Hamiltonian system, and is quite easy to analyze. The result is in the following theorem.

Theorem 3.3. No critical periods bifurcate from the origin from the linear isochrone in the family

$$
\begin{aligned}
& \dot{x}=-y-\lambda_{3} x^{2}+2 \lambda_{2} x y+\lambda_{6} y^{2}, \\
& \dot{y}=x+\lambda_{2} x^{2}+2 \lambda_{3} x y-\lambda_{2} y^{2} .
\end{aligned}
$$

Proof. From part (i) of the Quadratic Period Coefficient Lemma we compute

$$
p_{2}=\frac{\pi}{6}\left(8 \lambda_{2}^{2}+9 \lambda_{3}^{2}-6 \lambda_{3} \lambda_{6}+5 \lambda_{6}^{2}\right)
$$

which is a positive definite quadratic form in all the parameters. The result follows from the Positive Lemma in $§ 2$.

In order to determine ideal membership conditions for some of the ideals which we need to analyze in this section, we need the next variational lemma. In what follows we will use $D_{\lambda}$ to denote the differential operator $\frac{\partial}{\partial \lambda}$. 
Lemma 3.2 [Variation Lemma]. (i) Let $(x, y) \mapsto(f(x, y), g(x, y)), x, y \in \mathbf{R}$, be an analytic mapping such that the system

$$
\dot{x}=-y+\lambda f(x, y), \quad \dot{y}=x+\lambda g(x, y)
$$

has a center at the origin for all $\lambda \in \mathbf{R}$. Then the period function $P$ for this system satisfies $D_{\lambda} P(\xi, 0)=0$ if and only if

$$
\int_{0}^{2 \pi} g(\xi \cos s, \xi \sin s) \cos s-f(\xi \cos s, \xi \sin s) \sin s d s=0 .
$$

(ii) Let $(x, y) \mapsto(f(x, y), g(x, y)), x, y \in \mathbf{R}$, be an analytic mapping such that the system

$$
\dot{x}=-y+x y+\lambda f(x, y), \quad \dot{y}=x-\frac{1}{2} x^{2}+\frac{1}{2} y^{2}+\lambda g(x, y)
$$

has a center at the origin for all $\lambda \in \mathbf{R}$. Then the period function $\boldsymbol{P}$ for this system satisfies $D_{\lambda} P(\xi, 0)=0$ if and only if

$$
\int_{-\infty}^{\infty} \frac{\left(1-(1-\xi)^{2} w^{2}\right) G(w, \xi)-2(1-\xi) w F(w, \xi)}{\left(1+w^{2}\right)^{2}} d w=0,
$$

where

$$
\begin{aligned}
& F(w, \xi):=f\left(\xi \frac{1-(1-\xi) w^{2}}{1+(1-\xi)^{2} w^{2}}, \xi \frac{(2-\xi) w}{1+(1-\xi)^{2} w^{2}}\right), \\
& G(w, \xi):=g\left(\xi \frac{1-(1-\xi) w^{2}}{1+(1-\xi)^{2} w^{2}}, \xi \frac{(2-\xi) w}{1+(1-\xi)^{2} w^{2}}\right) .
\end{aligned}
$$

Proof. We first make some comments which will be used in the proofs of both parts of the lemma. Of course, as usual, $\xi$ is confined to an open interval of the form $J=(0, \varepsilon)$, where $\varepsilon>0$ is small enough so that all orbits through $(\xi, 0)$ are periodic and enclose the origin. It will again be convenient to express the period function as

$$
P(\xi, \lambda)=2 \pi+\rho(\xi, \lambda) .
$$

Let $t \mapsto(x(t, \xi, \lambda), y(t, \xi, \lambda))$ denote the solution of the differential equation (in part (i) or (ii)) through $(\xi, 0)$. Then for $|\xi|$ sufficiently small we have

$$
y(2 \pi+\rho(\xi, \lambda), \xi, \lambda)=0,
$$

and consequently,

$$
\dot{y}(2 \pi, \xi, 0) D_{\lambda} \rho(\xi, 0)+D_{\lambda} y(2 \pi, \xi, 0)=0 .
$$

By Loud's Theorem, $\lambda=0$ (in either part (i) or part (ii)) corresponds to an isochronous center at the origin, and we can conclude that $\rho(\xi, 0)=0$. We also note that if $|\xi|$ is sufficiently small, then $\dot{y}(2 \pi, \xi, 0)=\dot{y}(0, \xi, 0) \neq 0$ for $\xi \neq 0$, and it follows that $D_{\lambda} \rho(\xi, 0)=0$ exactly when $D_{\lambda} y(2 \pi, \xi, 0)=0$. We let $U(t):=D_{\lambda} x(t, \xi, 0)$, and $V(t):=D_{\lambda} y(t, \xi, 0)$. By using the variational equation for the appropriate system we will show that the equivalences stated in parts (i) and (ii) are equivalent to $V(2 \pi)=D_{\lambda} y(2 \pi, \xi, 0)=0$. 
For the proof of the result in part (i) we take $z(t):=U(t)+i V(t)$, then by using the variational equation corresponding to the nonlinear system in part (i), it is easy to verify that $z$ is the unique solution of the following linear initial value problem:

$$
\dot{z}=i z+f(\xi \cos t, \xi \sin t)+i g(\xi \cos t, \xi \sin t), \quad z(0)=0 .
$$

Using the variation of parameters formula to represent the solution $z$ of this initial value problem, and noting that the imaginary part of $z(t)$ is $V(t)=$ $D_{\lambda} y(t, \xi, 0)$, we find, after some calculation, that $V(2 \pi)=0$ is precisely equivalent to the vanishing of the definite integral in part (i).

For the system in part (ii) the idea of the proof is the same but the computations are more complicated. We simplify the notation by writing $x(t)=$ $x(t, \xi, 0)$ and $y(t)=y(t, \xi, 0)$. One can verify that

$$
x(t)=\frac{\left(2 \xi-\xi^{2}\right) \cos t+\xi^{2}}{\left(2 \xi-\xi^{2}\right) \cos t+\left(2-2 \xi+\xi^{2}\right)}, \quad y(t)=\frac{\left(2 \xi-\xi^{2}\right) \sin t}{\left(2 \xi-\xi^{2}\right) \cos t+\left(2-2 \xi+\xi^{2}\right)}
$$

for $0 \leq \xi<1$. The time dependent coefficient matrix for the variational equations for $U$ and $V$ corresponding to the nonlinear system in part (ii) is once again skew symmetric, so we introduce the complex coordinate $z=U+i V$ as before and find that $z$ is the solution to the initial value problem

$$
\dot{z}(t)=\mathfrak{a}(t) z(t)+\mathfrak{f}(t), \quad z(0)=0,
$$

where $a(t):=y(t)+i(1-x(t))$ and $\mathfrak{f}(t):=f(x(t), y(t))+i g(x(t), y(t))$. If we define

$$
\mathfrak{A}(t):=\int_{0}^{t} \mathfrak{a}(s) d s,
$$

then we must determine when the imaginary part of the expression

$$
z(2 \pi)=\exp (\mathfrak{A}(2 \pi)) \int_{0}^{2 \pi} \exp (-\mathfrak{A}(s)) \mathfrak{f}(s) d s
$$

is zero. If $\xi \neq 1$, then after some elementary integration we get

$$
\mathfrak{A}(t)=2 i \tan ^{-1}((1-\xi) w)-\ln \left|\frac{\left(2 \xi-\xi^{2}\right) \cos t+\left(2-2 \xi+\xi^{2}\right)}{2}\right|,
$$

where $w:=\tan \frac{t}{2}$. We note that $\mathfrak{A}(2 \pi)=0$. Using this fact and the formula for $z(2 \pi)$, we find that the imaginary part of $z(2 \pi)$ is given by

$$
\begin{aligned}
V(2 \pi)=\frac{1}{2} \int_{0}^{2 \pi} & {\left[\left(2 \xi-\xi^{2}\right) \cos t+2-2 \xi+\xi^{2}\right] } \\
& \times\left[f(t) \sin \left(2 \tan ^{-1}((\xi-1) w)\right)+g(t) \cos \left(2 \tan ^{-1}((\xi-1) w)\right)\right] d t,
\end{aligned}
$$

where we have used the abbreviations $f(t):=f(x(t), y(t))$ and $g(t):=$ $g(x(t), y(t))$. Now make the change of variable $w=\tan \frac{t}{2}$ in the preceding integral. If we consider the integral as a sum of the integrals over the intervals 
$[0, \pi]$ and $[\pi, 2 \pi]$, the limits of integration in the transformed variables are $[0, \infty)$ and $(-\infty, 0]$ respectively. In terms of $w$ we have

$$
x(t)=\xi \frac{1-(1-\xi) w^{2}}{1+(1-\xi)^{2} w^{2}}, \quad y(t)=\xi \frac{(2-\xi) w}{1+(1-\xi)^{2} w^{2}},
$$

and thus, after some calculations, we find that

$$
V(2 \pi)=2 \int_{-\infty}^{\infty} \frac{\left(1-(1-\xi)^{2} w^{2}\right) G(w, \xi)-2(1-\xi) w F(w, \xi)}{\left(1+w^{2}\right)^{2}} d w
$$

We turn now to the second case where $\lambda_{*} \in \mathfrak{B}_{2} \cap$ ISOV and its perturbations $\lambda$ are in $\mathfrak{B}_{2}$. In this case we are able to consider bifurcation from the linear isochrone as well as bifurcation from the nonlinear isochrones in $\mathfrak{V}_{1}$ simultaneously. Since $\lambda \in \mathfrak{B}_{2}$, we impose the condition $\lambda_{3}=\lambda_{6}$, and write the corresponding Bautin system in the form

$$
\begin{aligned}
& \dot{x}=-y-\lambda_{3} x^{2}-2 \lambda_{2} x y+\lambda_{3} y^{2}+a x y, \\
& \dot{y}=x+\lambda_{2} x^{2}-2 \lambda_{3} x y-\lambda_{2} y^{2}+b x y,
\end{aligned}
$$

where $a=4 \lambda_{2}+\lambda_{5}$ and $b=4 \lambda_{3}+\lambda_{4}$. By Loud's Theorem this system is isochronous exactly when $a^{2}+b^{2}=0$. The period function $P$ for this system can be expressed as a convergent Taylor series in the form

$$
P\left(\xi, \lambda_{2}, \lambda_{3}, a, b\right)=2 \pi+p_{2}\left(\lambda_{2}, \lambda_{3}, a, b\right) \xi^{2}+p_{3}\left(\lambda_{2}, \lambda_{3}, a, b\right) \xi^{3}+\cdots,
$$

where each $p_{k}, k=2,3, \ldots$, is a polynomial in the parameters $\lambda_{2}, \lambda_{3}, a, b$. Theorem 3.4. The ideal $\mathfrak{m}:=\left(p_{2}, p_{3}, p_{4}, \ldots\right)$ is generated by $p_{2}$ over the polynomial ring $\mathbf{R}\left[\lambda_{2}, \lambda_{3}, a, b\right]$, where $p_{2}\left(\lambda_{2}, \lambda_{3}, a, b\right)=\frac{\pi}{12}\left(a^{2}+b^{2}\right)$.

Proof. We first find a condition equivalent to the ideal membership. Set $a=$ $\sigma \cos \phi$, and $b=\sigma \sin \phi$. Consider $\lambda_{2}, \lambda_{3}, \phi$ as fixed with $\sigma$ as parameter. We abuse notation by writing $\xi \mapsto P(\xi, \sigma)$ for the corresponding period function. We note that $P(\xi, 0) \equiv 2 \pi$ because when $\sigma=0$ the differential system is an isochrone. If $\rho(\xi, \sigma):=P(\xi, \sigma)-2 \pi$, then $\rho(\xi, 0) \equiv 0$, and, of course, $\rho(\xi, \sigma) \not \equiv 0$ for $\sigma \neq 0$. Thus, the Taylor coefficients of $\rho$, namely the $p_{k}$, $k=2,3, \ldots$, will have $\sigma^{2}$ as factor if $\frac{\partial \rho}{\partial \sigma}(\xi, 0) \equiv 0$. Clearly, this is equivalent to each $p_{k}$ having $a^{2}+b^{2}$ as a factor. Thus we proceed to use the Variation Lemma to show $\frac{\partial \rho}{\partial \sigma}(\xi, 0) \equiv 0$.

In order to treat the general case some changes of variables are necessary. Since these variable changes are singular when $\lambda_{2}^{2}+\lambda_{3}^{2}=0$, we examine this case first. If $\lambda_{2}^{2}+\lambda_{3}^{2}=0$ we must consider the system of equations

$$
\dot{x}=-y+x y \sigma \cos \phi, \quad \dot{y}=x+x y \sigma \sin \phi .
$$

But then, an application of part (i) of the Variation Lemma shows our derivative condition is satisfied since

$$
\sigma \xi^{2} \int_{0}^{2 \pi} \sin \phi \sin s \cos ^{2} s-\sin \phi \sin ^{2} s \cos s d s=0
$$


For the case $\lambda_{2}^{2}+\lambda_{3}^{2} \neq 0$, we make the linear change of coordinates given by where $\psi$ is chosen so that

$$
\left[\begin{array}{l}
x \\
y
\end{array}\right]=\frac{1}{2 G}\left[\begin{array}{cc}
\cos \psi & -\sin \psi \\
\sin \psi & \cos \psi
\end{array}\right]\left[\begin{array}{l}
u \\
v
\end{array}\right]
$$

$$
\left[\begin{array}{cc}
\cos \psi & \sin \psi \\
-\sin \psi & \cos \psi
\end{array}\right]\left[\begin{array}{l}
\lambda_{3} \\
\lambda_{2}
\end{array}\right]=\left[\begin{array}{c}
0 \\
-G
\end{array}\right],
$$

and $G=\sqrt{\lambda_{2}^{2}+\lambda_{3}^{2}}$. In the $u, v$ coordinates we consider the period function $Q$ defined along the nonnegative $u$-axis. Then $Q$ is a function of $\xi$ and $\sigma$, again suppressing the dependence on $\lambda_{2}, \lambda_{3}, \phi$, and clearly, with $\xi$ the distance from the origin along the positive $u$-axis, we have $Q(\xi, 0) \equiv 2 \pi, Q(\xi, \sigma) \not \equiv 0$ when $\sigma \neq 0$. Under this coordinate transformation the $u$-axis transforms to the line $y=x \tan \psi$. Consider the section map $x=f(\xi, \sigma)$ which assigns to the point on the ray $x=\frac{\xi}{2 G} \cos \psi, y=\frac{\xi}{2 G} \sin \psi, \xi \geq 0$ the point on the $x$-axis cut by the periodic orbit with initial conditions $\left(\frac{\xi}{2 G} \cos \psi, \frac{\xi}{2 G} \sin \psi\right)$. Then, it is clear that

$$
P(f(\xi, \sigma), \sigma)=Q(\xi, \sigma) .
$$

Now differentiate this equality with respect to $\sigma$ and evaluate at $\sigma=0$ to obtain

$$
P^{\prime}(f(\xi, 0), 0) \frac{\partial f}{\partial \sigma}(\xi, 0)+\frac{\partial P}{\partial \sigma}(f(\xi, 0), 0)=\frac{\partial Q}{\partial \sigma}(\xi, 0) .
$$

Note that $P(\xi, 0) \equiv 2 \pi$ so $P^{\prime}(f(\xi, 0), 0) \equiv 0$ and thus

$$
\frac{\partial P}{\partial \sigma}(f(\xi, 0), 0)=\frac{\partial Q}{\partial \sigma}(\xi, 0) .
$$

In other words it suffices to show $\frac{\partial Q}{\partial \sigma}(\xi, 0)=0$.

If we define $\omega$ and $c$ by

$$
\begin{gathered}
\omega=\sigma / 2 G, \\
c(u, v, \psi)=\left(\frac{1}{2} \sin 2 \psi\right) u^{2}+(\cos 2 \psi) u v-\left(\frac{1}{2} \sin 2 \psi\right) v^{2},
\end{gathered}
$$

then in the $u, v$ coordinates the differential equation has the form

$$
\begin{aligned}
& \dot{u}=-v+u v+\omega \cos (\phi-\psi) c(u, v, \psi), \\
& \dot{v}=u-\frac{1}{2} u^{2}+\frac{1}{2} v^{2}+\omega \sin (\phi-\psi) c(u, v, \psi) .
\end{aligned}
$$

In these variables we have the period function

$$
Q(\xi, \omega)=2 \pi+\tau(\xi, \omega),
$$

and it suffices to show $\frac{\partial Q}{\partial \omega}(\xi, 0)=0$.

But again, by the Variation Lemma, this is equivalent to the vanishing of the integral

$$
\begin{aligned}
\mathfrak{I}:=\frac{\xi^{2}}{2} \int_{-\infty}^{\infty} & \left(\left(1-(1-\xi) w^{2}\right) \cos \psi-(2-\xi) w \sin \psi\right) \\
& \times\left(\left(1-(1-\xi) w^{2}\right) \sin \psi+(2-\xi) w \cos \psi\right) \\
& \times \frac{\left(\left(1-(1-\xi)^{2} w^{2}\right) \sin (\phi-\psi)+2(1-\xi) w \cos (\phi-\psi)\right)}{\left(1+w^{2}\right)^{2}\left(1+(1-\xi)^{2} w^{2}\right)^{2}} d w .
\end{aligned}
$$


The integrand is a rational function $w \mapsto \Psi(w, \xi)$, where the denominator has degree eight, and the degree of the numerator does not exceed six. The numerator and denominator of $\Psi$ do not have any zeros in common for $\xi>0$ and sufficiently small. Thus $\Psi(w, \xi)$ has poles of order two at $w= \pm i$ and $w= \pm i /(1-\xi)$ for all sufficiently small $\xi \in(0,1)$. It follows that the integral $\mathfrak{I}$ is $2 \pi i$ times the sum of the residues of $w \mapsto \Psi(w, \xi)$, in the upper half-plane. A calculation will show that the sum of the two residues in the upper half-plane is zero, and thus $\mathfrak{I}=0$, as claimed.

Corollary 3.1. No critical periods bifurcate from the origin for the isochrones of the form

$$
\begin{aligned}
& \dot{x}=-y-\lambda_{3} x^{2}-2 \lambda_{2} x y+\lambda_{3} y^{2}, \\
& \dot{y}=x+\lambda_{2} x^{2}-2 \lambda_{3} x y-\lambda_{2} y^{2} .
\end{aligned}
$$

Remark. We conjecture the stronger result that in case $\lambda_{3}=\lambda_{6}$ in the Bautin form and the system is not an isochrone, then the period function is globally monotone increasing. An interesting special case of this conjecture can be shown to be true, via., the period function for the system

$$
\dot{x}=-y+a x y, \quad \dot{y}=x+b x y
$$

with $a^{2}+b^{2} \neq 0$ is globally monotone increasing. This can be verified by using the results in [11, 51].

For the third case we have $\lambda_{*} \in \operatorname{ISOV} \cap \mathfrak{B}_{3}$ and its perturbations $\lambda$ are in $\mathfrak{B}_{3}$. We note that $\lambda_{*} \in \operatorname{ISOV} \cap \mathfrak{B}_{3}$ implies $\lambda_{*}=\mathbf{0}$, and thus once again we have only to examine bifurcations from the linear isochrone. Since any perturbation is contained in one of the sets $\mathfrak{B}_{3}^{ \pm}$we examine the bifurcations from the linear isochrone in the two families corresponding to this decomposition of $\mathfrak{B}_{3}$. Our conclusions are contained in the next theorem.

Theorem 3.5. No critical periods bifurcate from the linear isochrone in either the family

$$
\begin{aligned}
& \dot{x}=-y-\left(\sigma^{2}+2 \tau^{2}\right) x^{2}+2 \sigma \tau x y+\tau^{2} y^{2}, \\
& \dot{y}=x+\sigma \tau x^{2}-\left(3 \sigma^{2}+\tau^{2}\right) x y-\sigma \tau y^{2},
\end{aligned}
$$

or the family

$$
\begin{aligned}
& \dot{x}=-y+\left(\sigma^{2}+2 \tau^{2}\right) x^{2}+2 \sigma \tau x y-\tau^{2} y^{2}, \\
& \dot{y}=x+\sigma \tau x^{2}+\left(3 \sigma^{2}+\tau^{2}\right) x y-\sigma \tau y^{2} .
\end{aligned}
$$

Proof. For each family we compute, from the Quadratic Period Coefficient Lemma, that

$$
p_{2}(\sigma, \tau)=\frac{\pi}{6}\left(\sigma^{2}+\tau^{2}\right)^{2}
$$

Thus the result is an immediate consequence of the Positive Lemma. 
In the final case we have $\lambda_{*} \in \mathfrak{B}_{4} \cap$ ISOV, and its perturbations $\lambda$ are in $\mathfrak{B}_{4}$. This is the most difficult case because $\mathfrak{B}_{4} \cap \mathfrak{V}_{k} \neq \varnothing$ for $k=1,2,3,4$. We change coordinates using $u=y$ and $v=-x$, and obtain

$$
\dot{u}=-v-\left(2 \lambda_{3}+\lambda_{4}\right) u v, \quad \dot{v}=u-\lambda_{6} u^{2}+\lambda_{3} v^{2} .
$$

After we rename the variables we obtain the homogeneous Loud system $\mathbf{L}$,

$$
\dot{x}=-y+B x y, \quad \dot{y}=x+D x^{2}+F y^{2},
$$

for which we will prove the following critical result.

Theorem 3.6. At most one critical period can bifurcate from a nonlinear isochrone in the homogeneous Loud system $\mathbf{L}$. At most two critical periods bifurcate from the linear isochrone in this system. Moreover, there are bifurcations to one critical period from the nonlinear isochrone and bifurcations to two critical periods from the linear isochrone.

The remainder of this section is devoted to the proof of this theorem. First we consider perturbations from the nonlinear isochrones. For these perturbations it suffices to consider the dehomogenized Loud system, i.e., the case $B=1$. To see this, observe, by making the change of variables $u=\alpha x$ and $v=\alpha y$, that the period function remains invariant on (punctured) lines through the origin in $(B, D, F)$ space. Thus, the behavior of the period function in a ball about a nonzero point in the space is completely determined, if the $B$ coordinate is not zero, by its behavior on the disk in the plane given by $B=1$ defined by projection of the ball into the plane along lines through the origin.

Following [53] we use ${ }^{a} p_{k}$ for the dehomogenized polynomials, ${ }^{a} p_{k}(D, F)$ $:=p_{k}(1, D, F)$, where $p_{k}$ are the period coefficients for the homogeneous Loud system $\mathbf{L}$ (cf. the Quadratic Period Coefficient Lemma). We are now ready to state our ideal membership condition for the dehomogenized ideal ${ }^{a} \mathfrak{m}_{2}=\left({ }^{a} p_{2},{ }^{a} p_{4},{ }^{a} p_{6}\right)$ corresponding to the dehomogenized Loud system. The algebraic results required for the proof are given in Appendix A.

Lemma 3.3 [Ideal Membership Lemma]. Let ${ }^{a} p_{k}$, for $k \geq 2$, be the period coefficients for the dehomogenized Loud system, and let $\mathfrak{M}$ denote either of the two ideals

$$
{ }^{a} \mathfrak{m}_{1}=\left({ }^{a} p_{2},{ }^{a} p_{4}\right), \quad{ }^{a} \mathfrak{m}_{2}=\left({ }^{a} p_{2},{ }^{a} p_{4},{ }^{a} p_{6}\right)
$$

in $\mathbf{R}[D, F]$. A polynomial $f \in \mathbf{R}[D, F]$ is in $\mathfrak{M}$ if and only if $f$ vanishes on $V(\mathfrak{M})$ and

$$
\left.\frac{d}{d t} f(t,-t)\right|_{t=-1 / 2}=0 \text {. }
$$

Proof. The polynomials ${ }^{a} p_{2},{ }^{a} p_{4},{ }^{a} p_{6}$ are given in the Quadratic Period Coefficient Lemma. By Loud's Theorem,

$$
V\left({ }^{a} \mathfrak{m}_{2}\right)=\left\{I_{1}, I_{2}, I_{3}, I_{4}\right\}
$$

and

$$
V\left({ }^{a} \mathfrak{m}_{1}\right)=V\left({ }^{a} \mathfrak{m}_{2}\right) \cup\left\{L_{1}, L_{2}, L_{3}\right\}
$$


A direct calculation shows that the Jacobian matrices for the two mappings $(D, F) \mapsto\left({ }^{a} p_{2}(D, F),{ }^{a} p_{4}(D, F),{ }^{a} p_{6}(D, F)\right)$ and $(D, F) \mapsto\left({ }^{a} p_{2}(D, F)\right.$, $\left.{ }^{a} p_{4}(D, F)\right)$ both have rank 2 at the points $I_{1}, I_{2}, I_{3}, L_{1}, L_{2}, L_{3}$. Hence the exponents in the primary decomposition at these points are all one by the Exponent One Lemma in Appendix A. The rows of these Jacobian matrices are all parallel to the vector $(1,1)$ at the point $(D, F)=I_{4}\left(-\frac{1}{2}, \frac{1}{2}\right)$. At this point one can verify that for either ideal $\mathfrak{m}_{1}$ or $\mathfrak{m}_{2}$ the exponent of the primary decomposition at $I_{4}$ is two by using (ii) of the Two Variable Exponent Two Lemma in Appendix A. Hence all conditions of the Two Variable Ideal Membership Corollary in Appendix A are satisfied, and we conclude that $f \in{ }^{a} \mathfrak{m}_{k}$ if and only if $f\left(V\left({ }^{a} \mathfrak{m}_{k}\right)\right)=\{0\}$ and $\nabla f\left(-\frac{1}{2}, \frac{1}{2}\right) \|(1,1)$ for $k=1$ or $k=2$. This latter condition is clearly equivalent to the final condition stated in the Ideal Membership Lemma.

The Ideal Membership Lemma can now be applied to prove the following theorem.

Theorem 3.7. The period coefficients ${ }^{a} p_{k}$ for the dehomogenized Loud system are in the ideal ${ }^{a} \mathfrak{m}_{2}=\left({ }^{a} p_{2},{ }^{a} p_{4},{ }^{a} p_{6}\right)$ for $k \geq 2$.

Proof. By Loud's Theorem each of the four points in the variety $V\left({ }^{a} \mathfrak{m}_{2}\right)$ corresponds to an isochrone. Thus, ${ }^{a} p_{k}(D, F)$ vanishes on $V\left({ }^{a} \mathfrak{m}_{2}\right)$ for $k \geq 2$. So, by the Ideal Membership Lemma, the result will follow as soon as we show

which is equivalent to

$$
\left.\frac{d}{d t} a p_{k}(t,-t)\right|_{t=-1 / 2}=0 \text {, }
$$

$$
\left.\frac{d}{d \lambda}{ }^{a} p_{k}\left(\lambda-\frac{1}{2}, \frac{1}{2}-\lambda\right)\right|_{\lambda=0}=0
$$

Now, with $D:=\lambda-\frac{1}{2}$ and $F:=\frac{1}{2}-\lambda$, we obtain the system

$$
\dot{x}=-y+x y, \quad \dot{y}=x-\frac{1}{2} x^{2}+\frac{1}{2} y^{2}+\lambda\left(x^{2}-y^{2}\right) .
$$

If the period function for this system is $P(\xi, \lambda)$, then the derivative condition in the ideal membership condition is equivalent to the condition that $\lambda$ is a factor of $P(\xi, \lambda)-2 \pi$, i.e., equivalent to

$$
\frac{\partial P}{\partial \lambda}(\xi, 0)=0 .
$$

By the Variation Lemma this is equivalent to the vanishing of the integral

$$
\xi^{2} \int_{-\infty}^{\infty} \frac{\left(1-(1-\xi)^{2} w^{2}\right)\left(1-\left(6-6 \xi+\xi^{2}\right) w^{2}+(1-\xi)^{2} w^{4}\right)}{\left(1+w^{2}\right)^{2}\left(1+(1-\xi)^{2} w^{2}\right)^{2}} d w
$$

The residue calculus can once again be used to show that this integral is zero. We omit the details.

We now have the ideal membership result for the dehomogenized version of Loud's system. It is perhaps worth observing that ideal membership in a 
homogeneous ideal leads immediately to membership in any dehomogenization of the ideal, however, obtaining membership conditions for the homogeneous ideal based on an analysis of a dehomogenization of the ideal is nontrivial, cf. [42, p. $134 ; 53$, p. $179 \mathrm{ff}]$. Nonetheless, we are able to link our analysis of the ideal of all period coefficients of the dehomogenized Loud system to the corresponding homogeneous ideal to obtain the following basic theorem.

Theorem 3.8. The period coefficients $p_{k}$ for the homogeneous Loud system $\mathbf{L}$ are in the ideal $\mathrm{m}_{2}=\left(p_{2}, p_{4}, p_{6}\right)$, in the ring $\mathrm{R}[B, D, F]$, for $k \geq 2$.

Proof. Following [53], we let ${ }^{a_{\mathfrak{m}}}$ denote the dehomogenization of an ideal $\mathfrak{m}$ in $\mathbf{R}[B, D, F]$ using the first indeterminant $B$ and we let ${ }^{h} \mathrm{~m}$ denote the homogenization of an ideal $\mathfrak{m}$ in $\mathbf{R}[D, F]$ using a third indeterminant $B$. In the proof of the Ideal Membership Lemma we in effect calculated the following primary decomposition of ${ }^{a_{\mathfrak{m}_{2}}}$ :

$$
{ }_{\mathfrak{m}_{2}}=\mathfrak{q}_{1} \cap \mathfrak{q}_{2} \cap \mathfrak{q}_{3} \cap \mathfrak{q}_{4}
$$

where

$$
\begin{aligned}
& \mathfrak{q}_{1}=\left({ }^{a} \mathfrak{m}_{2}, D, F-1\right)=(D, F-1), \\
& \mathfrak{q}_{2}=\left({ }^{a} \mathfrak{m}_{2}, D, F-\frac{1}{4}\right)=(D, 4 F-1), \\
& \mathfrak{q}_{3}=\left({ }^{a} \mathfrak{m}_{2}, D+\frac{1}{2}, F-2\right)=(2 D+1, F-2), \\
& \mathfrak{q}_{4}=\left({ }^{a}{ }{ }_{2},\left(D+\frac{1}{2}\right)^{2},\left(F-\frac{1}{2}\right)^{2},\left(D+\frac{1}{2}\right)\left(F-\frac{1}{2}\right)\right)=\left(D+F,(2 D+1)^{2}\right) .
\end{aligned}
$$

It follows that

$$
\mathfrak{m}_{2} \subset{ }^{h}\left({ }^{a} \mathfrak{m}_{2}\right)={ }^{h} \mathfrak{q}_{1} \cap{ }^{h} \mathfrak{q}_{2} \cap{ }^{h} \mathfrak{q}_{3} \cap{ }^{h} \mathfrak{q}_{4}
$$

and

$$
\begin{aligned}
& { }^{h} q_{1}=(D, B-F), \quad{ }^{h} q_{2}=(2 B-F, 2 D+B), \\
& { }^{h} \mathfrak{q}_{3}=(D, B-4 F), \quad{ }^{h} q_{4}=\left(D+F,(2 D+B)^{2}\right) \text {. }
\end{aligned}
$$

Since ${ }^{h} \mathfrak{q}_{i}, i=1,2,3,4$, are each generated by two elements with no nonconstant common divisor, it follows from [49, pp. 64-65] that a homogeneous polynomial $f(B, D, F)$ in $\mathbf{R}[B, D, F]$ is in the ideal ${ }^{h}\left({ }^{a} \mathrm{~m}_{2}\right)$ if and only if $f(1, D, F)$ is in ${ }^{a} \mathfrak{m}_{2}$. Thus $p_{k} \in{ }^{h}\left({ }^{a} \mathfrak{m}_{2}\right)$ for $k \geq 2$. Now we define $\mathfrak{i}_{0}=\left(\mathfrak{m}_{2}, B^{4}\right)$. One can verify with the help of the Gröbner basis package in the MACSYMA computer algebra system that

$$
\mathfrak{m}_{2}={ }^{h} \mathfrak{q}_{1} \cap{ }^{h} \mathfrak{q}_{2} \cap{ }^{h} \mathfrak{q}_{3} \cap{ }^{h} \mathfrak{q}_{4} \cap \mathfrak{i}_{0}={ }^{h}\left({ }^{a} \mathfrak{m}_{2}\right) \cap \mathfrak{i}_{0} .
$$

We conclude the proof by verifying that $p_{k} \in \mathfrak{i}_{0}$ for $k \geq 2$. If $2 \leq k \leq 6$, this follows from the definition of $\mathfrak{i}_{0}$ and the Period Coefficient Lemma in $\S 2$. It is easy to show that $V\left(\mathfrak{i}_{0}\right)=\{0\}$. Thus by the Hilbert Nullstellensatz every monomial of sufficiently high degree is in $i_{0}$. With the aid of the computer algebra package mentioned above one can verify that every monomial of degree seven is in $\mathfrak{i}_{0}$. Thus $\mathfrak{i}_{0}$ contains every homogeneous polynomial $f \in \mathbf{R}[B, D, F]$ 
of degree at least seven, and so the period coefficients $p_{k} \in \mathfrak{i}_{0}$ for $k \geq 7$, by part (i) of the Quadratic Period Coefficient Lemma.

We now know that at most two critical periods bifurcate from an isochrone in Loud's system. To complete the proof of the First Quadratic Isochrone Bifurcation Theorem we prove a local ideal membership theorem for the dehomogenized Loud system which together with the Isochrone Bifurcation Theorem will show at most one critical period bifurcates from a nonlinear isochrone.

Theorem 3.9. The period coefficients ${ }^{a} p_{k}, k \geq 2$, for the dehomogenized Loud system, are in the ideal $\left({ }^{a} p_{2},{ }^{a} p_{4}\right)$ in the local ring $\mathbf{R}\{D, F\}_{\lambda_{*}}$ localized at any one of the isochrones $\lambda_{*}:=(D, F)$ in the set

$$
\left\{I_{1}(0,1), I_{2}\left(-\frac{1}{2}, 2\right), I_{3}\left(0, \frac{1}{4}\right), I_{4}\left(-\frac{1}{2}, \frac{1}{2}\right)\right\} \text {. }
$$

Moreover, ${ }^{a} p_{2}$ is independent with respect to ${ }^{a} p_{4}$ at each isochrone.

Proof. We construct a polynomial $f \in \mathbf{R}[D, F]$ such that $f\left(\lambda_{*}\right) \neq 0$ for each point $\lambda_{*}$ corresponding to an isochrone, and such that $f^{a} p_{6} \in{ }_{\mathfrak{m}}=\left({ }^{a} p_{2},{ }^{a} p_{4}\right)$. It is easy to construct such an $f$ by using the Ideal Membership Lemma. For example,

$f(D, F):=\left(c_{1} D+c_{2} F+c_{3}\right)(2 D+3)\left(400 D^{2}+440 D+16\right)\left(400 F^{2}-600 F+120\right)$

vanishes at each of the Loud points $L_{1}, L_{2}, L_{3}$. Moreover, we can choose constants $c_{1}, c_{2}, c_{3}$ so that $f$ does not vanish at $I_{1}, I_{2}, I_{3}, I_{4}$, and so that the gradient of $f^{a} p_{6}$ is parallel to $(1,1)$ at $I_{4}$. One choice for the constants is

$$
c_{1}=0, \quad c_{2}=-1 / 4096, \quad c_{3}=9 / 40960 .
$$

Now, for ${ }^{a} p_{k}, k \geq 2$, we already know

$$
{ }^{a} p_{k}=\alpha^{a} p_{2}+\beta^{a} p_{4}+\gamma^{a} p_{6}
$$

where $\alpha, \beta, \gamma \in \mathbf{R}[D, F]$. Since $f^{a} p_{6} \in\left({ }^{a} p_{2},{ }^{a} p_{4}\right)$, one easily verifies that $f^{a} p_{k} \in\left({ }^{a} p_{2},{ }^{a} p_{4}\right)$ for $k \geq 2$. Whence ${ }^{a} p_{k} \in \mathbf{R}\{D, F\}_{\lambda_{*}}$ for $k \geq 2$.

Finally, one can check that ${ }^{a} p_{2}$ is independent with respect to ${ }^{a} p_{4}$ at each isochrone.

\section{SECOND ORDER CONSERVATIVE SYSTEMS}

In this section we examine the bifurcation of critical periods for conservative second order scalar differential equations of the form

$$
\ddot{u}+g(u)=0 \text {. }
$$

The potential energy $V(u)$ for this differential equation is defined by

$$
V(u):=\int_{0}^{u} g(s) d s,
$$

and the total energy is given by the Hamiltonian

$$
H(u, v):=\frac{1}{2} v^{2}+V(u), \quad \text { where } v=\dot{u} .
$$


We always assume there is a center at the origin and the linearization at the origin is normalized to $\ddot{u}+u=0$. Our analysis of the bifurcation problem requires a convenient characterization of the period coefficients $p_{k}$ and a knowledge of the isochrones. The needed information is contained in the next lemma and the theorem which follows.

Lemma 4.1 [Potential Period Lemma]. Let the potential function $V$ be analytic on $\mathbf{R}, V^{\prime}(0)=0$, and $V^{\prime \prime}(0)=1$. The following statements are true:

(i) The differential equation $\ddot{u}+g(u)$ has a linear center at the origin, and there are periodic orbits with energies $E=V(u)$ up to the first positive critical value of $V$.

(ii) If $X:=h(u):=\operatorname{sgn} u \sqrt{2 V(u)}$, when $V(u) \geq 0$, then $h$ is analytic on the connected component of $\{u \mid V(u) \geq 0\}$ which contains 0 . The inverse function $X \mapsto h^{-1}(X)$ is defined and analytic on the connected component of $\left\{u \mid h^{\prime}(u)>0\right\}$, which contains 0 , and $\left(h^{-1}\right)^{\prime}(0)=1$.

(iii) For all positive energies $E=V(u)$ up to the first positive critical value of $V$, the period function $E \mapsto P(E)$ is given by

$$
P(E)=2 \int_{-\pi / 2}^{\pi / 2} \frac{1}{h^{\prime}\left(h^{-1}(\sqrt{2 E} \sin \theta)\right)} d \theta .
$$

(iv) If the MacLaurin series for $h^{-1}$ is given by

$$
h^{-1}(X)=X+\sum_{k=2}^{\infty} d_{k} X^{k}
$$

then the period function $X \mapsto P(X)$ is given by $P(X)=2 \pi+\sum_{k=1}^{\infty} p_{2 k} X^{2 k}$, where

$$
p_{2 k}=2 \pi(2 k+1) \frac{1 \cdot 3 \cdot 5 \cdots(2 k-1)}{2 \cdot 4 \cdot 6 \cdots 2 k} d_{2 k+1}, \quad k \geq 1 .
$$

(v) If $V^{\prime}(u) \neq 0$ for $u \neq 0$, and if

$$
\lim _{u \rightarrow \pm \infty} \operatorname{sgn} u \frac{V^{\prime}(u)}{\sqrt{2 V(u)}}=\infty
$$

then $\lim _{E \rightarrow \infty} P(E)=0$.

Proof. Statement (i) is a direct consequence of the discussion in Arnold [2, pp. 87-90].

For statement (ii) we note that since $V$ has a quadratic minimum at 0 , and $V(0)=0$, it follows that $V(u) \geq 0$ on some neighborhood of 0 . Hence $u \mapsto h(u)$ is defined and analytic on the connected component of $\{u \mid V(u) \geq 0\}$ which contains 0 . Since $h^{\prime}(0)=1$, one can show that $X \mapsto h^{-1}(X)$ is defined and analytic on the connected component of $\left\{u \mid h^{\prime}(u)>0\right\}$, which contains 0 , and $\left(h^{-1}\right)^{\prime}(0)=1$.

Turning now to the result in (iii), we let $U_{0}$ denote the connected component of $\left\{u \mid h^{\prime}(u)>0\right\}$ which contains 0 . Then from (i) it follows that any periodic 
phase trajectory starting at $\left(u_{0}, 0\right)$ with $u_{0} \in U_{0}$ will first cross the $u$-axis again at $\left(u_{1}, 0\right)$, with $u_{1} \in U_{0}$. Let $u_{0} \in U_{0}$. The total energy $E$ is constant along the periodic phase trajectory starting at $\left(u_{0}, 0\right)$, i.e.

$$
E:=\frac{1}{2} v^{2}+V(u)=V\left(u_{0}\right),
$$

and the corresponding period is given by

$$
P(E)=\frac{2}{\sqrt{2}} \int_{u_{0}}^{u_{1}} \frac{1}{\sqrt{E-V(u)}} d u .
$$

Next we make the change of variables $X=h(u)$ which has the effect of transforming the periodic trajectories into circles centered at the origin, and we get

$$
P(E)=\frac{2}{\sqrt{2}} \int_{-\sqrt{2 E}}^{\sqrt{2 E}} \frac{X}{V^{\prime}\left(h^{-1}(X)\right) \sqrt{E-X^{2} / 2}} d X .
$$

A final change of variables $X=\sqrt{2 E} \sin \theta$ gives the result claimed in (iii):

$$
\begin{aligned}
P(E) & =2 \int_{-\pi / 2}^{\pi / 2} \frac{\sqrt{2 E} \sin \theta}{V^{\prime}\left(h^{-1}(\sqrt{2 E} \sin \theta)\right)} d \theta \\
& =2 \int_{-\pi / 2}^{\pi / 2} \frac{1}{h^{\prime}\left(h^{-1}(\sqrt{2 E} \sin \theta)\right)} d \theta=2 \int_{-\pi / 2}^{\pi / 2}\left(h^{-1}\right)^{\prime}(\sqrt{2 E} \sin \theta) d \theta .
\end{aligned}
$$

From the last equality and the Maclaurin series representation of $h^{-1}(X)$, we see that if $E>0$ is sufficiently small, then

$$
P(E)=2 \int_{-\pi / 2}^{\pi / 2} 1+2 d_{2} \sqrt{2 E} \sin \theta+3 d_{3}(\sqrt{2 E} \sin \theta)^{2}+\cdots d \theta .
$$

Also, along the $X$-axis we have

$$
E=V(u)=\frac{1}{2} h(u)^{2}=\frac{1}{2} X^{2},
$$

so $X=\sqrt{2 E}$ and we can represent $P$ as a function of $X$ in the form

$$
\begin{aligned}
P(X) & =2 \pi+2 \sum_{k=1}^{\infty}(2 k+1) d_{2 k+1} \int_{-\pi / 2}^{\pi / 2} \sin ^{2 k} \theta d \theta X^{2 k} \\
& =2 \pi+2 \pi \sum_{k=1}^{\infty}(2 k+1) \frac{1 \cdot 3 \cdot 5 \cdots(2 k-1)}{2 \cdot 4 \cdot 6 \cdots 2 k} d_{2 k+1} X^{2 k} \\
& =2 \pi+\sum_{k=1}^{\infty} d_{2 k+1}^{*} X^{2 k}
\end{aligned}
$$

and this proves (iv).

For statement (v), we observe that the conditions assumed on the potential energy function $V$ imply that every trajectory in the punctured phase plane $\mathbf{R}^{2} \backslash\{\mathbf{0}\}$ is periodic [2, pp. 87-90]. Moreover, the function $u \mapsto h(u)$ is in $C^{l}(\mathbf{R})$, and its derivative is given by

$$
h^{\prime}(u)=\operatorname{sgn} u \frac{V^{\prime}(u)}{\sqrt{2 V(u)}} .
$$


Thus, we have that $h^{\prime}(u)>0$ for all $u \in \mathbb{R}$. Hence, $X \mapsto h^{-1}(X)$ is also in $C^{1}(\mathbf{R})$. Since

$$
\lim _{|u| \rightarrow \infty} h^{\prime}(u)=\infty
$$

$h^{\prime}>0$, and $h^{\prime} \in C(\mathbb{R})$, it follows that there is a constant $m>0$ such that

$$
h^{\prime}(u) \geq m \quad \forall u \in \mathbb{R} .
$$

Using the representation of the period function $E \mapsto P(E)$ in statement (iii), and the Lebesgue dominated convergence theorem, we get that $P(E) \rightarrow 0$ as $E \rightarrow \infty$.

Remark. If one compares the power series for $u=h^{-1}(X)$ and $P(X)$ in part (iv) of the Potential Period Lemma, then one can conclude Urabe's Theorem for analytic systems [45, 46].

The center is isochronous if and only if $d_{2 k+1}=0$ for $k \geq 1$,

i.e. $h^{-1}(X)=X+S(X)$, where $S$ is an even function.

For example, if we take $h^{-1}(X)=X+\frac{1}{2} X^{2}$, we obtain the differential equation $\ddot{u}+1-(1+2 u)^{-1 / 2}=0$, which has an isochronous center at the origin.

Theorem 4.1 [Polynomial Potential Isochrone Theorem]. The second order system $\ddot{u}+g(u, \lambda)=0$, with $\lambda=\left(\lambda_{3}, \lambda_{4}, \ldots, \lambda_{N}\right)$, and potential energy

$$
V(u)=\frac{1}{2} u^{2}+\sum_{i=1}^{N-2} \lambda_{i+2} u^{i+2}
$$

has an isochronous center at the origin if and only if $\lambda=0$.

Proof. There is clearly an isochronous center at the origin if $\lambda=0$. Suppose $\lambda \neq 0$, and that $\ddot{u}+g(u, \lambda)=0$ has an isochronous center at the origin. We will show that this implies that the polynomial $V$ satisfies all conditions needed for the conclusion of part ( $v$ ) of the Potential Period Lemma, and this is contrary to the assumption that the periodic motions have constant period. In order to prove this, we use two well-known facts.

(i) Let $\gamma$ denote a maximal phase curve of the second order differential equation $\ddot{u}+g(u)=0$, and let $p \in \gamma$. If the projection of $\gamma$ to the $u$-axis is contained in a subset $I \subseteq \mathbf{R}$ on which the potential energy $V$ is bounded below, then $t \mapsto \phi_{t}(p)$, the phase trajectory through $p$, can be extended to $(-\infty, \infty)$; cf. [2, pp. 69,86$]$.

(ii) Let $p$ denote a rest point surrounded by a continuous family of periodic orbits of the flow $t \mapsto \phi_{t}$ of a relatively prime, analytic plane vector field. If the point set consisting of all periodic points in the family of periodic orbits contains boundary points not equal to $p$, then there is a boundary point which is neither a rest point nor a periodic point of the flow [31].

Let $\Sigma$ denote the periodic points in the continuous family of periodic orbits surrounding the origin in the phase plane. We also consider the projection $I$ 
of $\Sigma$ to the $u$-axis. Let $D(r)$ denote the closed disk centered at the origin of the phase plane with radius $r$. We claim there is some periodic orbit contained in $\Sigma$ which lies in the complement of $D(r)$. If this is not the case, then $\Sigma$ has a boundary point in $D(r) \backslash\{\boldsymbol{0}\}$. By (ii) there is a boundary point $q$ (not necessarily in $D(r)$ ) which is neither a rest point nor a periodic point of the phase flow. From the form of the potential energy we see $V$ is positive and concave upward in a punctured neighborhood of the origin of the $u$-axis. If $V(u)<0$ for some $u \in I$, there is a point $u_{c}$ between $u$ and the origin where $V^{\prime}\left(u_{c}\right)=0$. But then $\left(u_{c}, 0\right)$ lies on a periodic orbit in $\Sigma$. Since no periodic orbit contains a rest point this is a contradiction and we have $V(u) \geq 0$ for all $u$ in the closure of $I$. Since $q$ is in the closure of $\Sigma$, it follows that the projection of the maximal phase curve through $q$ is contained in the closure of $I$. Hence by (i) the solution through $q$ can be extended for all time. Let $T$ denote the common period of the periodic orbits in $\Sigma$ and consider the points $\phi_{-T}(q)$ and $\phi_{T}(q)$. Since $q$ is not periodic and not a rest point these points are distinct. There are disjoint neighborhoods $U_{-T}$ of $\phi_{-T}(q)$ and $U_{T}$ of $\phi_{T}(q)$ such that the minimum time required for a point in $U_{-T}$ to reach $U_{T}$ is larger than $T$. But $q$ is a boundary point of $\Sigma$ so there is a periodic point $z \in \Sigma \cap U_{-T}$ whose orbit meets $U_{T}$. Since $z$ is periodic with period $T$ the minimum time required to meet $U_{T}$ is clearly less than $T$. This contradicts the existence of $q$. Thus, for every $D(r)$ there is a periodic orbit contained in $\Sigma$ which lies in the complement of the disk. This implies the periodic orbits in $\Sigma$ fill the punctured plane. It follows that the origin is the only rest point of the phase flow and therefore $V^{\prime}(u) \neq 0$ for all $u \neq 0$. The only polynomials of the given form with this property are of even degree with positive leading coefficient, i.e.,

$$
V(u)=\frac{1}{2} u^{2}+\lambda_{3} u^{3}+\lambda_{4} u^{4}+\cdots+\lambda_{2 n} u^{2 n}, \quad \lambda_{2 n}>0, n \geq 2 .
$$

Thus all requirements for the conclusion of part $(v)$ of the Potential Period Lemma are satisfied.

Remark. It follows immediately from the Polynomial Potential Isochrone Theorem that if $G(u)$ is a nonzero polynomial with $G(0)=G^{\prime}(0)=0$, then the system

$$
\dot{u}=v, \quad \dot{v}=-u+G(u)
$$

cannot be linearized by a smooth coordinate transformation.

Now before we turn to our analysis of the bifurcation of the critical periods for polynomial potential functions we need to develop some basic facts about ideals which are generated by various operations with infinite series.

Lemma 4.2. Let $A(x)=a_{1} x+a_{2} x^{2}+a_{3} x^{3}+\cdots$ and $B(x)=b_{1} x+b_{2} x^{2}+$ $b_{3} x^{3}+\cdots$ be two formal power series with $a_{i}, b_{i} \in \mathbb{R}\left[\lambda_{1}, \lambda_{2}, \ldots, \lambda_{N}\right]$ for $i=$ $1,2,3, \ldots$. If

$$
C(x):=A(B(x))=c_{1} x+c_{2} x^{2}+c_{3} x^{3}+\cdots,
$$


then $\left\{c_{1}, c_{2}, \ldots, c_{\mu}\right\} \subset\left(a_{1}, a_{2}, \ldots, a_{\mu}\right)$ for $\mu=1,2,3, \ldots$.

Proof. Let

$$
A(x)^{k}=\sum_{n=k}^{\infty} a_{n}^{(k)} x^{n}, \quad k=1,2,3, \ldots,
$$

and

$$
B(x)^{k}=\sum_{n=k}^{\infty} b_{n}^{(k)} x^{n}, \quad k=1,2,3, \ldots
$$

Then [48, Lemma 7.1b, p. 46]

$$
c_{n}=\sum_{k=1}^{n} a_{k} b_{n}^{(k)}
$$

for $n=1,2,3, \ldots$. Since $b_{n}^{(k)} \in \mathbf{R}\left[\lambda_{1}, \lambda_{2}, \ldots, \lambda_{N}\right]$, it follows that $c_{n} \in$ $\left(a_{1}, a_{2}, \ldots, a_{n}\right)$.

Proposition 4.1. Let $A(x)=a_{1} x+a_{2} x^{2}+a_{3} x^{3}+\cdots$ and $B(x)=b_{1} x+b_{2} x^{2}+$ $b_{3} x^{3}+\cdots$ be two formal power series with $a_{i}, b_{i} \in \mathbf{R}\left[\lambda_{1}, \lambda_{2}, \ldots, \lambda_{N}\right]$ for $i=1,2,3, \ldots$ Let $b_{1} \neq 0$, and let all of the coefficients in the power series expansion for the inverse function $B^{-1}(x)$ be in $\mathrm{R}\left[\lambda_{1}, \lambda_{2}, \cdots, \lambda_{N}\right]$. If

$$
C(x):=A(B(x))=c_{1} x+c_{2} x^{2}+c_{3} x^{3}+\cdots,
$$

then $\left(c_{1}, c_{2}, \ldots, c_{\mu}\right)=\left(a_{1}, a_{2}, \ldots, a_{\mu}\right)$ for $\mu=1,2,3, \ldots$.

Proof. The inclusion $\left(c_{1}, c_{2}, \ldots, c_{\mu}\right) \subset\left(a_{1}, a_{2}, \ldots, a_{\mu}\right)$ follows from the preceding lemma. If this lemma is applied again with $A(x)$ replaced by $C(x)$ and $B(x)$ replaced by $B^{-1}(x)$, then we obtain the reverse inclusion $\left(a_{1}, a_{2}, \ldots, a_{\mu}\right)$ $C\left(c_{1}, c_{2}, \ldots, c_{\mu}\right)$.

Lemma 4.3 [Series Reversion Lemma]. Let $f: \mathbf{R} \rightarrow \mathbf{R}$ be analytic at 0 , and $f(0)=1$. Let $p \in \mathbf{R}[t]$ have the form

$$
p(t)=p_{1} t+p_{2} t^{2}+\cdots+p_{n} t^{n} .
$$

Then the function $t \mapsto z:=t f(p(t)), t \in \mathbb{R}$, and its inverse $z \mapsto t:=F(z)$ are analytic at 0 . Moreover, the power series expansion for $F(z)$ has the form

$$
F(z)=z+F_{2} z^{2}+F_{3} z^{3}+\cdots,
$$

where $F_{k} \in \mathbf{R}\left[p_{1}, p_{2}, \ldots, p_{n}\right]$.

Proof. We note that

$$
t f(p(t))=t+v_{2} t^{2}+v_{3} t^{3}+\cdots,
$$

where $v_{k} \in \mathbf{R}\left[p_{1}, p_{2}, \ldots, p_{n}\right]$ for $k=2,3, \ldots$. Since $f(0) \neq 0$, it follows that the inverse function $F$ exists and is analytic in some neighborhood of 0 . The statement that the $F_{k} \in \mathbf{R}\left[p_{1}, p_{2}, \ldots, p_{n}\right]$ for $k \geq 2$, can be seen in several simple ways. However, applications of the results require us to be 
able to efficiently generate the $F_{k}$. Thus we give an algorithm for generating the $F_{k}$, from which it trivially follows that the $F_{k} \in \mathbf{R}\left[p_{1}, p_{2}, \ldots, p_{n}\right]$. This algorithm is an easy consequence of results described in Henrici [25] (cf. the J. C. P. Miller Formula, p. 42, and the Schur-Jabotinski Theorem, p. 55; see also [26, pp. 508-509]). The J. C. P. Miller formula is used to calculate the coefficients $u_{k}$ in the expansion

$$
f(p(t))^{-n}=1+u_{1} t+u_{2} t^{2}+\cdots
$$

for $n=2,3, \ldots$, and the Schur-Jabotinski Theorem is used to get

$$
F_{k}=u_{k-1} / k \text {. }
$$

These result can be organized to produce the following algorithm. The remainder of the proof follows from this result.

Lagrange-Henrici Series Reversion Algorithm.

Input: $n_{\max }$ and $v_{k}$, for $k=2,3, \ldots, n_{\max }$, and $u_{0}=1$.

Output: $F_{k}$, for $k=2,3, \ldots, n_{\max }$.

for $n=2$ until $n_{\max }$ step 1 do

begin

for $m=1$ until $n-1$ step 1 do

$$
u_{m}:=\frac{1}{m} \sum_{k=1}^{m}[(1-n) k-m] u_{m-k} v_{k+1} ;
$$

$$
F_{n}:=\frac{1}{n} u_{n-1}
$$

end.

Theorem 4.2 [Reversion Ideal Theorem]. Let $f: \mathbf{R} \rightarrow \mathbf{R}$ be analytic at 0 , and $f(0)=1$. Let $p \in \mathbf{R}[t]$ have the form

$$
p(t)=p_{1} t+p_{2} t^{2}+\cdots+p_{n} t^{n}
$$

Let the inverse function of $t \mapsto z:=t f(p(t)), t \in \mathbf{R}$, denoted by $z \mapsto t:=F(z)$, have the power series expansion

$$
F(z)=z+F_{2} z^{2}+F_{3} z^{3}+\cdots .
$$

Let $\mathrm{m}$ and $\mathrm{m}_{k}$ denote the ideals $\left(F_{2}, F_{3}, \ldots\right)$ and $\left(F_{2}, F_{3}, \ldots, F_{k+1}\right)$, respectively, in $\mathbf{R}\left[p_{1}, p_{2}, \ldots, p_{n}\right]$ for $k=1,2,3, \ldots$, and let $f^{\prime}(0) \neq 0$. Then $n$, the number of parameters in the polynomial $p(t)$, is the first positive integer $k$ such that $\mathfrak{m}_{k}=\mathfrak{m}$ and $\mathfrak{m}_{n}=\left(p_{1}, p_{2}, \ldots, p_{n}\right)$.

Proof. We use the Lagrange-Bürmann Theorem [25, p. 58] to write

$$
F_{k+1}=\left.\frac{1}{(k+1) !} \frac{d^{k}}{d t^{k}} f(p(t))^{-(k+1)}\right|_{t=0} \text {. }
$$

We calculate that

$$
\frac{\partial F_{k+1}}{\partial p_{i}}=-\left.\frac{1}{k !} \frac{\partial^{k}}{\partial t^{k}} f(p(t))^{-(k+2)} f^{\prime}(p(t)) t^{i}\right|_{t=0}
$$


and thus if $\mathbf{p} \in \mathbb{R}^{n}$ denotes the point with coordinates $p_{i}, i=1,2, \ldots, n$, then we obtain

$$
\left.\frac{\partial F_{k+1}}{\partial p_{i}}\right|_{\mathbf{p}=\mathbf{0}}=-\left.\frac{1}{k !} \frac{\partial^{k}}{\partial t^{k}} f^{\prime}(0) t^{i}\right|_{t=0}=-f^{\prime}(0) \delta_{i, k}
$$

for $k=1,2,3, \ldots$ and $i=1,2, \ldots, n$, where $\delta_{i, k}$ is the Kronecker $\delta$. Thus we find that the Jacobian of the transformation

$$
\mathbf{p} \mapsto\left(F_{2}(\mathbf{p}), F_{3}(\mathbf{p}), \ldots, F_{n+1}(\mathbf{p})\right), \quad \mathbf{p} \in \mathbb{R}^{n},
$$

is $-f^{\prime}(0) \mathbf{I}_{n}$, where $\mathbf{I}_{n}$ is the $n \times n$ identity matrix. One may easily verify that the $v_{k}$ in the power series expansion of $t \mapsto t f(t)$ satisfy

$$
v_{k+1}=f^{\prime}(0) p_{k}+\phi_{k}\left(p_{1}, p_{2}, \ldots, p_{k-1}\right)
$$

for $k=1,2, \ldots, n$, where $\phi_{k} \in \mathfrak{p}_{0}^{2}$. It follows from the Lagrange-Henrici Series Reversion Algorithm that

$$
F_{k+1}\left(p_{1}, p_{2}, \ldots, p_{n}\right)=-f^{\prime}(0) p_{k}+R_{k}\left(p_{1}, p_{2}, \ldots, p_{k-1}\right)
$$

for $k=2,3, \ldots, n+1$, where $R_{k} \in \mathfrak{p}_{0}^{2}$, and since $f^{\prime}(0) \neq 0$, it follows that not all of the $F_{i}$ can belong to $\mathfrak{m}_{k}$ until $k$ is at least $n$. Moreover, it is clear that the equations

$$
F_{k+1}\left(p_{1}, p_{2}, \ldots, p_{n}\right)=0
$$

can be solved in the order $k=1,2, \ldots, n$ by the elimination method to verify that $V(\mathfrak{m})=\{\boldsymbol{0}\}$. The Exponent One Lemma in Appendix $\mathrm{A}$ can now be applied to verify that $\mathfrak{m}_{n}$ is a radical ideal.

The following is a useful fact about reciprocal series, which can be used to connect the ideal generated by the period coefficients with the ideal generated by the scale function in the normal form calculations mentioned in the introduction.

Proposition 4.2. Let $t \mapsto f(t)$ be analytic at 0 and let $f(0)=1$ with

$$
f(t)=1+x_{1} t+x_{2} t^{2}+x_{3} t^{3}+\cdots .
$$

Then

$$
1 / f(t)=1+p_{1} t+p_{2} t^{2}+p_{3} t^{3}+\cdots,
$$

where $p_{k} \in \mathbb{R}\left[x_{1}, x_{2}, \ldots, x_{k}\right]$, and

$$
\left(x_{1}, x_{2}, \ldots, x_{k}\right)=\left(p_{1}, p_{2}, \ldots, p_{k}\right) \text { in } \mathbb{R}\left[x_{1}, x_{2}, \ldots, x_{k}\right]
$$

for $k \geq 1$.

Proof. The polynomials $p_{k}$ are given by the recursion

$$
p_{0}=1, \quad p_{k}=-\sum_{i=1}^{k} p_{k-i} x_{i} .
$$


It follows at once that the variety of $\left(p_{1}, p_{2}, \ldots, p_{k}\right)$ is $\mathbf{0}$, and the ideal is radical in $\mathrm{R}\left[x_{1}, x_{2}, \ldots, x_{k}\right]$. Consequently it coincides with $\left(x_{1}, x_{2}, \ldots, x_{k}\right)$.

For the remainder of this section we will consider polynomial potential functions $V$ of the form

$$
V(u)=\frac{1}{2} u^{2}+\sum_{i=1}^{N-2} \lambda_{i+2} u^{i+2} .
$$

We want to use the Potential Period Lemma to obtain a series expansion for the period function in a neighborhood of 0 . Thus we define the function $\phi$ by the equation

$$
\phi(u)=2 \sum_{i=1}^{N-2} \lambda_{i+2} u^{i}
$$

Then, according to the Potential Period Lemma, we need the analytic solution $u=u(X)$ of the equation

$$
X=h(u)=u \sqrt{1+\phi(u)}
$$

for $|X|$ sufficiently small. In fact, the function $t \mapsto f(t):=\sqrt{1+t}$ satisfies the conditions in the Series Reversion Lemma, and the solution is given by

$$
u(X)=X+d_{2} X^{2}+d_{3} X^{3}+d_{4} X^{4}+\cdots .
$$

The coefficients $d_{n}$ can be calculated by Lagrange's formula [26, p. 508]

$$
d_{n}=\left.\frac{1}{n !} \frac{d^{n-1}}{d^{n-1} u}(1+\phi(u))^{-n / 2}\right|_{u=0}
$$

for $n \geq 2$, or by the Lagrange-Henrici Series Reversion Algorithm. If the period function for this problem is written as

$$
P(X, \lambda)=2 \pi+p_{1}(\lambda) X+p_{2}(\lambda) X^{2}+p_{3}(\lambda) X^{3}+p_{4}(\lambda) X^{4}+\cdots,
$$

then by the Potential Period Lemma

$$
\mathfrak{m}:=\left(p_{1}, p_{2}, p_{3}, \ldots\right)=\left(p_{2}, p_{4}, p_{6}, \ldots\right)=\left(d_{3}, d_{5}, d_{7}, \ldots\right),
$$

and consequently, by the Polynomial Potential Isochrone Theorem, $V(\mathfrak{m})=$ $\{0\}$. To simplify the notation we define

$$
q_{m}\left(\lambda_{3}, \lambda_{4}, \ldots, \lambda_{N}\right):=d_{2 m+1}\left(\lambda_{3}, \lambda_{4}, \ldots, \lambda_{N}\right)
$$

for $m \geq 1$. The Reversion Ideal Theorem can be applied to verify that the ideal $\left(d_{2}, d_{3}, d_{4}, \ldots\right)$ is radical in $\mathbf{R}\left[\lambda_{3}, \lambda_{4}, \ldots, \lambda_{N}\right]$, but the ideal $\mathfrak{m}=$ $\left(d_{3}, d_{5}, d_{7}, \ldots\right)$ is substantially more difficult to analyze. We would like to determine the smallest integer $k$ such that $m_{k}:=\left(q_{1}, q_{2}, \ldots, q_{k}\right)=\mathrm{m}$, over $\mathbf{R}\left[\lambda_{3}, \lambda_{4}, \ldots, \lambda_{N}\right]$. In the next lemma we obtain some formulas which can assist us in the analysis of this problem. 
Lemma 4.4 [Potential Reversion Coefficient Lemma]. The following relations are valid:

$$
\frac{\partial q_{m}}{\partial \lambda_{i+2}}=-\left.\frac{1}{(2 m) !} \frac{\partial^{2 m}}{\partial u^{2 m}}(1+\phi(u))^{-(2 m+3) / 2} u^{i}\right|_{u=0}
$$

and in general, if $\lambda=\left(\lambda_{3}, \lambda_{4}, \ldots, \lambda_{N}\right), \mathbf{k}=\left(k_{3}, k_{4}, k_{5}, \ldots, k_{N}\right)$, and $|\mathbf{k}|=$ $\sum_{i \geq 3} k_{i}$, then

$$
\begin{aligned}
D_{\lambda}^{\mathbf{k}} q_{m} & :=\frac{\partial^{|\mathbf{k}|} q_{m}}{\partial \lambda_{3}^{k_{3}} \partial \lambda_{4}^{k_{4}} \cdots \partial \lambda_{N}^{k_{N}}} \\
& =\left.\frac{\mu_{m,|\mathbf{k}|}}{(2 m+1) !} \frac{\partial^{2 m}}{\partial u^{2 m}}(1+\phi(u))^{-(2 m+2|\mathbf{k}|+1) / 2} u^{k_{3}+2 k_{4}+3 k_{5}+\cdots+(N-2) k_{N}}\right|_{u=0}
\end{aligned}
$$

where

$$
\mu_{m,|\mathbf{k}|}=(-1)^{|\mathbf{k}|}(2 m+1)(2 m+3) \cdots(2 m+2|\mathbf{k}|-1) .
$$

Moreover,

and for $|\mathbf{k}| \geq 2$ we have

$$
\left.\frac{\partial q_{m}}{\partial \lambda_{i+2}}\right|_{\lambda=0}=-\delta_{i, 2 m}
$$

$$
\left.D_{\lambda}^{|\mathbf{k}|} q_{m}\right|_{\lambda=\mathbf{0}}=\frac{\mu_{m,|\mathbf{k}|}}{2 m+1} \delta_{k_{3}+2 k_{4}+3 k_{5}+\cdots+(N-2) k_{N}, 2 m} .
$$

The proof is omitted since it is a straightforward induction argument.

Generally, we find that $V\left(\mathfrak{m}_{k}\right)=\{0\}$ for $k \geq N-2$, and if one computes the first row of $\hat{\mathbf{H}}(\mathbf{0})$ and the first two rows of $\mathbf{J}(\mathbf{0})^{T}$ using the formulas in the Potential Reversion Coefficient Lemma, then it is easy to see from the Exponent Two Corollary in Appendix A that the primary component of the ideal $\mathfrak{m}_{N-2}$ at 0 always has exponent greater than two. Thus the methods of Appendix A do not apply directly. Nonetheless, the ideal membership problem has a nice structure that can, at least in principal, be analyzed in any particular case. The next theorem is a useful case.

Theorem 4.3 [Even Potential Theorem]. Assume that the potential function $V(u)$ is even, i.e., let

$$
V(u)=\frac{1}{2} u^{2}+\sum_{i=1}^{n-1} \lambda_{2 i+2} u^{2 i+2} .
$$

Then $n-1$ is the smallest integer $k$ such that $\mathfrak{m}_{k}=\left(q_{1}, q_{2}, \ldots, q_{k}\right)=\mathfrak{m}$ over $\mathbf{R}\left[\lambda_{4}, \lambda_{6}, \ldots, \lambda_{2 n}\right]$. The differential equation $\ddot{u}+g(u, \lambda)=0$ corresponding to the potential function $V$ has at most $n-2$ local critical periods which bifurcate from the origin. Moreover, there are perturbations with exactly $k$ local critical periods for each $k \leq n-2$.

Proof. In this case, the function $\phi(u)$ is given by

$$
\phi(u)=2 \sum_{i=1}^{n-1} \lambda_{2 i+2} u^{2 i}
$$


We define $\lambda=\left(\lambda_{2}, \lambda_{4}, \ldots, \lambda_{2 n}\right)$ and $\mathfrak{m}_{n-1}=\left(q_{1}, q_{2}, \ldots, q_{n-1}\right)$. Then

$$
\left.\frac{\partial q_{i}}{\partial \lambda_{2 j+2}}\right|_{\lambda=0}=-\delta_{2 j, 2 i}
$$

for $i, j=1,2, \ldots, n-1$. Hence the Jacobian matrix of the transformation $\lambda \mapsto\left(q_{1}(\lambda), q_{2}(\lambda), \ldots, q_{n-1}(\lambda)\right)$ is $-\mathbf{I}_{n-1}$. It follows that $q_{i}\left(\lambda_{4}, \lambda_{6}, \ldots, \lambda_{2 n}\right)=$ $-\lambda_{2 i+2}+R_{i}\left(\lambda_{4}, \lambda_{6}, \ldots, \lambda_{2 n}\right)$, where $R_{i} \in \mathfrak{q}_{0}^{2}$. Furthermore, we observe that

$$
\left.D_{\lambda}^{\mathbf{k}} q_{i}\right|_{\lambda=0}=\mu_{m,|\mathbf{k}|} \delta_{2 k_{4}+4 k_{6}+\cdots+2 i k_{2 i+2}+\cdots+(2 n-2) k_{2 n}, 2 i},
$$

which has the value 0 whenever $k_{2 i+2}>1$. Consequently, the system of equations $q_{i}(\lambda)=0$ for $i=1,2, \ldots, n-1$ can be solved in the order $i=1,2, \ldots, n-1$ for

$$
\lambda_{2 i+2}=f_{i}\left(\lambda_{2 i+4}, \ldots, \lambda_{2 n}\right)
$$

where each $f_{i}$ vanishes when $\lambda=\mathbf{0}$. Therefore $V\left(\mathfrak{m}_{n-1}\right)=\{0\}$. Moreover, by the Exponent One Lemma in Appendix A, the ideal $\mathfrak{m}_{n-1}$ is radical. Hence $f \in \mathfrak{m}_{n-1}$ if and only if $f(0)=0$.

Since the polynomials $q_{1}, \ldots, q_{n-1}$ are independent with respect to $q_{n}$ at $\mathbf{0}$, the statement about the bifurcation of critical periods is an immediate consequence of the Isochrone Bifurcation Theorem in $\S 2$.

Example. [deg $V \leq 6$ ]. Consider the four-parameter potential function

$$
V_{6}(u):=\frac{1}{2} u^{2}+\lambda_{3} u^{3}+\lambda_{4} u^{4}+\lambda_{5} u^{5}+\lambda_{6} u^{6} \text {. }
$$

The Lagrange-Henrici Series Reversion Algorithm or the Potential Reversion Coefficient Lemma and some lengthy calculations can be used to find the polynomials $q_{1}, q_{2}, q_{3}, q_{4}, q_{5}$. If we use the change of coordinates

$$
\begin{gathered}
\lambda_{3}=\mu_{1}, \quad \lambda_{4}=\frac{5 \mu_{1}^{2}-2 \mu_{2}}{2}, \quad \lambda_{5}=\mu_{3}, \\
\lambda_{6}=\frac{14 \mu_{1} \mu_{3}-56 \mu_{1}^{4}+28 \mu_{2} \mu_{1}^{2}-2 \mu_{4}+7 \mu_{2}^{2}}{2}
\end{gathered}
$$

in the parameter space, then these polynomials are

$$
\begin{aligned}
q_{1}\left(\mu_{1}, \mu_{2}, \mu_{3}, \mu_{4}\right)= & \mu_{2}, \\
q_{2}\left(\mu_{1}, \mu_{2}, \mu_{3}, \mu_{4}\right)= & \mu_{4}, \\
q_{3}\left(\mu_{1}, \mu_{2}, \mu_{3}, \mu_{4}\right)= & \frac{9}{2} \mu_{3}^{2}-222 \mu_{1}^{3} \mu_{3}+36 \mu_{2} \mu_{1} \mu_{3}+954 \mu_{1}^{6}-621 \mu_{2} \mu_{1}^{4} \\
& +27 \mu_{4} \mu_{1}^{2}-\frac{45}{2} \mu_{2}^{2} \mu_{1}^{2}+9 \mu_{2} \mu_{4}-15 \mu_{2}^{3}, \\
q_{4}\left(\mu_{1}, \mu_{2}, \mu_{3}, \mu_{4}\right)= & -374 \mu_{1}^{2} \mu_{3}^{2}+\frac{143}{2} \mu_{2} \mu_{3}^{2}+4136 \mu_{1}^{5} \mu_{3}-5214 \mu_{2} \mu_{1}^{3} \mu_{3} \\
& +66 \mu_{4} \mu_{1} \mu_{3}+341 \mu_{2}^{2} \mu_{1} \mu_{3}-11704 \mu_{1}^{8}+25146 \mu_{2} \mu_{1}^{6} \\
& -407 \mu_{4} \mu_{1}^{4}-\frac{21021}{2} \mu_{2}^{2} \mu_{1}^{4}+561 \mu_{2} \mu_{4} \mu_{1}^{2}-\frac{1639}{2} \mu_{2}^{3} \mu_{1}^{2} \\
& +\frac{11}{2} \mu_{4}^{2}+33 \mu_{2}^{2} \mu_{4}-\frac{187}{2} \mu_{2}^{4},
\end{aligned}
$$




$$
\begin{aligned}
q_{5}\left(\mu_{1}, \mu_{2}, \mu_{3}, \mu_{4}\right)= & -130 \mu_{1} \mu_{3}^{3}+9295 \mu_{1}^{4} \mu_{3}^{2}-8190 \mu_{2} \mu_{1}^{2} \mu_{3}^{2}+\frac{195}{2} \mu_{4} \mu_{3}^{2} \\
& +\frac{975}{2} \mu_{2}^{2} \mu_{3}^{2}-85228 \mu_{1}^{7} \mu_{3}+130364 \mu_{2} \mu_{1}^{5} \mu_{3} \\
& -5980 \mu_{4} \mu_{1}^{3} \mu_{3}-56095 \mu_{2}^{2} \mu_{1}^{3} \mu_{3}+1950 \mu_{2} \mu_{4} \mu_{1} \mu_{3} \\
& -195 \mu_{2}^{3} \mu_{1} \mu_{3}+213772 \mu_{1}^{10}-463086 \mu_{2} \mu_{1}^{8} \\
& +27014 \mu_{4} \mu_{1}^{6}+349206 \mu_{2}^{2} \mu_{1}^{6}-24180 \mu_{2} \mu_{4} \mu_{1}^{4} \\
& -\frac{149435}{2} \mu_{2}^{3} \mu_{1}^{4}+585 \mu_{4}^{2} \mu_{1}^{2}+\frac{9555}{2} \mu_{2}^{2} \mu_{4} \mu_{1}^{2} \\
& -\frac{21255}{2} \mu_{2}^{4} \mu_{1}^{2}+\frac{195}{2} \mu_{2} \mu_{4}^{2}-130 \mu_{2}^{3} \mu_{4}-\frac{429}{2} \mu_{2}^{5} .
\end{aligned}
$$

The coordinate functions

$$
\left(\lambda_{3}, \lambda_{4}, \lambda_{5}, \lambda_{6}\right) \mapsto \mu_{k}, \quad k=1,2,3,4,
$$

of the inverse of our coordinate transformation are also polynomials (i.e., the coordinate transformation is a polyomorphism). Thus the ideal $\mathfrak{m}$ can be analyzed in $\mathbb{R}\left[\mu_{1}, \mu_{2}, \mu_{3}, \mu_{4}\right]$. We make a few observations which will assist us in this analysis. The Potential Reversion Coefficient Lemma implies that the polynomials $q_{m} \in \mathbb{R}\left[\lambda_{3}, \lambda_{4}, \lambda_{5}, \lambda_{6}\right]$ for $m \geq 1$ are weighted homogeneous polynomials of degree $2 m$ with weighting pattern $(1,2,3,4)$ in $\left(\lambda_{3}, \lambda_{4}, \lambda_{5}, \lambda_{6}\right)$ (see $[9, \mathrm{p}$. 195] for the terminology). The polynomials $\mu_{k}$ used to define the polyomorphism $\left(\lambda_{3}, \lambda_{4}, \lambda_{5}, \lambda_{6}\right) \mapsto \mu_{k}$ and the polynomials $\lambda_{k+2}$ for the corresponding inverse $\left(\mu_{1}, \mu_{2}, \mu_{3}, \mu_{4}\right) \mapsto \lambda_{k+2}$ are each weighted homogeneous polynomials of degree $2 k, k=1,2,3,4$, with weight pattern $(1,2,3,4)$. Hence, in the new coordinates $\left(\mu_{1}, \mu_{2}, \mu_{3}, \mu_{4}\right)$ the polynomials $q_{m}\left(\mu_{1}, \mu_{2}, \mu_{3}, \mu_{4}\right)$ for $m \geq 1$ in $\mathbb{R}\left[\mu_{1}, \mu_{2}, \mu_{3}, \mu_{4}\right]$ are weighted homogeneous polynomials of degree $2 m$, with weighting pattern $(1,2,3,4)$ in $\left(\mu_{1}, \mu_{2}, \mu_{3}, \mu_{4}\right)$. Finally we note that, by the Potential Isochrone Theorem in this section, $V(\mathfrak{m})=\{\boldsymbol{0}\}$.

If all but one of the parameters $\lambda_{i+2}, i=1,2,3,4$, are identically zero, then it is easy to show that the ideal $m$ is generated by the first polynomial in the sequence $q_{1}, q_{2}, q_{3}, \ldots$ which is not identically zero. In this case no zeros bifurcate from the origin. The ideals $\mathfrak{m}_{k}$ for all the cases where $2<\operatorname{deg} V_{6} \leq 6$ may be readily analyzed by means of the methods developed in $\S 2$. However, we confine our discussion to three main cases, and we summarize our findings as follows:

1. $\lambda_{5} \equiv 0, \lambda_{6} \equiv 0 \Rightarrow \mathfrak{m}_{2}=\left(q_{1}, q_{2}\right)=\mathfrak{m}$, and $q_{1}$ is independent with respect to $q_{2}$ at $\mathbf{0}$.

2. $\lambda_{6} \equiv 0 \Rightarrow \mathfrak{m}_{3}=\left(q_{1}, q_{2}, q_{3}\right)=\mathfrak{m}$, and $q_{1}, q_{2}$ are independent with respect to $q_{3}$ at $\mathbf{0}$.

3. No parameter $\lambda_{3}, \lambda_{4}, \lambda_{5}, \lambda_{6}$ is identically zero. In this case

$$
\mathfrak{m}_{5}=\left(q_{1}, q_{2}, q_{3}, q_{4}, q_{5}\right)=\mathfrak{m} \text {. }
$$

The polynomials, $q_{1}, q_{2}, q_{3}$ are independent with respect to $q_{4}$ at $\mathbf{0}$, but $q_{1}, q_{2}, q_{3}, q_{4}$ are not independent with respect to $q_{5}$ at $\mathbf{0}$. 
Case 1. In this case we have a two-parameter potential function

$$
V_{4}(u):=\frac{1}{2} u^{2}+\lambda_{3} u^{3}+\lambda_{4} u^{4} .
$$

It is clear that $q_{2} \notin\left(q_{1}\right)$ and $V\left(\left(q_{1}, q_{2}\right)\right)=\{0\}$. We will show that

$$
\mathfrak{m}_{2}:=\left(q_{1}, q_{2}\right)=\mathfrak{m} \text {. }
$$

If we put $\lambda_{5}=\lambda_{6} \equiv 0$ in our formulas for $q_{1}$ and $q_{2}$, we see that

$$
q_{1}\left(\lambda_{3}, \lambda_{4}\right)=-\frac{1}{2}\left(2 \lambda_{4}-5 \lambda_{3}^{2}\right), \quad q_{2}\left(\lambda_{3}, \lambda_{4}\right)=\frac{7}{8}\left(4 \lambda_{4}^{2}-36 \lambda_{4} \lambda_{3}^{2}+33 \lambda_{3}^{4}\right) .
$$

Using the change of variables, $\lambda_{3}=\mu_{1}$ and $\lambda_{4}=\frac{1}{2}\left(-2 \mu_{2}+5 \mu_{1}^{2}\right)$, discussed above, in each of the polynomials $q_{m}, m \geq 1$, we find that in this new coordinate system the ideal $\mathfrak{m}_{2}$ in $\mathbf{R}\left[\mu_{1}, \mu_{2}\right]$ can be written

$$
\mathfrak{m}_{2}=\left(\mu_{2}, \frac{7}{2}\left[\mu_{2}^{2}+4 \mu_{2} \mu_{1}^{2}-8 \mu_{1}^{4}\right]\right) \text {. }
$$

One can easily verify that $\mathfrak{m}_{2}=\left(\mu_{2}, \mu_{1}^{4}\right)$, and thus we need only prove that $q_{m}$, $m \geq 3$ does not contain a monomial, $\mu_{1}^{k}$, with $k<4$. However, the $q_{m}$ are weighted homogeneous polynomials of degree $2 m$, with weight pattern $(1,2)$ in $\left(\mu_{1}, \mu_{2}\right)$. Thus $\mu_{1}^{k}$ cannot appear in $q_{m}$, with $m \geq 3$, unless $k \geq 6$. We conclude that $q_{m} \in \mathfrak{m}_{2}$ for $m \geq 1$.

Finally we note that $q_{1}$ is independent with respect to $q_{2}$ at $\mathbf{0}$.

Case 2. Consider the three-parameter potential function

$$
V_{5}(u):=\frac{1}{2} u^{2}+\lambda_{3} u^{3}+\lambda_{4} u^{4}+\lambda_{5} u^{5} .
$$

Then since $\lambda_{6} \equiv 0$, the polyomorphic coordinate transformation

$$
\lambda_{3}=\mu_{1}, \quad \lambda_{4}=\left(5 \mu_{1}^{2}-2 \mu_{2}\right) / 2, \quad \lambda_{5}=\mu_{3},
$$

gives

$$
\begin{aligned}
q_{1}\left(\mu_{1}, \mu_{2}, \mu_{3}\right)= & \mu_{2}, \\
q_{2}\left(\mu_{1}, \mu_{2}, \mu_{3}\right)= & 7 \mu_{1} \mu_{3}-28 \mu_{1}^{4}+14 \mu_{2} \mu_{1}^{2}+\frac{7}{2} \mu_{2}^{2}, \\
q_{3}\left(\mu_{1}, \mu_{2}, \mu_{3}\right)= & \frac{9}{2} \mu_{3}^{2}-33 \mu_{1}^{3} \mu_{3}+99 \mu_{2} \mu_{1} \mu_{3}+198 \mu_{1}^{6}-495 \mu_{2} \mu_{1}^{4} \\
& +198 \mu_{2}^{2} \mu_{1}^{2}+\frac{33}{2} \mu_{2}^{3} .
\end{aligned}
$$

Now one can check that

$$
V\left(\left(q_{1}, q_{2}\right)\right) \neq\{\mathbf{0}\}=V\left(\left(q_{1}, q_{2}, q_{3}\right)\right),
$$

and thus $q_{3} \notin\left(q_{1}, q_{2}\right)$. Next we establish that

$$
\mathfrak{m}=\mathfrak{m}_{3}:=\left(q_{1}, q_{2}, q_{3}\right) .
$$

In order to do this, the following inclusion (proper) is noted:

$$
\mathfrak{q}_{3}:=\left(\mu_{2}, \mu_{1}^{7}, \mu_{1} \mu_{3}^{2}, \mu_{1}^{4} \mu_{3}, \mu_{3}^{3}\right) \subset \mathfrak{m}_{3} .
$$


It is clear that $q_{3}$ contains all monomials $\mu_{1}^{i_{1}} \mu_{2}^{i_{2}} \mu_{3}^{i_{3}}$, where $i_{1}+2 i_{2}+3 i_{3} \geq 7$ if $i_{1}+2 i_{2}>0$. Moreover, the ideal $\mathfrak{q}_{3}$ contains all monomials $\mu_{1}^{i_{1}} \mu_{2}^{i_{2}} \mu_{3}^{i_{3}}$ with $i_{3} \geq 3$. Now the $q_{m}$ are weighted homogeneous polynomials of degree $2 m$ with weight pattern $(1,2,3)$ in $\left(\mu_{1}, \mu_{2}, \mu_{3}\right)$. Hence, the only monomials $\mu_{1}^{i_{1}} \mu_{2}^{i_{2}} \mu_{3}^{i_{3}}$ which can appear in $q_{m}$ with $m \geq 4$, must satisfy $i_{1}+2 i_{2}+3 i_{3} \geq 8$. Whence if $i_{1}+2 i_{2}>0$, then the monomial is in $\mathfrak{q}_{3}$. On the other hand, if $i_{1}+2 i_{2}=0$, then $3 i_{3} \geq 8$, and thus since $i_{3}$ is a nonnegative integer, this implies $i_{3} \geq 3$. Consequently, we have $q_{m} \subset \mathfrak{q}_{3}$ for $m \geq 4$.

Case 3. In this case we have the full potential function

$$
V_{6}(u):=\frac{1}{2} u^{2}+\lambda_{3} u^{3}+\lambda_{4} u^{4}+\lambda_{5} u^{5}+\lambda_{6} u^{6} .
$$

One can establish that $q_{5} \notin\left(q_{1}, q_{2}, q_{3}, q_{4}\right)$. We claim that

$$
\mathfrak{m}=\mathfrak{m}_{5}:=\left(q_{1}, q_{2}, q_{3}, q_{4}, q_{5}\right) .
$$

It can be shown that the ideal $\mathfrak{m}_{5}$ has the following simple set of generators:

$$
\mathfrak{G}_{5}:=\left\{\mu_{2}, \mu_{4}, \mu_{1}^{10}, \mu_{1} \mu_{3}^{3}, \mu_{1}^{4} \mu_{3}^{2}, \mu_{1}^{7} \mu_{3}\right\} \text {. }
$$

In order to show that $q_{5} \notin\left(q_{1}, q_{2}, q_{3}, q_{4}\right)$ and $\mathfrak{m}_{5}=\left(\mathfrak{G}_{5}\right)$, it is useful to use an ideal membership algorithm based on the computation of a Gröbner basis for the ideal $m_{5}$. Such algorithms have been implemented in various computer algebra systems, such as MACSYMA and REDUCE, and are widely available. Using the relationship $\mathfrak{m}_{5}=\left(\mathfrak{G}_{5}\right)$ it is clear that $\mathfrak{m}_{5}$ contains every monomial of the form

$$
\mu_{1}^{i_{1}} \mu_{2}^{i_{2}} \mu_{3}^{i_{3}} \mu_{4}^{i_{4}}, \quad \text { with } i_{3}+2 i_{4}+3 i_{5}+4 i_{6} \geq 10 .
$$

Since the $q_{m}$ with $m \geq 6$ are weighted homogeneous polynomials of degree $2 m$ with weight pattern $(1,2,3,4)$ in $\mu_{1}, \mu_{2}, \mu_{3}, \mu_{4}$, it follows that $q_{m}, m \geq 6$, are formed from monomials of the form $\mu_{1}^{i_{1}} \mu_{2}^{i_{2}} \mu_{3}^{i_{3}} \mu_{4}^{i_{4}}$, where $i_{1}+2 i_{2}+3 i_{3}+4 i_{4}=$ $2 m \geq 12$. Consequently $q_{m} \in \mathfrak{m}_{5}$ for $m \geq 1$.

The polynomials $q_{1}, q_{2}, q_{3}, q_{4}$ are not independent with respect to $q_{5}$ at $\mathbf{0}$, since $V\left(\left(q_{1}, q_{2}, q_{3}, q_{4}\right)\right)=\{\mathbf{0}\}$, and $q_{5}(\mathbf{0})=0$. However, one can show that the polynomials $q_{1}, q_{2}, q_{3}$ are independent with respect to $q_{4}$ at $\mathbf{0}$.

The facts elicited in the Example and Isochrone Bifurcation Theorem in $\S 2$ imply the following result.

Theorem 4.4. The differential equation $\ddot{u}+g(u, \lambda)=0$ corresponding to the potential $V_{6}$ can have at most four critical periods bifurcating from the origin. There are perturbations which will produce $k$ critical periods for $k \leq 3$. For the potential $V_{5}$ at most two critical periods bifurcate from the origin, and there are perturbations with $k$ critical periods for $k \leq 2$. Finally, for the potential $V_{4}$ at most one critical period bifurcates from the origin, and there are perturbations with one critical period.

Remark. The calculation of $K=K(N)$, the smallest positive integer $k$ such that

$$
\mathfrak{m}_{k}:\left(q_{1}, q_{2}, \ldots, q_{k}\right)=\mathfrak{m}:=\left(q_{1}, q_{2}, q_{3}, \ldots\right)
$$


for a general polynomial potential function of the form

$$
V(u)=\frac{1}{2} u^{2}+\sum_{i=1}^{N-2} \lambda_{i+2} u^{i+2},
$$

remains a difficult unsolved problem. The Even Potential Theorem proved in this section and the foregoing example give some results. Computer algebra computations suggest the following conjecture:

If $N=\operatorname{deg} V \geq 3$, then

$$
V\left(\mathfrak{m}_{N-2}\right)=\{\boldsymbol{0}\},
$$

and

$$
K(N)=N-2+\left[\frac{N-4}{2}\right]
$$

where

$$
[x]=\left[\begin{array}{ll}
\text { largest integer } \leq x & \text { if } x>0, \\
0 & \text { otherwise. }
\end{array}\right.
$$

If $3 \leq N \leq 6$, then the conjecture is true as demonstrated in the above example. Computer algebra computations indicate the conjecture is true for $7 \leq N \leq 11$.

\section{A. ApPENDIX-Remarks on POlynomial IDEALS}

In order to use the bifurcation theorems which we have developed, we need some analytical criteria for deciding whether a given polynomial belongs to an ideal. Let $\mathbf{x}$ denote the vector whose coordinates are $x_{1}, x_{2}, \ldots, x_{n}$; then $\mathbf{K}[\mathbf{x}]=\mathbf{K}\left[x_{1}, \ldots, x_{n}\right]$ will be the polynomial domain in the indeterminates $x_{1}, \ldots, x_{n}$ with coefficients in the ground field $\mathrm{K}$ which we will always assume is either the complex numbers $\mathbb{C}$ or the real numbers $\mathbf{R}$. If $f_{1}, \ldots, f_{r} \in \mathbf{K}[\mathbf{x}]$, then $\left(f_{1}, \ldots, f_{r}\right)$ denotes the ideal generated by the polynomials $f_{1}, \ldots, f_{r}$ over $K[\mathbf{x}]$. Let $\mathfrak{m}=\left(f_{1}, \ldots, f_{r}\right)$. Then $V(\mathfrak{m})$, the variety of the ideal $\mathfrak{m}$, is defined to be the set of all points $\mathbf{z} \in \mathbb{C}^{n}$ such that

$$
f_{1}(\mathbf{z})=f_{2}(\mathbf{z})=\cdots=f_{r}(\mathbf{z})=0 .
$$

We are only interested in ideal membership tests over $\mathbf{R}[\mathbf{x}]$, however it will be convenient to prove ideal membership over $\mathbb{C}[\mathbf{x}]$, and then use the fact that this implies ideal membership over $\mathbf{R}[\mathbf{x}]$ [50, p. 161]. Max Noether's Fundamental Theorem [50, p. 163] provides a very useful tool for determining analytical conditions for a polynomial to belong to a zero-dimensional ideal. In order to make use of this result, we need to be able to calculate the exponents in the primary decomposition of a given zero-dimensional ideal $\mathfrak{m}$. If a with coordinates $a_{1}, a_{2}, \ldots, a_{n}$ is in $V(\mathfrak{m})$, and if $\mathfrak{p}_{\mathfrak{a}}=\left(x_{1}-a_{1}, x_{2}-a_{2}, \ldots, x_{n}-\right.$ $\left.a_{n}\right)$, then this entails finding the smallest integer $\sigma$ such that

$$
\mathfrak{p}_{\mathfrak{a}}^{\sigma} \subset\left(\mathfrak{m}, \mathfrak{p}_{\mathfrak{a}}^{\sigma+1}\right) .
$$


This exponent will be denoted by $\rho_{\mathrm{a}}$. Suppose the variety $V(\mathfrak{m})$ contains only a finite number of points $\mathbf{a}^{i} \in \mathbb{C}^{n}, i=1,2, \ldots, N$. We abbreviate $\mathfrak{p}_{\mathbf{a}^{i}}$ by $\mathfrak{p}_{i}$ and $\rho_{\mathbf{a}^{i}}$ by $\rho_{i}$. Then Max Noether's Fundamental Theorem says that

$$
\mathfrak{m}=\bigcap_{i=1}^{N} \mathfrak{q}_{i}, \quad \text { where } \mathfrak{q}_{i}:=\left(\mathfrak{m}, \mathfrak{p}_{i}^{\rho_{i}}\right) .
$$

It will be assumed in the results which follow that there are at least as many polynomials in the ideal $\mathfrak{m}$ as there are indeterminates, i.e., $r \geq n$.

Lemma A.1 [Exponent One Lemma]. Let the ideal $\mathfrak{m}=\left(f_{1}, \ldots, f_{r}\right)$ over $\mathbb{C}[\mathbf{x}]$ be zero-dimensional, and let $\mathbf{a} \in V(\mathfrak{m})$. In order that $\mathfrak{p}_{\mathbf{a}} \subset\left(\mathfrak{m}, \mathfrak{p}_{\mathbf{a}}^{2}\right)$ over $\mathbb{C}[\mathbf{x}]$ it is necessary and sufficient that the vectors $\nabla f_{1}(\mathbf{a}), \nabla f_{2}(\mathbf{a}), \ldots, \nabla f_{r}(\mathbf{a})$ span the space $\mathbb{C}^{n}$.

Proof. See [50, pp. 163-164].

If $f_{1}, f_{2}, \ldots, f_{r} \in \mathbb{K}[\mathbf{x}]$, then $\mathbf{J}(\mathbf{x})$ is the corresponding Jacobian matrix defined by

$$
\mathbf{J}(\mathbf{x})=\left(\begin{array}{c}
\nabla f_{1}(\mathbf{x}) \\
\nabla f_{2}(\mathbf{x}) \\
\vdots \\
\nabla f_{r}(\mathbf{x})
\end{array}\right) .
$$

We use $f_{k}^{\prime \prime}(\mathbf{x})$ to denote the $n \times n$ Hessian matrix whose entries at row $i$ and column $j$ for $i, j=1,2, \ldots, n$ are $\partial^{2} f_{k} / \partial x_{i} \partial x_{j}$.

Lemma A.2 [Exponent Two Lemma]. Let the ideal $\mathfrak{m}=\left(f_{1}, \ldots, f_{r}\right)$ over $\mathbb{C}[\mathbf{x}]$ be zero-dimensional, and let $\mathbf{a} \in V(\mathfrak{m})$. In order that $\mathfrak{p}_{\mathbf{a}}^{2} \subset\left(\mathfrak{m}, \mathfrak{p}_{\mathbf{a}}^{3}\right)$ over $\mathbb{C}[\mathbf{x}]$ it is necessary and sufficient that

$$
\mathbf{Y}^{T} \mathbf{J}(\mathbf{a})+\mathbf{J}(\mathbf{a})^{T} \mathbf{Y}+\sum_{k=1}^{r} y_{k} f_{k}^{\prime \prime}(\mathbf{x})=\mathbf{B}, \quad \mathbf{J}(\mathbf{a})^{T} \mathbf{y}=\mathbf{0},
$$

have a solution $\{\mathbf{Y}, \mathbf{y}\}$ for every complex $n \times n$ symmetric matrix $\mathbf{B}$, where $\mathbf{Y}$ is an $n \times r$ complex matrix and $\mathbf{y}=\left[y_{1}, y_{2}, \ldots, y_{n}\right]^{T}$ is an $n \times 1$ complex matrix.

Proof. We prove the result only for the case $\mathbf{a}=\mathbf{0}$, since the general case is a consequence of this special case by a simple translation of coordinates. Let $\mathbf{x}$ be written as the column vector $\left[x_{1}, x_{2}, \ldots, x_{n}\right]^{T}$. If $p_{2} \in \mathfrak{p}_{0}^{2}$, then $p_{2}$ can be written as

$$
\mathbf{x}^{T} \mathbf{B x}+R_{p_{2}}(\mathbf{x}),
$$

where $R_{p_{2}} \in \mathfrak{p}_{0}^{3}$, and $\mathbf{B}$ is an $n \times n$ complex symmetric matrix. We want to establish necessary and sufficient conditions that for each such $p_{2}$ there exist $\phi_{k} \in \mathbb{C}[\mathbf{x}], k=1,2, \ldots, r$, and $\tilde{R} \in \mathfrak{p}_{0}^{3}$ such that

$$
p_{2}=\tilde{R}+\sum_{k=1}^{r} \phi_{k} f_{k} .
$$


This last equation will be analyzed by first reducing it modulo $\mathfrak{p}_{0}^{3}$ as in [50]. To do this we note that since $f_{k}(\mathbf{0})=0$ for $k=1,2, \ldots, r$, we can write

$$
f_{k}(\mathbf{x})=\nabla f_{k}(\mathbf{0}) \mathbf{x}+\frac{1}{2} \mathbf{x}^{T} f_{k}^{\prime \prime}(\mathbf{0}) \mathbf{x},
$$

modulo $\mathfrak{p}_{0}^{3}$. Now the polynomials $\phi_{k}$ have the form

$$
\phi_{k}(\mathbf{x})=y_{k}+\sum_{j=1}^{n} y_{k j} x_{j}+\cdots
$$

for $k=1,2, \ldots, r$. Using this notation, we see that in order that $\mathfrak{p}_{0}^{2} \subset\left(\mathfrak{m}, \mathfrak{p}_{0}^{3}\right)$, it is necessary and sufficient that for each complex $n \times n$ symmetric matrix $\mathbf{B}$ there exist $\mathbf{Y}$, a complex $n \times r$ matrix, and $\mathbf{y}=\left[y_{1}, y_{2}, \ldots, y_{n}\right]^{T}$, a complex $n \times 1$ matrix, such that

$$
\frac{1}{2} \mathbf{x}^{T} \mathbf{B} \mathbf{x} \equiv \mathbf{y}^{T} \mathbf{J}(\mathbf{0}) \mathbf{x}+\mathbf{x}^{T} \mathbf{Y}^{T} \mathbf{J}(\mathbf{0}) \mathbf{x}+\mathbf{x}^{T}\left[\sum_{k=1}^{r} \frac{1}{2} y_{k} f_{k}^{\prime \prime}(\mathbf{0})\right] \mathbf{x} .
$$

If one calculates the first derivative with respect $\mathbf{x}$ on both sides of this last equation, and then evaluates the result at $\mathbf{x}=0$, one gets the second equation mentioned in the lemma. The first equation follows in a similar manner by calculating the second derivatives with respect to $\mathbf{x}$ at $\mathbf{0}$.

In order to use the Exponent Two Lemma we need to put it in a form where the solvability criterion can be more easily checked. First we introduce some additional notation. For $k=1,2, \ldots, n$ we define $\mathbf{H}_{k}(\mathbf{x})$ to be the $(n+1$ $-k) \times r$ matrix whose first row is

$$
\frac{1}{2} \frac{\partial^{2} f_{l}}{\partial x_{k} \partial x_{k}} \quad \text { for } l=1,2, \ldots, r
$$

and whose $i$ th row is

$$
\frac{\partial^{2} f_{l}}{\partial x_{k+i-1} \partial x_{k}}, \text { for } l=1,2, \ldots, r,
$$

for $i=2,3, \ldots, n+1-k$. Let the mapping $\mathbf{x} \mapsto\left(f_{1}(\mathbf{x}), f_{2}(\mathbf{x}), \ldots, f_{r}(\mathbf{x})\right)$ be denoted by $\mathbf{F}$. For $k=1,2, \ldots, n$ we define $\mathbf{J}_{k}(\mathbf{x})$ to be the $(n+1-k) \times n r$ matrix given by

$$
\mathbf{J}_{k}(\mathbf{x})=\left[\begin{array}{ccccc}
\overbrace{\mathbf{0}, \mathbf{0}, \ldots, \mathbf{0}}^{k-1}, & \mathbf{F}_{x_{k}}, & \mathbf{0}, & \ldots, & \mathbf{0} \\
\mathbf{0 , 0}, \ldots, \mathbf{0}, & \mathbf{F}_{x_{k+1}}, & \mathbf{F}_{x_{k}}, & \ldots, & \mathbf{0} \\
\vdots & \vdots & \vdots & \ddots & \vdots \\
\mathbf{0 , 0}, \ldots, \mathbf{0}, & \mathbf{F}_{x_{n}}, & \mathbf{0}, & \ldots, & \mathbf{F}_{x_{k}}
\end{array}\right]
$$


(in this display $\mathbf{0}$ is a $1 \times r$ matrix of zeros). Next we define $\hat{\mathbf{H}}(\mathbf{x})$ to be the $n(n+1) / 2 \times r$ matrix

$$
\hat{\mathbf{H}}(\mathbf{x})=\left[\begin{array}{c}
\mathbf{H}_{1}(\mathbf{x}) \\
\mathbf{H}_{2}(\mathbf{x}) \\
\vdots \\
\mathbf{H}_{n}(\mathbf{x})
\end{array}\right] .
$$

The $n(n+1) / 2 \times r n$ matrix $\hat{\mathbf{J}}(\mathbf{x})$ is defined by

$$
\hat{\mathbf{J}}(\mathbf{x})=\left[\begin{array}{c}
\mathbf{J}_{1}(\mathbf{x}) \\
\mathbf{J}_{2}(\mathbf{x}) \\
\vdots \\
\mathbf{J}_{n}(\mathbf{x})
\end{array}\right] .
$$

Corollary A.1 [Exponent Two Corollary]. Let the ideal $\mathfrak{m}=\left(f_{1}, \ldots, f_{r}\right)$ over $\mathbb{C}[\mathbf{x}]$ be zero-dimensional, and let $\mathbf{a} \in V(\mathfrak{m})$. In order that $\mathfrak{p}_{\mathbf{a}}^{2} \subset\left(\mathfrak{m}, \mathfrak{p}_{\mathbf{a}}^{3}\right)$ over $\mathbb{C}[\mathbf{x}]$ it is necessary and sufficient that for each complex $n(n+1) / 2 \times 1$ matrix b

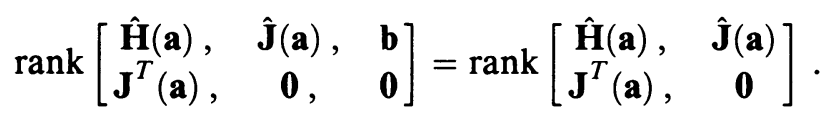

Proof. If we write out the first equation in the Exponent Two Lemma in coordinate form, we get

$$
b_{i j}=\left.\sum_{k=1}^{r}\left[\frac{\partial^{2} f_{k}}{\partial x_{i} \partial x_{j}} y_{k}+\frac{\partial f_{k}}{\partial x_{i}} y_{k j}+\frac{\partial f_{k}}{\partial x_{j}} y_{k i}\right]\right|_{\mathbf{x}=\mathbf{a}}
$$

for $i, j=1,2, \ldots, n$. Next we observe that since $\mathbf{B}$ is symmetric, there are only $n(n+1) / 2$ independent equations in the preceding list of equations. If we assemble the coefficient matrix for this list of equations in the order

$$
\begin{array}{ll}
b_{i 1}, & i=1,2, \ldots, n, \\
b_{i 2}, & i=2,3, \ldots, n, \\
\vdots & \vdots \\
b_{i, n-1}, & i=n-1, n, \\
b_{n, n}, &
\end{array}
$$

and if we let $\mathbf{b}$ denote the $n(n+1) / 2 \times 1$ matrix whose coordinates are given by the above ordering, then the solvability condition in the Exponent Two Lemma is equivalent to requiring that

$$
\left[\begin{array}{cc}
\hat{\mathbf{H}}(\mathbf{a}), & \hat{\mathbf{J}}(\mathbf{a}) \\
\mathbf{J}^{T}(\mathbf{a}), & \mathbf{0}
\end{array}\right]\left[\begin{array}{l}
\mathbf{y}_{1} \\
\mathbf{y}_{2}
\end{array}\right]=\left[\begin{array}{l}
\mathbf{b} \\
\mathbf{0}
\end{array}\right]
$$

has a solution $\left\{\mathbf{y}_{1}, \mathbf{y}_{2}\right\}$ for every complex $n(n+1) / 2 \times 1$ matrix $b$.

We are now in a position to prove the following theorem. 
Theorem A.1 [Exponent 1|2-Ideal Membership Theorem]. Let the ideal $\mathfrak{m}=$ $\left(f_{1}, \ldots, f_{r}\right)$ over $\mathbb{C}[\mathbf{x}]$ be zero-dimensional. Suppose that the points $\mathbf{a} \in V(\mathfrak{m})$ are divided into two classes, $\left(1^{0}\right)$ and $\left(2^{0}\right)$. Class $\left(1^{0}\right)$ consists of those points $\mathbf{a} \in V(\mathfrak{m})$ such that $\operatorname{rank} \mathbf{J}(\mathbf{a})=n$, and class $\left(2^{0}\right)$ is the complementary set in $V(\mathfrak{m})$. It is assumed that the rank condition of the Exponent Two Corollary is satisfied for each point a of class $\left(2^{0}\right)$. Then in order that $f \in \mathfrak{m}$, it is necessary and sufficient that the following two conditions be satisfied:

(i) $f(V(\mathfrak{m}))=\{0\}$,

(ii) $\nabla f(\mathbf{a}) \in \operatorname{span}\left\{\nabla f_{1}(\mathbf{a}), \nabla f_{2}(\mathbf{a}), \ldots, \nabla f_{r}(\mathbf{a})\right\}, \mathbf{a} \in V(\mathfrak{m})$.

Proof. If $f \in \mathfrak{m}$, then there exists $\phi_{k}, k=1,2, \ldots, r$, such that

$$
f=\sum_{k=1}^{r} \phi_{k} f_{k} \text {. }
$$

Hence condition (i), $f(\mathbf{a})=0$ for all $\mathbf{a} \in V(\mathfrak{m})$, is necessary. Moreover, since

$$
\nabla f(\mathbf{a})=\sum_{k=1}^{r} \phi_{k}(\mathbf{a}) \nabla f_{k}(\mathbf{a}), \quad \mathbf{a} \in V(\mathfrak{m}),
$$

it follows that condition (ii) must be satisfied.

It remains for us to show that if $f$ satisfies (i), (ii), then $f \in \mathfrak{m}$. If a is a point in class $\left(1^{0}\right)$, then it follows from the Exponent One Lemma that $\rho_{\mathrm{a}}=1$. We note that if $\mathbf{a} \in V(\mathfrak{m})$, then any $f \in\left(\mathfrak{m}, \mathfrak{p}_{\mathfrak{a}}^{2}\right)$ must also have the property required in (ii). This follows just as in the preceding proof of the necessity of (ii). If $\mathbf{a}$ is in class $\left(2^{0}\right)$, then we choose a vector $\mathbf{s} \in \mathbb{C}^{n}$ such that

$$
\mathbf{s} \notin \operatorname{span}\left\{\nabla f_{1}(\mathbf{a}), \nabla f_{2}(\mathbf{a}), \ldots, \nabla f_{r}(\mathbf{a})\right\} .
$$

It follows that the polynomial $\sum_{k=1}^{n} s_{k}\left(x_{k}-a_{k}\right)$ is in $\mathfrak{p}_{\mathfrak{a}}$, but it is not in $\left(\mathfrak{m}, \mathfrak{p}_{\mathbf{a}}^{2}\right)$. Thus, if $\mathrm{a}$ is a point of class $\left(2^{0}\right)$, then $\rho_{\mathrm{a}} \geq 2$. The Exponent Two Corollary then implies that $\rho_{\mathrm{a}}=2$ for all points $\mathrm{a}$ in class $\left(2^{0}\right)$.

Let $\mathfrak{q}_{\mathfrak{a}}=\left(\mathfrak{m}, \mathfrak{p}^{\rho_{\mathfrak{a}}}\right)$. Then it remains for us to show that if $f \in \mathbb{C}[\mathbf{x}]$, and $f$ satisfies (i) and (ii), then $f \in \mathfrak{q}_{\mathrm{a}}$. Noether's Theorem [50] can then be applied to infer that $f \in \mathfrak{m}$. If $\mathbf{a}$ is in class $\left(1^{0}\right)$, then $\rho_{\mathbf{a}}=1$. Hence $\mathfrak{p}_{\mathbf{a}}=\mathfrak{q}_{\mathbf{a}}$ [50] and $f \in \mathfrak{p}_{\mathbf{a}}$, since $f(\mathbf{a})=0$. Suppose $\mathbf{a}$ is in class $\left(2^{0}\right)$, and $f$ satisfies (i) and (ii) in the theorem. Then $f(\mathbf{x})=\nabla f(\mathbf{a}) \mathbf{x}+R_{f}(\mathbf{x})$, where $R_{f} \in \mathfrak{p}_{\mathbf{a}}^{2}$. Condition (ii) allows us to conclude that $f \in \mathfrak{q}_{\mathrm{a}}$.

Lemma A.3 [Two Variable Exponent Two Lemma]. Let the ideal $\mathfrak{m}=\left(f_{1}, \ldots\right.$, $\left.f_{r}\right)$ over $\mathbb{C}[x, y]$ be zero-dimensional $(r \geq 2)$, and let $\mathbf{a} \in V(\mathfrak{m})$. Then $\rho_{\mathrm{a}}=2$ if and only if one of the following two conditions is satisfied:

(i) $\mathbf{J}(\mathbf{a})=\mathbf{0}$ and $\operatorname{rank} \hat{\mathbf{H}}(\mathbf{a})=3$,

(ii) $\operatorname{rank} \mathbf{J}(\mathbf{a})=1$, and the following $5 \times 3 r$ matrix has rank 4 :

$$
\left[\begin{array}{cc}
\hat{\mathbf{H}}(\mathbf{a}), & \hat{\mathbf{J}}(\mathbf{a}) \\
\mathbf{J}^{T}(\mathbf{a}), & \mathbf{0}
\end{array}\right] \text {. }
$$


Proof. It follows from the Exponent One Lemma that $\rho_{\mathrm{a}}=2$ only when $\operatorname{rank} \mathbf{J}(\mathbf{a}) \leq 1$. The result follows from the Exponent Two Corollary by a direct calculation.

Corollary A.2 [Two Variable Ideal Membership Corollary]. Let the ideal $\mathfrak{m}=$ $\left(f_{1}, \ldots, f_{r}\right)$ over $\mathbb{C}[x, y]$ be zero-dimensional $(r \geq 2)$. Assume that for each $\mathbf{a} \in V(\mathfrak{m})$, the exponent $\rho_{\mathrm{a}}=1$ or 2 . In order that $f \in \mathfrak{m}$, it is necessary and sufficient that $f(V(\mathfrak{m}))=\{0\}$, and $\nabla f(\mathbf{a})\left\|\nabla f_{k}(\mathbf{a})\right\| \nabla f_{j}(\mathbf{a}), j, k=1,2, \ldots, r$, for each $\mathbf{a} \in V(\mathfrak{m})$ such that $\rho_{\mathbf{a}}=2$.

Proof. This follows from the Exponent One Lemma, the Two Variable Exponent Two Lemma, and the Exponent 1|2-Ideal Membership Theorem.

\section{B. APPENDIX-QUADRATIC PERIOD COEFFICIENTS}

Bautin's system $\mathbf{B}_{\lambda}$ can be written in the form

$$
\dot{x}=-y+\phi(x, y, \lambda), \quad \dot{y}=x+\psi(x, y, \lambda),
$$

where $(x, y) \mapsto \phi(x, y, \lambda)$ and $(x, y) \mapsto \psi(x, y, \lambda)$ are homogeneous polynomials of degree two, and $\lambda \mapsto \phi(x, y, \lambda)$ and $\lambda \mapsto \psi(x, y, \lambda)$ are linear. We will assume in the calculations that $\lambda$ is a general point in BV. It follows that in polar coordinates this system has the form

$$
\dot{r}=r^{2} f(\theta, \lambda), \quad \dot{\theta}=1+r g(\theta, \lambda) .
$$

Let $\theta \mapsto r(\theta, \xi, \lambda)$ denote the solution of the initial value problem

$$
\frac{d r}{d \theta}=\frac{r^{2} f(\theta, \lambda)}{1+r g(\theta, \lambda)}, \quad r(0)=\xi .
$$

It can be shown that the solution $r$ can be expanded in a convergent power series of the form

$$
r(\theta, \xi, \lambda)=\sum_{k=1}^{\infty} u_{k}(\theta, \lambda) \xi^{k},
$$

which converges for $0 \leq \theta \leq 2 \pi$ and $|\xi|$ sufficiently small. From the initial conditions we have $u_{1}(0, \lambda)=1$ and $u_{k}(0, \lambda)=0$ for $k \geq 2$. If the power series for $r(\theta, \xi, \lambda)$ is subsituted into the differential equation we obtain

$$
\left(\sum_{k=1}^{\infty} u_{k}(\theta, \lambda) \xi^{k}\right)^{2} f(\theta, \lambda)=\left(1+g(\theta, \lambda) \sum_{k=1}^{\infty} u_{k}(\theta, \lambda) \xi^{k}\right)\left(\sum_{k=1}^{\infty} u_{k}^{\prime}(\theta, \lambda) \xi^{k}\right) \text {. }
$$

This leads to the relation

$$
\begin{aligned}
f(\theta, \lambda) & \sum_{k=2}^{\infty}\left(\sum_{i=1}^{k-1} u_{i}(\theta, \lambda) u_{k-i}(\theta, \lambda)\right) \xi^{k} \\
= & u_{1}^{\prime}(\theta, \lambda) \xi+\sum_{k=2}^{\infty}\left(u_{k}^{\prime}(\theta, \lambda)+g(\theta, \lambda) \sum_{i=1}^{k-1} u_{i}^{\prime}(\theta, \lambda) u_{k-i}(\theta, \lambda)\right) \xi^{k}
\end{aligned}
$$


and from this we see that $u_{1}^{\prime}(\theta, \lambda)=0$, while for $k \geq 2$, we get the sequence of differential equations

$$
u_{k}^{\prime}(\theta, \lambda)=f(\theta, \lambda) \sum_{i=1}^{k-1} u_{i}(\theta, \lambda) u_{k-i}(\theta, \lambda)-g(\theta, \lambda) \sum_{i=1}^{k-1} u_{i}^{\prime}(\theta, \lambda) u_{k-i}(\theta, \lambda) .
$$

Since $u_{1}(0, \lambda)=1$, we conclude that $u_{1}(\theta, \lambda)=1$ for all $\theta$.

Now the period function is given by

$$
P(\xi, \lambda)=\int_{0}^{2 \pi} \frac{1}{1+r(\theta, \xi, \lambda) g(\theta, \lambda)} d \theta .
$$

The integrand is analytic for all $\theta$ and $|\xi|$ sufficiently small, and thus may be expanded in a power series of the form

$$
1+\sum_{k=1}^{\infty} a_{k}(\theta, \lambda) \xi^{k},
$$

which converges for $0 \leq \theta \leq 2 \pi$ and $|\xi|$ sufficiently small. Hence, the period function is represented by

$$
P(\xi, \lambda)=2 \pi+\sum_{k=1}^{\infty} p_{k}(\lambda) \xi^{k},
$$

where

$$
p_{k}(\lambda)=\int_{0}^{2 \pi} a_{k}(\theta, \lambda) d \theta .
$$

Finally, we compute the $a_{k}(\theta, \lambda)$ from the relation

$$
\left(1+g(\theta, \lambda) \sum_{k=1}^{\infty} u_{k}(\theta, \lambda) \xi^{k}\right)\left(\sum_{k=0}^{\infty} a_{k}(\theta, \lambda) \xi^{k}\right)=1
$$

by means of the recursive formulas: $a_{0}(\theta, \lambda) \equiv 1$, while for $k=0,1,2, \ldots$,

$$
\begin{gathered}
a_{k+1}(\theta, \lambda)=-g(\theta, \lambda) \sum_{i=0}^{k} u_{i+1}(\theta, \lambda) a_{k-i}(\theta, \lambda) . \\
\text { C. APPENDIX-NORMAL FORMS }
\end{gathered}
$$

\section{APPENDIX-NORMAL FORMS}

Given an analytic plane vector field $X$ with an equilibrium point at the origin where its linear part has eigenvalues $\pm i$, one can find an analytic transformation near the origin (cf. [40, §27]) which converts $X$ to its associated differential equation in normal form:

$$
\begin{aligned}
& \dot{x}=-y+\left(a_{3} x-b_{3} y\right)\left(x^{2}+y^{2}\right)+\left(a_{5} x-b_{5} y\right)\left(x^{2}+y^{2}\right)^{2}+\cdots, \\
& \dot{y}=x+\left(b_{3} x+a_{3} y\right)\left(x^{2}+y^{2}\right)+\left(b_{5} x+a_{5} y\right)\left(x^{2}+y^{2}\right)^{2}+\cdots .
\end{aligned}
$$


There is a center at the origin if and only if $a_{2 k+1}=0$ for all $k \geq 1$. In this case, it follows immediately that the period function for the normal form is

$$
\begin{aligned}
P(\xi) & =\frac{2 \pi}{f\left(\xi^{2}\right)}=\frac{2 \pi}{\left(1+b_{3} \xi^{2}+b_{5} \xi^{4}+b_{7} \xi^{6}+\cdots\right)} \\
& =2 \pi+p_{2} \xi^{2}+p_{4} \xi^{4}+p_{6} \xi^{6}+\cdots
\end{aligned}
$$

within the radius of convergence of the normal form. We note that the scale function $f$ and the period function $P$ have the same critical points, and by Proposition 4.2 the two ideals $\mathfrak{m}_{n f}:=\left(b_{3}, b_{5}, b_{7}, \ldots\right), \mathfrak{m}:=\left(p_{2}, p_{4}, p_{6}, \ldots\right)$ are equal.

In the case of the dehomogenized Loud system

$$
\dot{x}=-y+x y, \quad \dot{y}=x+D x^{2}+F y^{2},
$$

we obtain

$$
\begin{gathered}
b_{3}=-\left(10 D^{2}+10 D F-D+4 F^{2}-5 F+1\right) / 24 \\
b_{5}=-\left(3140 D^{4}+3880 D^{3} F+908 D^{3}+564 D^{2} F^{2}\right. \\
+552 D^{2} F+189 D^{2}-560 D F^{3}-492 D F^{2}+774 D F \\
\left.\quad-154 D-112 F^{4}-392 F^{3}+705 F^{2}-218 F+17\right) / 6912, \\
b_{7}=-\left(2619800 D^{6}+4331400 D^{5} F+1190220 D^{5}\right. \\
+1493400 D^{4} F^{2}+1049460 D^{4} F+259350 D^{4}-566920 D^{3} F^{3} \\
-558540 D^{3} F^{2}+464910 D^{3} F-34475 D^{3}-342288 D^{2} F^{4} \\
-395844 D^{2} F^{3}+385854 D^{2} F^{2}-172857 D^{2} F+25743 D^{2} \\
-56544 D F^{5}+147552 D F^{4}+242874 D F^{3}-368553 D F^{2} \\
+125220 D F-12789 D-13888 F^{6}+76272 F^{5} \\
\left.+99204 F^{4}-256699 F^{3}+110361 F^{2}-15873 F+623\right) / 3317760 .
\end{gathered}
$$

One can check that the ideal $\left(b_{3}, b_{5}, b_{7}\right)$ is equal to the ideal of period coefficients for this system as computed in the Quadratic Period Coefficient Lemma.

\section{REFERENCES}

1. A. A. Andronov, Theory of bifurcations of dynamical systems on a plane, Wiley, New York, 1973.

2. V. I. Arnold, Ordinary differential equations (R. A. Silverman, translator), MIT Press, 1978.

3. __ Geometrical methods in the theory of ordinary differential equations (J. Szücs, translator), Springer-Verlag, New York, 1983.

4. A. Baider and R. Churchill, Unique normal forms for planar vector fields, Preprint, Hunter College, 1987.

5. N. N. Bautin, On the number of limit cycles which appear with the variation of coefficients from an equilibrium position of focus or center type, Amer. Math. Soc. Transl. 100 (1954), 1-19.

6. P. Biler, On the stationary solutions of Burger's equation, Colloq. Math. 52 (1987), 305-312. 
7. T. R. Blows and N. G. Lloyd, The number of limit cycles of certain polynomial differential equations, Proc. Roy. Soc. Edinburgh Sect. A 98 (1984), 215-239.

8. N. Bourbaki, Commutative algebra, Addison-Wesley, Reading, Mass., 1969.

9. E. Brieskorn and H. Knörrer, Plane algebraic curves (J. Stillwell, translator), Birkhäuser-Verlag, Boston, Mass., 1986.

10. B. Buchberger, Gröbner bases: An algorithmic method in polynomial ideal theory, Multidimensional Systems Theory (N. K. Bose, ed.), Reidel, Boston, Mass., 1985.

11. C. Chicone, The monotonicity of the period function for planar Hamiltonian vector fields, J. Differential Equations 69 (1987), 310-321.

12. __ Geometric methods for nonlinear two point boundary value problems, J. Differential Equations (to appear).

13. C. Chicone and F. Dumortier, A quadratic system with a non monotonic period function, Proc. Amer. Math. Soc. (to appear).

14. S. N. Chow and J. K. Hale, Methods of bifurcation theory, Springer-Verlag, New York, 1982.

15. S. N. Chow and J. A. Sanders, On the number of critical points of the period, J. Differential Equations 64 (1986), 51-66.

16. S. N. Chow and D. Wang, On the monotonicity of the period function of some second order equations, Časopis Pěst. Mat. 111 (1986), 14-25.

17. R. Conti, About centers of quadratic planar systems, Universita Degli Studi di Firenze, 1986.

18. __ About centers of planar cubic systems, Universita Degli Studi di Firenze, 1986.

19. W. A. Coppel, A survey of quadratic systems, J. Differential Equations 2 (1966), 293-304.

20. J.-P. Françoise, Cycles limites études locale, Report /83/M/13, Inst. Hautes Études Sci., 1983.

21. J.-P. Françoise and C. Pugh, Keeping track of limit cycles, J. Differential Equations 65 (1986), 139-157.

22. W. Fulton, Algebraic curves, Benjamin, New York, 1969.

23. J. Guckenheimer, R. Rand, and D. Schlomink, Degenerate homoclinic cycles in perturbation of quadratic Hamiltonian systems, Preprint, 1987.

24. M. Hervé, Several complex variables, Oxford Univ. Press, 1963.

25. P. Henrici, Applied and computational complex analysis, Vol. 1, Wiley-Interscience, New York, 1974.

26. D. Knuth, The art of computer programming, Addison-Wesley, Reading, Mass., 1981.

27. W. S. Loud, Behavior of the period of solutions of certain plane autonomous systems near centers, Contributions to Differential Equations 3 (1964), 21-36.

28. V. Lunkevich and K. Sibirskii, Integrals of a general quadratic differential system in cases of a center, Differential Equations 18 (1982), 563-568.

29. A. Lyapunov, Problème général de la stabilité du mouvement, Ann. of Math. Studies, No. 17, Princeton Univ. Press, Princeton, N. J., 1949.

30. F. Murray and K. Miller, Existence theorems for ordinary differential equations, New York Univ. Press, New York, 1954.

31. L. M. Perko, On the accumulation of limit cycles, Proc. Amer. Math. Soc. 99 (1987), 515-526.

32. I. Pleshkan, $A$ new method of investigating the isochronicity of a system of two differential equations, Differential Equations 5 (1969), 796-802.

33. G. S. Petrov, Number of zeros of complete elliptic integrals, Functional Anal. Appl. 18 (1984), 73-74.

34. __ Elliptic integrals and their nonoscillation, Functional Anal. Appl. 20 (1986), 37-40.

35. T. Poston and I. Stewart, Catastrophe theory and its applications, Pitman, London, 1978.

36. R. Roussarie, private communication, 1987. 
37. F. Rothe, Periods of oscillation, nondegeneracy and specific heat of Hamiltonian systems in the plane, Proc. Internat. Conf. on Differential Equations and Math. Physics, Birmingham, Alabama, 1986.

38. G. Sansone and R. Conti, Non-linear differential equations, Macmillan, New York, 1964.

39. K. Sibirskii, On the number of limit cycles in the neighborhood of a singular point, Differential Equations 1 (1965), 36-47.

40. C. K. Siegel and J. K. Moser, Lectures on celestial mechanics, Springer-Verlag, New York, 1971.

41. R. Schaaf, A class of Hamiltonian systems with increasing periods, J. Reine Angew. Math. 363 (1985), 96-109.

42. A. Seidenberg, Elements of algebraic curves, Addison-Wesley, Reading, Mass., 1968.

43. J. Smoller and A. Wasserman, Global bifurcation of steady state solutions, J. Differential Equations 39 (1981), 269-290.

44. J. Sotomayor and R. Paterlini, Quadratic vector fields with finitely many periodic orbits, Internat. Sympos. on Dynamical Systems, I.M.P.A., Rio de Janeiro, 1983.

45. M. Urabe, Potential forces which yield periodic motions of a fixed period, J. Math. Mech. 10 (1961), 569-578.

46. - The potential force yielding a periodic motion whose period is an arbitrary continuous function of the amplitude of the velocity, Arch. Rational Mech. Anal. 11 (1962), 27-33.

47. A. N. Varchenko, Estimation of the number of zeros of an Abelian integral depending on a parameter, and limit cycles, Functional Anal. Appl. 18 (1984), 98-108.

48. W. Vasconcelos, private communication, 1987.

49. B. L. van der Waerden, Algebra, Vol. II, Ungar, New York, 1950.

50. __ Algebra, Vol. II, Ungar, New York, 1970.

51. J. Waldvogel, The period in the Lotka-Volterra system is monotonic, J. Math. Anal. Appl. 114 (1986), 178-184.

52. Yan-Qian Ye, et al. Theory of limit cycles, Transl. Math. Monographs, Vol. 66, Amer. Math. Soc., Providence, R.I., 1984.

53. O. Zariski and P. Samuel, Commutative algebra, Vol. II, Van Nostrand, Princeton, N.J., 1960.

Department of Mathematics, University of Missouri at Columbia, Columbia, Missouri 65211 